\title{
A viability analysis of sustainable by-catch fisheries under direct conservation measures
}

\author{
by \\ Stewart David Namoori Sinclair
}

\author{
A thesis \\ submitted to the Victoria University of Wellington \\ in fulfilment of the \\ requirements for the degree of \\ Master of Commerce \\ in Economics \\ Victoria University of Wellington
}

2014 



\begin{abstract}
Sustainability in fisheries is a complex concept and one that has attracted a rich history of research over time. The basic concerns of sustainability are how to reconcile ecological, economic and social requirements within the perspectives of intra - and inter-generational equity. Therefore, maintaining these requirements simultaneously is critical to achieving a perennial system and avoiding so-called "crisis" situations. It is contended that viability theory, which is a relatively new area of mathematics, rigorously captures the essence of sustainability. Using viability theory, this thesis develops two viability models based on different direct conservation measures (i.e. input and output controls) to examine the feasibility conditions under which a regulator can achieve sustainability in a fishery characterised by a "by-catch process", whereby one species is targeted and another species is incidentally caught as by-catch. The first model considers a by-catch fishery where fishing input is controlled by a regulator. The second model considers two interrelated fisheries managed using a dual quantity-price system, which is based on New Zealand's Quota Management System (QMS). For each model, the set of constraints representing the "good health" of the system are characterised using managerial priorities identified in the literature. Then, the viability kernel, which is the largest set of initial states for which there are controls that result in inter-temporal trajectories satisfying all the constraints, is approximated numerically. This is achieved by employing VIKAASA, which
\end{abstract}


is a computer application capable of generating kernel approximations. The viability kernel provides the regulator with meaningful reference values and indicators for desirable or undesirable states of the fishery, which serve as important inputs into policy decisions. This study also shows the potential for viability theory to provide policy makers with a better insight of how to integrate ecosystem considerations into the QMS. 


\section{ACKNOWLEDGEMENTS}

I wish to acknowledge and thank the following people for their contributions to the formation of this thesis.

First and foremost, my primary supervisor, Dr. Jacek Krawczyk, for his guidance, support and patience throughout this research. His words of encouragement and wisdom have inspired me to successfully complete this research in spite of the many obstacles encountered.

Dr. Philippe Lallemand, for his wealth of knowledge, which has provided me with invaluable insights into fisheries economics and particularly fisheries management in New Zealand.

Dr. John Randal, for his sound advice and encouragement throughout my academic years, and to the staff of Victoria University of Wellington and everyone who have so kindly assisted me in various ways.

I am indebted to my parents, Joseph and Rose, my sister Celine, my partner and son, Wendy and Thomas, and the rest of my family for their support, prayers and patience while I carried out this research. 
To my family 


\section{Contents}

1 Introduction 1

2 An introduction to viability theory $\quad 11$

2.1 An overview . . . . . . . . . . . . . . . . 11

2.2 A viability problem . . . . . . . . . . . . . . . 14

2.3 VIKAASA . . . . . . . . . . . . . . . . . . 19

2.3.1 Algorithms . . . . . . . . . . . . . . . . 19

2.3.2 Formulation of a VIKAASA viability problem . . . . 20

2.3 .3 Limitations . . . . . . . . . . . . . . . . . . 24

2.4 Solving a simple fishery's viability problem . . . . . . . . . . 26

2.4 .1 Basic model . . . . . . . . . . . . . . . 26

2.4.2 Presenting the "population viability" kernel . . . . . . 32

2.4.3 Presenting the "co-viability" kernel . . . . . . . . . . . 34

2.4.4 Policy advice . . . . . . . . . . . . . . 37

2.5 Summary . . . . . . . . . . . . . . . . . 38

3 An input-controlled fishery model 39

3.1 An overview . . . . . . . . . . . . . . . . . . 39

3.2 A viability model . . . . . . . . . . . . . . . . . . . . . 41 
3.2.1 The system dynamics . . . . . . . . . . . . . 4 41

3.2.2 The viability constraints . . . . . . . . . . . 45

3.2.3 The viability kernel . . . . . . . . . . . . . . . 48

3.3 The model calibration . . . . . . . . . . . . . . . . . 49

3.3.1 The base parameter set . . . . . . . . . . . . . . 49

3.3.2 The boundaries . . . . . . . . . . . . . 54

3.4 A viability analysis . . . . . . . . . . . . . . . 58

3.4.1 Presenting the viability kernels . . . . . . . . . 58

3.4.2 A comparison of the single-species and two-species viability kernels . . . . . . . . . . . . . . 6 65

3.4.3 Sensitivity tests . . . . . . . . . . . . 68

3.4.4 Policy advice . . . . . . . . . . . . . . . . 72

3.5 Summary . . . . . . . . . . . . . . . . . 75

4 An output-controlled fisheries model $\quad 77$

4.1 An overview . . . . . . . . . . . . . . . . . . 77

4.2 A viability model . . . . . . . . . . . . . . . 81

4.2.1 Outlining the Quota Management System . . . . . . 81

4.2.2 Deriving the ACE price . . . . . . . . . . . . 89

4.2.3 The system's dynamics . . . . . . . . . . . . . . 92

4.2.4 The viability constraints . . . . . . . . . . . . 95

4.2.5 The viability kernel . . . . . . . . . . . . . . . . . 98

4.3 The model calibration . . . . . . . . . . . . . . . 99

4.3.1 The base parameter set . . . . . . . . . . . . . 99

4.3.2 The boundaries . . . . . . . . . . . . . . 101

4.4 A viability analysis . . . . . . . . . . . . . . . . 102 
4.4.1 How to analyse viability kernels with more than three dimensions? . . . . . . . . . . . . . . 102

4.4.2 Population viability analysis for the base scenario . . . 104

4.4.3 Co-viability analysis for the base scenario . . . . . . . 109

4.4.4 Implications of over-catch on the co-viability kernel . . 112

4.4.5 Allowing for $\beta_{x}$ to vary over time . . . . . . . . . 118

4.4 .6 Policy advice . . . . . . . . . . . . . . . . . . . . 124

4.5 Summary . . . . . . . . . . . . . . . . . 126

5 Conclusion $\quad 129$

A Appendices for Chapter 2 135

A.1 A capturability problem . . . . . . . . . . . . . . . 135

A.2 Gordon-Schaefer model . . . . . . . . . . . . . . . . . 137

A.2.1 Resource dynamics . . . . . . . . . . . . . . 137

A.2.2 Biologically sustainable equilibrium . . . . . . . . . . 139

A.2.3 Bio-economic equilibrium . . . . . . . . . . . . . . . . 141

A.2.4 Maximum economic yield . . . . . . . . . . . . 142

B Appendix for Chapter 3 145

B.1 Parameter estimation: some brief comments . . . . . . . . . . 145

C Appendices for Chapter 4 153

C.1 Deriving the ACE price for stock $y$. . . . . . . . . 153

C.2 Supplementary Tables and Graphs . . . . . . . . . . . . . 155

Bibliography 172

$\begin{array}{ll}\text { Glossary } & 173\end{array}$ 


\section{List of Figures}

1.1 A comparison of popular management targets. . . . . . . . . 5

2.1 An illustration of the constraint set $K$ for the basic model. . . 31

2.2 Population viability kernel with viable and non-viable trajectories for the basic model. . . . . . . . . . . . . . . 33

2.3 Co-viability kernel with viable and non-viable trajectories for the basic model. . . . . . . . . . . . . . . . . . 35

2.4 Co-viability kernel with $r_{x}>q_{x} \ldots \ldots . \ldots . \ldots 37$

3.1 Population dynamics simulated for selected parameter values. 51

3.2 Population dynamics of the by-catch stock $y$ (in red) for different values of $\alpha$ and $e$. The population dynamics of stock $x$ is in blue: $r_{x}=0.4, q_{x}=0.5, L_{x}=600, L_{y}=300, x(0)=600$ and $y(0)=300 \ldots \ldots \ldots \ldots \ldots \ldots$

3.3 A comparison of the viability kernel with and without the economic constraint. . . . . . . . . . . . . 59

3.4 2D snapshots of the viability kernel for each of the three dimensions. . . . . . . . . . . . . . . . 60

3.5 Viability kernel with viable and non-viable trajectories. . . . . 62 
3.6 Time profiles associated with the viable trajectory displayed

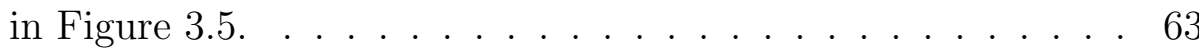

3.7 2D slices of the 3D kernel for different sizes of the by-catch biomass. . . . . . . . . . . . . . . . 64

3.8 A comparison of the two-species and single-species viability kernels for high and low initial values of the by-catch biomass. 66

3.9 Time profiles of selected variables associated with the nonviable trajectory displayed in Figure $3.8 \mathrm{~b} . \quad \ldots . . . .67$

3.10 A comparison of the viability kernels generated for $r_{y}=0.2$ and $r_{y}=0.15 \ldots \ldots \ldots \ldots \ldots \ldots \ldots$

3.11 A comparison of the viability kernels generated for $\alpha=0.2$ and $\alpha=0.3 \ldots \ldots \ldots \ldots \ldots \ldots \ldots$

3.12 A comparison of the viability kernels generated for $C=150$

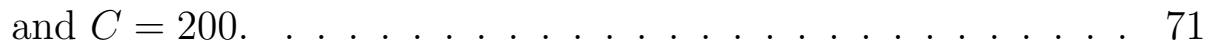

3.13 Viable paths presented in the fishing effort vs target biomass

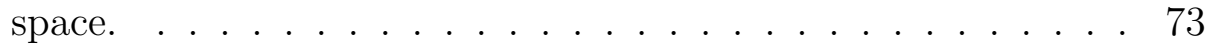

4.1 Fisheries Management Areas. Source: Ministry for Primary Industries $(2005) \ldots \ldots \ldots \ldots \ldots$

4.2 The population viability kernel for the base scenario. Panel (a) presents a $3 \mathrm{D}$ slice of the kernel through all values of $A_{y}$. Panel (b) presents a 3D slice of the kernel through all values of $A_{x} \ldots \ldots \ldots \ldots \ldots \ldots$ 
4.3 A contrast of the choice of viable TACC policies for stock $x$ under the single-species and two-species species specification, for the base scenario. Panel (a) considers only the ecological sustainability of stock $x$, and panel (b) considers the ecological sustainability of stocks $x$ and $y$ conjointly. . . . . . . . 106

4.4 A 2D projection of the population viability kernel onto the $A_{y}$ vs $A_{x}$ planes for all values of $x$ and $y$, for the base scenario. . . 107

4.5 Time profiles associated with the initial state "A" marked in Figure 4.4. The red horizontal lines represent the bounds on dynamic variables. . . . . . . . . . . . . . . . 108

4.6 A contrast of selected 3D slices of the co-viability kernel (left panels) with corresponding slices of the population viability kernel (right panels), for the base scenario. . . . . . . . . . 110

4.7 Scatter plots of the ACE prices against selected explanatory variables. The red horizontal lines represent the bounds on the ACE prices, i.e. $m_{x}^{*} \in\left(0, d_{x}\right)$ and $m_{y}^{*} \in\left(0, d_{y}\right) \ldots \ldots . .111$

4.8 Selected 3D slices of the co-viability kernel associated with over-catching $A_{x}$ (left panels) and corresponding slices of the co-viability kernel under the base scenario (right panels). . . . 113

4.9 Viable fishing profit levels associated with over-catching $A_{x}$ (panel a) and the base scenario (panel b). The horizontal axis reads the profit for agent $i$. The vertical axis reads the profit for agent $j \ldots \ldots \ldots \ldots \ldots \ldots \ldots$ 
4.10 Scatter plots of the ACE prices, $m_{x}^{*}$ and $m_{y}^{*}$ for the case where the deemed value is set at $10 \%$ of the port price for both fish stocks. The deemed values $d_{x}$ and $d_{y}$ are represented by the vertical and horizontal red lines, respectively. . . . . . . . . . 116

4.11 Viable fishing profit levels associated with the deemed value being set at $10 \%$ of the port price for different TACC levels. Panel (a) contrasts agent $i$ 's potential profits obtained for $\beta_{x}=$ 1.2 (blue area) with those obtained for $\beta_{x}=1$ (yellow area). Similarly, a contrast of agent $j$ 's profits for $\beta_{y}^{T}=1.2$ (blue area) and $\beta_{y}^{T}=1$ (yellow area) is shown in panel (b). . . . . . 117

4.12 Selected 3D slices of the augmented co-viability kernel associated with $\beta_{x}(0)=1.2$ (left panels) and $\beta_{x}(0)=1$ (right panels). . . . . . . . . . . . . . . . . . . 121

4.13 Selected 3D slices of the "augmented" co-viability kernel (left panels) and the co-viability kernel (right panels) when initially

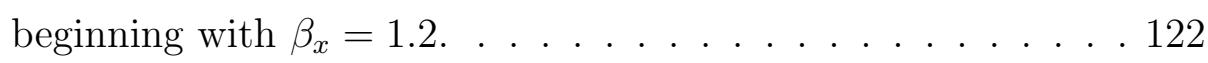

4.14 The yellow area represents the viable fishing profit domain when initially beginning with $\beta_{x}=1.2$, which remains unchanged at all times. The blue area represents the additional viable profit levels when beginning with $\beta_{x}=1.2$, but which can vary over time. The horizontal axis reads the profit for an agent in $F^{B}$. The vertical axis reads the profit for agent in $F^{T} .123$

A.1 (a) The logistic growth rate; (b) Typical solution curves . . . . 138

A.2 (a) The logistic model with constant rate of effort $e$; (b) Shaefer's yield-effort curve . . . . . . . . . . . . . . . . . 140 
A.3 (a) Bionomic equilibrium level of effort; (b) Maximisation of sustainable economic profit occurs at $e_{\mathrm{MEY}} \ldots \ldots$. . . . . 144

C.1 Monthly trends of high, low, average ACE price, annual deemed value and surveyed port prices for TAR2 (Tarakihi) between fishing years $2003-04$ to $2009-10 \ldots \ldots$. . . . . . . . 157 


\section{List of Tables}

2.1 The base parameter set for the basic model . . . . . . . . . . 30

3.1 The base parameter set for the input-controlled fishery model. 56

A.1 Management parameters for the Gordon-Schaefer model . . . . 144

C.1 Surveyed port prices for TAR2 . . . . . . . . . . . . . 155

C.2 Number of Fishing Permit Holders for TAR2 . . . . . . . . . 155

C.3 The base parameter set for the output-controlled fisheries model.156 


\section{Chapter 1}

\section{Introduction}

The scope for contribution by fisheries to global food security and economic growth is significant. In 2010, global fisheries supplied the world with about 148.5 million tonnes of fish valued at US $\$ 217.5$ billion, and supported either directly or indirectly the livelihoods of about 10-12 percent of the world's population $(F A O, 2012)$. Fish is a vital source of nutritious food that has been part of the human diet since the early stages of human evolution with the earliest definitive evidence dating back to about 1.95 million years ago (Braun et al., 2010) ${ }^{1}$. The dietary contribution of fish is significant in terms of animal proteins with about one-fifth of the world's population obtaining at least 20 percent of their animal protein intake from fish, and with some developing countries relying almost exclusively on fish (FAO, 2012). Marine fisheries also have the potential to have the lowest ecological impact of all sources of animal protein because they do not generate the waste and disease

\footnotetext{
${ }^{1}$ Fish also provides vital compounds for brain growth, which may have contributed towards the development of larger brains in the early stages of human evolution (Braun et al., 2010).
} 
problems found in both terrestrial and aquatic animal husbandry (Hauge et al., 2009).

The sad reality however is that marine resources are under extreme pressure worldwide. Recent estimates show that about three-quarters of the world's fish stocks are either fully exploited or over-exploited and that the proportion of under-exploited stocks is declining increasingly (FAO, 2012). These statistics are not surprising given that the second half of the $20^{\text {th }}$ century witnessed the remarkable growth in marine fisheries production driven by the dramatic expansion in global fishing effort, so rapid that their trend exceeded human population growth (Pauly et al., 2002) ${ }^{2}$. These are clear warning signs that fishing practices in many parts of the world are unsustainable and that there are major systematic gaps in fisheries management. As a consequence, the sustainability of fisheries has increasingly become a popular issue both nationally and internationally, and it is of particular focus in this thesis.

This thesis is also concerned particularly with the by-catch fishery management problem or simply the "by-catch problem" present in many fisheries worldwide. The spatial coexistence of marine species, imperfectly selective gear and potentially incentive-distorting managerial policies cause a significant portion of catch to diverge from the desired species (Abbott \& Wilen, 2009). As a result, a number of species are unintentionally caught as by-catch. By-catch is widely regarded as an undesirable "byproduct" of fishing. Pascoe et al. (2010) put forward several reasons why by-catch poses

\footnotetext{
${ }^{2}$ Global marine production increased from 16.8 million tonnes in 1950 and peaked at 86.4 million tonnes in 1996 (FAO, 2012). However, production has been declining in the last few years recording 77.4 million tonnes in 2010 (FAO, 2012).
} 
a major challenge for fishers, fisheries managers and conservation groups: (i) incidental catch may impose additional costs on fishers that outweigh the economic benefits from landing it, most likely resulting in the by-catch being discarded ${ }^{3}$, (ii) the mortality rate associated with discards is generally high, so where the by-catch is a commercial species there is a direct cost to the fishing industry in the form of foregone income or loss of future yield, (iii) where the by-catch is a non-commercial or protected species such as turtles, seabirds and marine mammals, discards entail non-market costs to society, (iv) discarded by-catch is often not reported, which may adversely affect the quality of stock assessments used to inform management decisions ${ }^{4}$. The significance of discarding as a wasteful and unsustainable practice is increasingly being realised given the apparent excessive exploitation of marine resources and that a significant portion of the world's population still suffers from hunger (Pascoe, 1997; FAO, 2012) $)^{5}$.

Sustainability in multi-species fisheries is a complex concept and one that has attracted a rich history of research over time (Charles, 1994). The

\footnotetext{
${ }^{3}$ These costs may include the opportunity cost of holding up storage space or quota allocation that could otherwise be used for higher valued stocks, the potential damage to high valued target species while in the nets with non-targeted stocks, and the cost associated with employing more selective fishing gear.

${ }^{4}$ For example, the underreporting of catch for a particular stock may result in its total allowable catch (TAC) being set too high. Fisheries managers try to deal with the discarding problem by placing observers on fishing vessels (Hammond \& Trenkel, 2005).

${ }^{5}$ About 7.3 million tonnes of fish suitable for human consumption are discarded every year (Kelleher, 2005). This is a substantial reduction from the 27 million tonnes proposed in Alverson et al. (1994) implying that since 1994 there have been significant efforts to improve the selectivity of fishing gear and to induce greater incentives to utilise what would otherwise be discarded.
} 
definition of sustainability and its operational content are not always well explained. However, there is a general consensus that a multi-criteria approach is required when defining sustainability (Charles, 1994). For instance, De Lara et al. (2007, p 761) define sustainability as the "ability to maintain a system within the limits of given objectives for an indefinite time". Sometimes these objectives are not compatible with each other, which therefore leads to a so-called "crisis" (or unsustainable) situation where at least one of the objectives is not met. Conflict between ecological, economic and social objectives is one of the reasons for management failure in fisheries (Hilborn, 2007; Martinet et al., 2010).

Managing the trades-offs associated with conflicting objectives also poses a challenge for fisheries management (Cheung \& Sumaila, 2008). This is illustrated in Figure 1.1, which shows popular management targets depending on the manager's goal and whether the fishery is single- or multi-species. The schematic diagram is based on the Gordon-Schaefer model commonly found in the fisheries economics literature ${ }^{6}$. The dashed lines represent the hypothetical revenue generated from harvesting a target species (in blue) and a by-catch species (in red) ${ }^{7}$. Total revenue is represented by the solid line (in black). The double hump shape of this curve is caused by the less productive by-catch species being extirpated as fishing effort increases. Total cost is made up of variable cost only and it is proportional to fishing effort. It is represented by the upward-sloping solid line (in green).

The ecological objective generally found in national legislations and inter-

\footnotetext{
${ }^{6}$ The Gordon-Schaefer model is presented in Section A.2 of Appendix A.

${ }^{7}$ Note that revenue here is the steady (or equilibrium) sustainable revenue. The unit price of fish is assumed to be constant so the corresponding sustainable yield can easily be obtained by dividing revenue by the price of fish.
} 


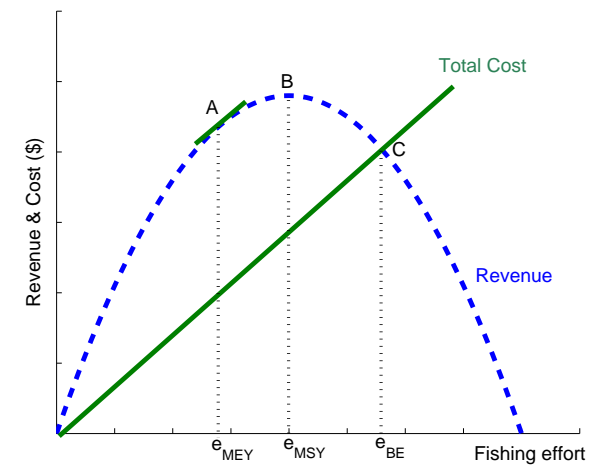

(a) Single-species fishery

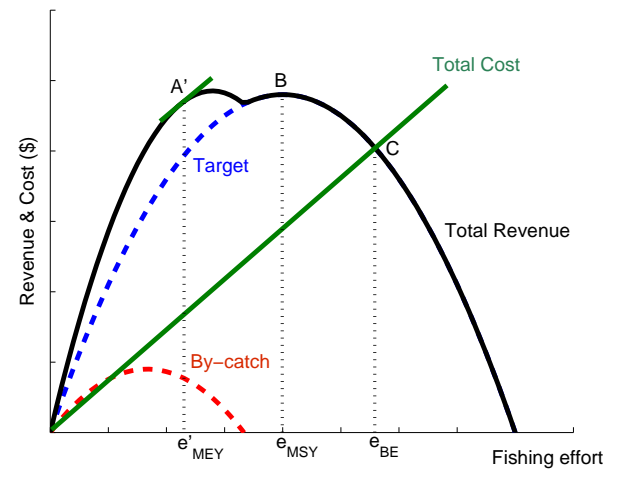

(b) Two-species fishery

Figure 1.1: A comparison of popular management targets.

national agreements is maintaining the target stock at levels that sustain the maximum level of catch or the maximum sustainable yield (MSY) (Hilborn, 2007). This requires that the fishery be maintained at B in Figure 1.1 either by enforcing a limit on fishing effort equal to $e_{\mathrm{MSY}}$ or setting a catch limit at the appropriate level. However, from an economic perspective, maintaining the fishery at A is desired because it maximises economic rent. Such a target is commonly referred to as the maximum economic yield (MEY) and generally results in foregoing higher production for more profit (Kompas et al., $2011)^{8}$. If fishing effort is an indicator of employment in the fishery, then social advocates for employment would prefer that the fishery be maintained at the "bionomic equilibrium" (Gordon, 1954), i.e. at C, which supports the highest level of fishing effort $e_{\mathrm{BE}}$ and therefore the highest level of em-

\footnotetext{
${ }^{8} \mathrm{MEY}$ is also more conservative in the sense that stocks are larger than stocks at MSY, so the fishery is more resilient to larger negative shocks to the fish population (Kompas et al., 2011).
} 
ployment. For an analytical derivation of the management parameters $e_{\mathrm{MSY}}$, $e_{\mathrm{MEY}}$ and $e_{\mathrm{BE}}$, and the corresponding biomass and yield levels, see Section A.2.

Furthermore, the protection of stocks taken as by-catch is vital as alluded to earlier. It is therefore of particular interest to consider the policy implications of setting targets in a fishery whereby multiple species with different productivity are caught jointly. For instance, setting a management target appropriate for the target stock, e.g. at B or $\mathrm{C}$ in Figure 1.1b would be utterly devastating for the less productive by-catch species as it would be driven to extinction.

Managing the above trade-off becomes more difficult when considering the bio-economic dynamics and uncertainties inherent in fishery systems. For instance, natural variability in stocks, uncertainty in the price of fish or the precise cost of fishing imply that the "Total Revenue" and "Total Cost" curves in Figure 1.1 shift up and down in a hard-to-predict fashion, making it all but impossible to determine management targets such as MSY and MEY (Kompas et al., 2011).

Traditionally, multi-objective problems such as those presented above are solved explicitly by defining a criterion that encompasses all of the objectives to be optimised. This however requires assigning weights to all of the objectives in order to materialise the trade-offs between conflicting objectives. According to Martinet et al. (2010), determining these weights can be difficult because they reflect the relative importance of different objectives, which depends on normative choices. The choice of weights and therefore the optimal solution can be influenced by the relative bargaining power of stakeholders. 
Alternatively, it may be easier and more practical to represent the objectives by thresholds or constraints that materialise the "good health" or sustainability of the fishery (Martinet et al., 2010). The regulator's problem then is to avert a crisis by finding a way or strategy that maintains the fishery within the realms of safety defined as such by these constraints. Sustainability appears then to be more of satisficing problem in the sense prescribed in Simon (1955). That is, there are bounds on human rationality and as a consequence we seek to attain some sufficient level of a goal variable rather than the constrained optimum. In fact, experience shows that humans may know enough to model the conditions under which systems are likely to persist as sources of goods and services, rather than the theoretical "optimum" conditions (Cury et al., 2005).

This thesis employs viability theory (Aubin, 1991), which has been explicitly developed for the purpose of analysing dynamic systems that face constraints, making it particularly well suited for modelling and solving sustainability problems. The viability approach allows for the characterisation of initial conditions from which there exists a control strategy that ensures that the system evolves without ever violating any of the constraints. The largest set of such initial conditions is called the viability kernel. Solving a viability problem thus requires the computation of the viability kernel, which can be a complex task depending on the dimensionality of the problem at hand. For this, in this thesis, the viability kernel is computed numerically using VIKAASA ${ }^{9}$, a computer application dedicated to solving and analysing

\footnotetext{
${ }^{9}$ Viability Kernel Approximation Analysis and Simulation Application. The Sanskrit word vikaasa (विकास) means "progress" or "development". The developers of VIKAASA (Jacek B. Krawczyk and Alastair S. Pharo) believe that their application represents vikaasa
} 
viability problems.

Two specific fisheries management regimes are considered. The first considers a fishery managed by an input control, which is defined as a restriction put on the intensity of use of gear that is used to catch fish (Pope, 2002). For instance, restrictions on the number or size of fishing vessels, the length of the fishing season, or the type of fishing gear. The other considers a fishery managed by an output control, which is defined as a limit placed upon the weight or the number of fish that is withdrawn from a fishery in a period of time (Pope, 2002). For instance, annual total allowable catches or daily bag limits used in many recreational fisheries.

Here is how this thesis is organised. Chapter 2 discusses viability theory further and formalises the theoretical framework underlining a viability problem. It also formally introduces VIKAASA, which is used to solve and analyse the viability models presented in this thesis. The goodness of the VIKAASA kernel approximation is verified by reproducing numerically the viability kernel for a calibrated single-species fishery problem that has been solved analytically in Béné et al. (2001). Chapter 3 applies the viability approach to solve a dynamic bio-economic model of a two-species fishery characterised by joint production and fixed production capacity. Here, management of the fishery is based on an input control or more specifically a single command variable that controls variation in fishing effort. Chapter 4 casts the viability problem in a dual "quantity-price" managed fishery based on New Zealand's integrated quota management and deemed value systems. This provides a richer platform for policy making, particularly in the context

in the process of understanding and application building in viability theory (Krawczyk \& Pharo, 2011). VIKAASA runs in MATLAB ${ }^{\circledR}$ and GNU Octave. 
of fisheries management in New Zealand. In essence, management here is directed at limiting the amount of fish that is commercially caught (i.e. output control). The thesis is wrapped up in Chapter 5 by presenting the conclusions and limits drawn from this research. Some discussion is also made as to where the research might be extended. 


\section{Chapter 2}

\section{An introduction to viability theory}

\subsection{An overview}

Viability theory, a relatively young area of mathematics, was first developed by French mathematician Jean-Pierre Aubin in the late 1970s (Aubin et al., 2011). The theory was inspired by the remarks attributed to the Greek philosopher Democritus by Jacques Monod that "[e]verything that exists in the Universe is due to Chance and Necessity" (Aubin, 1991, p ix). Dynamic systems are considered to evolve under contingent, stochastic or tychastic ${ }^{1}$ uncertainty such that their evolutions are constrained to adapt to the systems' environment. This is particularly true in economics where agents have to adapt to scarcity constraints, balances between supply and demand, and many other constraints. This is equally true in biology where the human

\footnotetext{
${ }^{1}$ The term "tychastic" originates from "tyches" meaning "chance" in classical Greek.
} 
body for instance has to maintain a constant internal environment (e.g. body temperature) in spite of environmental changes, a biological principle known as "homeostasis".

The basic problem (referred henceforth as viability problem) that viability theory attempts to solve is whether, for a given initial state, a control strategy exists such that the system's evolution remains confined to pre-determined constraints or corridors, beyond which its perennial state cannot be maintained. The set of all initial states from which it is possible to remain within these corridors dynamically is called the viability kernel, and hence becomes a useful tool for analysing viability problems.

Suppose that these corridors are constrained by normative measures representing knowledge of the conditions required to achieve sustainability or avert catastrophic developments. Viability theory then provides the right analytical framework to address so-called "sustainability problems" whose "solution" consists of finding a way to avert catastrophic outcomes by maintaining the system within the realms of safety or acceptability (Krawczyk \& Pharo, 2013).

The main attraction of viability theory, particularly for the purpose of this thesis, therefore lies in the results it provides. Some of these results are noted here. Firstly, it formally defines the boundary of the viability kernel, which is a useful tool for analysing sustainability problems. Secondly, viability theory is not concerned with determining theoretical optimum states, but the feasibility conditions that allow for multiple objectives to be fulfilled simultaneously at any time. The viability strategy or solution is therefore satisficing (i.e. good enough) in sense prescribed in Simon $(1955)^{2}$. Given

\footnotetext{
${ }^{2}$ This thesis shares the contention that economic agents display bounded rationality
} 
this, economic results obtained through viability theory might be closer to what economic agents are really after. Thirdly, viability theory provides a dynamic and multi-criteria framework that is well equipped to reconcile potentially conflicting management objectives (e.g. ecological, economic and social objectives). Lastly, viability theory accommodates inter-generational equity by assigning equal weight to every time period. It therefore provides an interesting analytical framework to deal with sustainable development, which places an emphasis on meeting "the needs of the present without compromising the ability of future generations to meet their own needs" (WCED, 1987, p 43) ${ }^{3}$.

Viability theory has been successfully applied to a variety of problems across many disciplines. It has been notably applied to the sustainable management of natural resources and especially fisheries, see Béné et al. (2001); Eisenack et al. (2006); De Lara et al. (2007); Martinet \& Doyen (2007); Chapel et al. (2008); De Lara \& Martinet (2009); Péreau et al. (2012); Doyen \& Péreau (2012) among others. For a viability analysis of fisheries recovery programs, see Martinet et al. (2007, 2010). For a viability perspective on the ecosystem approach to fisheries, see Bonneuil (2003); Mullon et al. (2004); Cury et al. (2005); De Lara et al. (2012); Doyen et al. (2012); Cissé et al. (2013). These papers model multi-species fisheries and species interactions. However, they do not consider the by-catch problem per se, which this thesis aims to address.

Other applications can also be found in the areas of aerodynamics ${ }^{4}$ (Oishi et al., 2006), finance (Aubin et al., 2005), macro-economics (Krawczyk \& and therefore are only after satisficing outcomes rather optimal outcomes.

${ }^{3}$ World Commission on Environment and Development.

${ }^{4}$ For a brief example, see Section A.1 of the Appendix to this chapter. 
Kim, 2013, 2009; Bonneuil \& Saint-Pierre, 2008), micro-economics (Krawczyk et al., 2012; Krawczyk \& Serea, 2011), and sociology (Bonneuil, 2000). Aubin et al. (2011) provides a good illustration of other applications, including engineering (i.e. robotics) and environmental (i.e. climate research) applications.

This chapter is organised as follows. Section 2.2 presents a general formulation of a viability problem and defines basic concepts. Section 2.3 introduces VIKAASA, a numerical application used to generate the viability kernels for the viability problems considered in this thesis. This section also includes some discussion on how to formulate a viability problem in VIKAASA, what are the key ingredients required for VIKAASA to work, and how the algorithm(s) underlining VIKAASA works. The limitations of VIKAASA are also highlighted. Section 2.4 verifies the goodness of the VIKAASA kernel approximation by reproducing numerically the viability kernel for a calibrated single-species fishery problem that has been solved analytically in Béné et al. (2001). This also serves as a good "warm up" before analysing more complex viability models in subsequent chapters. Section 2.5 wraps up this chapter by providing some concluding remarks.

\subsection{A viability problem}

Following standard practice in mathematical papers about viability theory, the dynamics of a non-deterministic system are represented as a differential inclusion $^{5}$,

$$
\dot{x}(t) \in F(x(t)),
$$

\footnotetext{
${ }^{5}$ A differential inclusion is the mathematical translation of "chance" (Aubin et al., 2011). It can be thought of as a set-valued equivalent of a differential equation.
} 
which states that at $x(t)$ the change in the system's state, i.e. its velocity, will be a member of $F(x(t))$, where $F$ is a function that takes the system's state and returns a set of possible velocities. Exactly which velocity will eventuate is subject to uncertainty, which may come from either "disturbance" and "perturbation" of various kinds, or from modeling errors due to the impossibility of a comprehensive description of the system dynamics and/or parameters (Aubin, 1990; Krawczyk \& Pharo, 2013). Regulation is also considered a potential source of uncertainty given that commitment by a regulator to a particular "closed-loop" control policy is not assumed in viability theory (Krawczyk \& Pharo, 2013).

Suppose that the dynamics of the system are related to certain "controls", which are restricted by state-dependent constraints. The multi-valued map $F$ then has the form $F(x) \equiv\{f(x), u) ; u \in U(x)\}$. In this case, the dynamic system (2.1) can be described as

$$
\begin{aligned}
& \dot{x}(t)=f(x(t), u(t)) \\
& u(t) \in U(x(t)),
\end{aligned}
$$

where (2.2) is a standard parameterised differential equation and (2.3) states that the control choice $u(\cdot)$ must come from a potentially state-dependent set, $U(x(t)) \subset \mathbb{R}^{m}$. So for a given $x(t)$, there is an array of possible controls to choose from in $U(x(t))$ and hence have a set of points in the state-space, which can be reached at time $t+\gamma$ (where $\gamma>0$ is small). For example, in a fishery-management problem, the fish biomass "tomorrow" will be in a cone determined by the apex at the present state and rays corresponding to different fishing strategies. 
Furthermore, let $K$ represent some closed set of (viability) constraints that state $x(t)$ must satisfy for all $t^{6}$,

$$
x(t) \in K, \quad \forall t \in \Theta .
$$

Given a set-valued map $F: K \rightsquigarrow X$, it is said that $x(0) \in K \subset X$ is viable in $K$ under $F$ if there exists at least one solution to the following system:

$$
\forall t \in \Theta\left\{\begin{array}{l}
x(t) \in K \\
\dot{x}(t) \in F(x(t))
\end{array}\right.
$$

that starts at $x(0)$ and remains in $K$ indefinitely $^{7}$.

The viability problem is thus concerned with determining those members of the control set $U(x(t))$ for which the system's evolutions are viable in $K$. Given such a problem, viability theory attempts to establish the existence of the viability domain, $D \subseteq K$, which indicates a viable area for which there are sufficient controls that maintain the system in $K$ from any point in $D^{8}$. That is, for every element $x(0) \in D$, there exists a function (or feed-back rule) $g: \mathbb{R}^{n} \mapsto \mathbb{R}^{m}$ that takes each element, $k$, of the constraint set $K \subset \mathbb{R}^{n}$

\footnotetext{
${ }^{6}$ This requirement (2.4) provides the mathematical translation of "necessity" (Aubin et al., 2011).

${ }^{7}$ Viability is normally defined in terms of an infinite time horizon, i.e. $\Theta \equiv[0, \infty]$, which is the case for the viability problems considered in this thesis. However, it is also possible to define $\Theta \equiv[0, T], T \in \mathbb{R}_{+}$, and talk about finite-time viability or a capturability problem, which is discussed briefly in Section A.1 in the Appendix to this chapter.

${ }^{8}$ The viability theorem underlining the existence of the viability domain requires establishing a relationship between any closed set of points $D$ viable under $F$, and the concept of the contingent cone to $D$ at $x$, which defines the trajectories that point "inside" of $D$, starting from $x$. See e.g. Krawczyk et al. (2013, p. 375) and the relevant citations therein for a more formal discussion.
} 
and returns a control policy $u$ such that

$$
\left\{\begin{array}{l}
g(k) \in U(x) \\
x(t) \in K \quad \forall t \in \Theta
\end{array}\right.
$$

where $x(t)$ is a solution to $(2.5)$.

The main theorem of viability theory asserts that the problem's viability kernel, $\mathcal{V}_{F}(K) \subseteq K$ is the largest possible viability domain, giving all initial conditions in $K$, for which a set of controls in $U(x(t))$ exists to prevent the system from exiting $K$ over $t \in \Theta$. The viability kernel of $K$ is formally defined below.

Definition 2.2.1. The viability kernel of the constraint set $K$ for the dynamics $F$ is the largest set of initial conditions $x(0) \in K$, denoted by $\mathcal{V}_{F}(K)$, and defined as follows:

$$
\mathcal{V}_{F}(K) \equiv\left\{\begin{array}{l|l}
x(0) \in K: & \begin{array}{l}
\exists u(\cdot) \text { such that the solution } x(\cdot) \text { of }(2.1) \\
\text { starting from } x(0) \text { remains in } K, \forall t \in \Theta
\end{array}
\end{array}\right\} .
$$

Given the control system described by (2.2) and (2.3), the viability constraint (2.4), and the time horizon $\Theta$, the associated viability problem thus consists of establishing the existence of the viability kernel. When the kernel is non-empty, i.e. $\mathcal{V}_{F}(K) \neq \emptyset$, the viability problem is said to poses a solution. The viability kernel is an important policy tool, in that it can be used to formulate control rules that maintain the system's sustainability.

As a summary to this section, the key elements of a viability problem are summarised and presented in Box 1 below. 


\section{Box 1. General specifications for a viability problem}

1. A continuum of time values, $\Theta \equiv[0, T]$, where $T$ can be finite or infinite.

2. A vector of real-valued state variables, $x(t) \equiv\left[x_{1}(t), x_{2}(t), \ldots x_{n}(t)\right]^{\prime} \in$ $\mathbb{R}^{n}, \forall t \in \Theta$ that together represent the dynamic system.

3. A constraint set, $K \subset \mathbb{R}^{n}$, which is a closed set representing some normative constraints imposed on these state variables.

4. A vector of real-valued controls, $u(t) \equiv\left[u_{1}(t), u_{2}(t), \ldots u_{m}(t)\right]^{\prime} \in$ $\mathbb{R}^{m}, \forall t \in \Theta$, that represent the set of feasible controls available to the decision maker.

5. A set of normative restrictions imposed on the controls, such that $u(t) \in$ $U(x(t)) . U: \mathbb{R}^{n} \mapsto \mathbb{R}^{m}$ is a set-valued function, which gives the set of control vectors available at each state.

6. The system dynamics can be represented as a set of real-valued firstorder differential inclusions,

$$
\dot{x}(t) \in F(x) \equiv\{f(x, u), u(t) \in U(x(t))\} .
$$

The next section introduces an application developed to generate approximate viability kernels using numerical methods, which spares the user of the complexity of solving for the viability kernel analytically. 


\subsection{VIKAASA: A numerical method for the determination of viability kernels}

Solving a viability problem, such as that outlined in Box 1, requires computation of the problem's viability kernel. Computation of the viability kernel can be a very complex exercise, and the level of difficulty increases with the dimensionality of the problem. Although an analytical characterisation of the viability kernel is possible, for instance in Béné et al. (2001), in most cases numerical methods have been the preferred means of overcoming the complexity of computing viability kernels. One such application that makes use of numerical methods is VIKAASA, which is used to compute the kernel approximations of viability problems considered in this thesis.

There are other kernel computation algorithms, including those proposed in Frankowska \& Quincampoix (1990); Saint-Pierre (1994); Deffuant et al. (2007). Brief comments on these algorithms are presented in Krawczyk \& Pharo (2013). For a reference list of more algorithms, see Krawczyk et al. (2013).

\subsubsection{Algorithms}

VIKAASA attempts to establish the viability kernel $\mathcal{V}_{F}(K)$ by looking for solutions to (2.5). Gaitsgory \& Quincampoix (2009) provide the base for how to do this. In broad terms, if an optimal control problem can be solved from $x(0) \in K$ and the optimal solution $x(\cdot)$ starting from $x(0)$ satisfies $x(t) \in K, \forall t$, then $x(0)$ is viable.

The are two kernel approximation algorithms that are currently imple- 
mented in VIKAASA. The "rejection" algorithm is based on the above method. It approximates the viability kernel by the locus of state-space positions for which solutions to an auxiliary cost-minimising optimal control problem can be found over a grid of state-space points $x^{\delta} \in K^{\delta} \subset K$, where $K^{\delta}$ is a suitably discretised $K$. The other ("inclusion") algorithm in contrast solves a truncated optimal stabilisation (regulation) problem. That is, for each point in $K^{\delta}$, the algorithm accepts a point as viable if the system's dynamics can be stabilised without leaving $K^{\delta}$ in finite time.

The rejection algorithm has only just been implemented in VIKAASA, so it is not considered in this thesis. Nonetheless, for a detailed discussion of the rejection algorithm and its performance relative to the inclusion algorithm, see Krawczyk et al. (2013). The numerical results presented in this thesis have thus been generated using the inclusion algorithm exclusively. The inclusion algorithm (referred henceforth simply as the algorithm) is extensively discussed in Krawczyk \& Pharo (2013) and Krawczyk \& Pharo (2011). For convenience, in what follows, an explanation of how the algorithm works as well as the key inputs required for the algorithm to work are presented.

\subsubsection{Formulation of a VIKAASA viability problem}

For a viability problem to be compatible with VIKAASA, the specifications outlined in Box 2 must be satisfied. Note that a comparison between Boxes 1 and 2 shows that only a limited class of viability problems can be analysed with VIKAASA. 


\section{Box 2. Specifications for a VIKAASA viability problem}

1. The continuum of time values must be infinite, i.e. $\Theta \equiv[0, \infty)^{9}$.

2. There are no technical limits to how many state variables that can be specified. However, in order for visualisation of the resulting viability kernel to be possible, there must be at least two state variables.

3. Although any constraint set definable as a subset of a "rectangular" set (hyper-rectangle) can be specified, the underlying algorithm requires that the containing hyper-rectangle be explicitly given as a set of upperand lower-bounds. That is, $K \subseteq\left[\underline{x}_{1}, \bar{x}_{1}\right] \times\left[\underline{x}_{2}, \bar{x}_{2}\right] \times \cdots \times\left[\underline{x}_{n}, \bar{x}_{n}\right] \equiv \hat{K}$, where $\underline{x}_{i}$ is the $i^{\text {th }}$ variable's lower bound, and $\bar{x}_{i}$ is its upper bound.

4. Multiple control variables can be specified, i.e. $m \geq 1$.

5. The control set, $U(x)$ must be the same for all values of $x$, and must be symmetrical about zero. That is, given that $u$ is a scalar, $U=$ $[-c, c], \forall x$, where $c \in \mathbb{R}_{+}$.

6. VIKAASA can only work with deterministic autonomous system's dynamics. For any given point in the state-space, and any given control choice, there can only be one possible trajectory. That is, this application cannot model stochastic processes. It should also be noted that the kernel approximation algorithm may not perform well with highly non-linear differential inclusions, due to the simple numerical methods employed to solve them. 
In addition to the standard formulation specified in Box 2, the inclusion algorithm also requires the following parameters in order to approximate viability kernels:

1. A discretisation, $\delta=\left[\delta_{1}, \delta_{2}, \ldots, \delta_{n}\right]^{\prime} \in \mathbb{R}^{n}$, which determines the finite subset of $K$ to be examined by the algorithm.

2. A stopping tolerance, $\epsilon \in \mathbb{R}_{+}$is used as the criterion for "near-steadiness" of the system.

3. A step size, $h \in \mathbb{R}_{+}$is needed by the approximation algorithm in order to compute the system's trajectories using the Euler method.

4. A control algorithm, $u^{*}: \mathbb{R}^{n} \rightarrow U$, which is a stationary feedback rule, responsible for slowing the system's velocity until its norm falls below the stopping tolerance, $\sqrt{n} \cdot \epsilon$.

The inclusion algorithm begins by dividing the constraint space $K$ along its $n$ vertices into $\delta$ evenly spaced points, starting at $\underline{x}_{i}$ and finishing at $\bar{x}_{i}, \forall i=1, \ldots, n$, and then combining these points to obtain a discretised version of the constraint set, $K^{\delta}$, which contains $\delta^{n}$ points. For each point $x(0) \in K^{\delta}$, the algorithm considers whether a first-order Euler approximation of the system can be brought to a "near-steady" state within some finite time without violating the constraints. Near-steadiness is achieved when the Euclidean norm of the system's velocity,

$$
\|f(x, u)\|=\sqrt{\dot{x}_{1}(x, u)^{2}+\dot{x}_{2}(x, u)^{2}+\cdots+\dot{x}_{n}(x, u)^{2}}
$$

\footnotetext{
${ }^{9}$ Capturability problems such as those specified in Section A.1 cannot be solved in VIKAASA.
} 
is less than the norm of a movement of $\epsilon$ in every direction, $\sqrt{n} \cdot \epsilon$.

VIKAASA offers a number of cost-minimising rules (control algorithms) for use with the inclusion algorithm ${ }^{10}$. The most sophisticated of these and the one employed to generate the numerical results of this thesis, uses MATLAB's fmincon nonlinear optimisation routine to choose the control $u \in U$ at each time realisation that minimises the norm of the system's velocity one Euler time-step into the future ${ }^{11}$, so that $u^{*}(x)=\arg \min _{u}^{G}\{\| f(x+h$. $f(x, 0), u)||\}^{12}$.

The effectiveness of the control $u^{*}$ in slowing the system is contingent on the particular point in $K^{\delta}$ under consideration. If the system can be consistently decelerated from $x(0)$, and if this can be done fast enough, then the algorithm will be able to bring the system's velocity below the predetermined threshold, in which case $x(0)$ is considered viable and it is included in the approximate viability kernel $\mathcal{V}_{F}^{\delta}(K)$. Otherwise, the control will not be effective in slowing the system, in which case either the system will leave the constraint set, or it will loop (or "orbit") infinitely ${ }^{13}$, so the algorithm

\footnotetext{
${ }^{10}$ VIKAASA also allows for the possibility of writing one's own control algorithm. Instead of minimising the Euclidean norm of the system's velocity, the user may specify some other cost function to be minimised. The VIKAASA manual (Krawczyk \& Pharo, 2011) provides further details.

${ }^{11}$ More forward-looking steps will identify more viable points, but at the cost of increased computational time.

${ }^{12}$ The symbol $\min ^{G}$ refers to the numerical method of function minimisation employed, for this thesis it is fmincon.

${ }^{13}$ As the algorithm is not interested in the content of $f(\cdot, \cdot)$, but simply attempts to solve (2.5), it is technically undecidable as to whether the algorithm will ever finish. For this reason, the algorithm gives up after some maximum number of loops, i.e. $t=46,000$. Consequently, some viable points will be missed by the algorithm if the evolutions starting
} 
cannot establish that $x(0)$ is viable and consequently it is excluded.

Thus, from each point in $K^{\delta}$, the numerical simulation routine iterates the system for as many steps as it takes either to violate one of the constraints or for the Euclidean norm to fall below the predetermined criterion ${ }^{14}$.

\subsubsection{Limitations}

However, it should be noted that there are important limitations in the inclusion algorithm. Briefly,

- The algorithm can only work with viability problems that are formulated according to the specifications provided in Box 2.

- The algorithm suffers from the "dimensionality curse", which comes from the need to discretise the constraint space in order to compute how the dynamical system behaves locally everywhere in the statespace. The computational time for a kernel approximation increases exponentially with the number of dimensions, leading to some very long waiting times for "kernel runs" to complete ${ }^{15}$.

- The algorithm depends on the user's choices of the parameters: $\delta, \epsilon$, $h$ and $u^{*}$, which affect the "goodness" of the approximation. For instance, the algorithm can falsely identify points as viable when they are

at these points are large orbits.

${ }^{14}$ This is of course subject to the requirement that the number of iterations does not exceed 46,000, see footnote 13 .

${ }^{15}$ For the four dimensional problem presented in Chapter 4, computation of its kernel took 16 hours on a PC with an Intel ${ }^{\circledR}$ Core $^{\text {TM }}$ (Quad) i7-3630QM 2.4GHz 6MB Processor and 8GB DDR3 RAM, using all four processors in parallel. 
not (i.e. false positive), if the stopping tolerance is too "generous", in which case verification of any results with a tighter grid and/or tighter tolerance is something that should perhaps be considered. Conversely, the algorithm may identify points as non-viable when they are actually viable. This can be observed for some highly non-linear dynamical systems due to the simple numerical methods employed to solve them. Increasing the number of forward-looking steps $s$ improves the effectiveness of the control algorithm $u^{*}$ in determining the viability (or non-viability) of state-space points, and thus addressing these "false negatives". However, Krawczyk \& Pharo (2011) find that $s>2$ is not practical because of the extremely long waiting times involved in computing the control.

For most viability problems, the "true" kernel $\mathcal{V}_{F}(K)$ is unknown unless it can be determined analytically, which makes verifying that the approximated kernel $\mathcal{V}_{F}^{\delta}(K)$ closely resembles $\mathcal{V}_{F}(K)$ a rather impossible task. To satisfy VIKAASA users of the "goodness" of the VIKAASA kernel approximation and therefore the quality of the numerical results presented in the subsequent chapters of this thesis, the next section presents the viability kernel generated by VIKAASA for a calibrated fishery problem, whose kernel has been established analytically in Béné et al. (2001). 


\subsection{Solving a simple fishery's viability prob- lem}

Here, a bio-economic fishery model (referred henceforth as the basic model) based on Béné et al. (2001), and an approximation of its viability kernel computed using VIKAASA are presented. In short, this problem concerns the "viability" of a fishery in which a single fish stock is being exploited by a fishing fleet. Viability here means the ability to maintain the fishery within the limits of ecological, economic and social objectives for an indefinite period. The basic model also provides the underlining platform for the viability models developed in Chapters 3 and 4, making this section a good introduction to viability modelling and analysis to be undertaken in the following chapters, without getting into too much detail.

\subsubsection{Basic model}

The elements of the model are outlined in Box 3 following the numbering used in Box 2, so that one can verify that each of the VIKAASA requirements is met.

\section{Box 3. Basic model specifications}

1. The model is concerned with an infinite time horizon, so $\Theta=[0, \infty)$.

2. The system is described by two state variables: fishable biomass (or simply "biomass") $x(t)$ and fishing effort $e(t)$. Effort is exerted by the 
fishing fleet to extract the resource (i.e. fish) at time $t$. This is a fixed fleet-size model, so there is no variation in capital to consider.

A "catchability coefficient" $q_{x}$ is defined to determine the quantity of biomass that each unit of effort extracts, relative to the total size of the biomass at the time. Thus, the harvest rate at time $t$ is

$$
h_{x}(t)=q_{x} e(t) x(t)
$$

3. Three viability constraints are given. The first constraint requires the regulator to maintain what is herein referred to as the ecological sustainability of the fishery, by ensuring that

$$
x(t) \geq x_{\min } \quad \forall t \in \Theta
$$

where $x_{\text {min }}>0$ is the safe minimum biomass level (SMBL). Below this level, the risk of resource extinction becomes unacceptably high and authorities will have to close the fishery.

The second constraint is concerned with what is henceforth referred to as the economic sustainability of the fishery, by guaranteeing that the fishing fleet's profits are non-negative at all times,

$$
\pi(t)=p_{x} h_{x}(t)-c e(t)-C \geq 0 \quad \forall t \in \Theta
$$

where $p_{x}$ is the price of a unit of biomass (fixed in this model), and as explained above, $h_{x}(t)$ is the harvest rate, making $p_{x} h_{x}(t)$ the fleet's revenue flow. $C$ is some fixed cost, and $c$ is a variable cost for each unit of effort. 
The third constraint concerns minimum and maximum levels of effort. The minimum level $e_{\min }$ is determined by social requirements for the preservation of employment and maintenance of traditional fishing communities ${ }^{16}$. The maximum level $e_{\max }$ is determined not by any normative considerations but rather by the physical capabilities of the fishing fleet. Given that there is no variation in capital and labour, it is supposed that maximum effort is constrained by a fixed production capacity. So, overall, the level of effort is bounded as follows:

$$
e(t) \in\left[e_{\min }, e_{\max }\right] \quad \forall t \in \Theta
$$

where the rate at which effort can change is also constrained

The constraint set $K$ in which biomass and effort must remain, reads as follows:

$$
K=\left\{\left[x_{\min }, x_{\max }\right] \times\left[e_{\min }, e_{\max }\right]\right\} \cap\left\{(x, e): p_{x} q_{x} e x-c e-C \geq 0\right\}
$$

It should be noted that there is no explicit upper limit on $x$. However, VIKAASA requires that both lower and upper limits be specified for all state variables. Here, $x_{\max }=L_{x}$ is specified because the fish stock's biomass cannot exceed the environment's carrying capacity.

4. It is supposed that so long as the ecological and economic sustainability of the system are maintained, regulatory instruments can be used to increase or decrease the level of effort exerted by the fleet. Thus, the system can be modelled as having a single scalar control, $u(t) \in \mathbb{R}$, which determines effort variation, i.e. $u(t)=\dot{e}(t)$. 
5. Effort variation is bounded by $U=\left[\delta^{-}, \delta^{+}\right]$, where $\delta^{-}<0$ and $\delta^{+}>0$. Thus, where $e(t)$ is too high (entailing imminent extinction), or too low (meaning that fishing will soon be unprofitable), it may not be possible to increase or decrease $e(t)$ fast enough (depending on the sizes of $\delta^{-}$ and $\delta^{+}$, which determine to speed of changes of $\left.e(t)\right)$ to maintain the viability of the system.

6. The fish population levels $x(t)$ are governed by a logistic differential equation,

$$
\dot{x}(t)=r_{x} x(t)\left(1-\frac{x(t)}{L_{x}}\right)-h_{x}(t) .
$$

The resource grows at a rate proportional to $r_{x}$, up to the limit carrying capacity $L_{x}$ of the resource's environment, less the harvest rate $h_{x}(t)$ defined by (2.8).

As mentioned in 4 and 5 , effort variation is given by the differential inclusion,

$$
\dot{e}(t)=u(t) \in U \equiv\left[\delta^{-}, \delta^{+}\right]
$$

This inclusion represents bounds on the speed at which the regulator can change fishing effort. This may reflect the regulator's policy for "smooth" fishing effort adjustments determined by $\delta$.

For VIKAASA to compute the viability kernel, all parameters including the boundaries of all dynamic variables must be assigned numerical values. The base parameter set considered for the computation of the basic model's

\footnotetext{
${ }^{16}$ It is noted that constraining $e(t)$ from below may be redundant given the constraint for profit $\pi(t)$.
} 
viability kernel is presented in Table 2.1. These parameters have been chosen so that the system is consistent with known characterisations of actual fisheries ${ }^{17}$, and to ensure that both viable and non-viable regions are to be found in the constraint space.

Table 2.1: The base parameter set for the basic model

\begin{tabular}{lll}
\hline \hline Description & Symbol & Value \\
\hline \hline Intrinsic growth rate & $r_{x}$ & 0.4 \\
Catchability coefficient & $q_{x}$ & 0.5 \\
Limit carrying capacity & $L_{x}$ & 600 \\
Price & $p_{x}$ & 4 \\
Variable cost & $c$ & 10 \\
Fixed cost & $C$ & 150 \\
SMBL & $x_{\min }$ & 60 \\
Minimum effort & $e_{\min }$ & 0.1 \\
Maximum effort & $e_{\max }$ & 1 \\
Maximum effort variation & $\delta$ & 0.01 \\
\hline
\end{tabular}

Thus, with these parameter values the dynamical system - (2.13) and (2.14) - becomes

$$
\left.\begin{array}{l}
\dot{x}(t) \in 0.4 x(t)\left(1-\frac{x(t)}{600}\right)-0.5 e(t) x(t) \\
\dot{e}(t) \in U=[-0.01,0.01]
\end{array}\right\},
$$

\footnotetext{
${ }^{17}$ For instance, the SMBL is set equal to the hard limit, i.e. $x_{\min }=\frac{L_{x}}{10}$, which is commonly implemented in fisheries worldwide.
} 
and the viability constraint set reads

$$
K=\{[60,600) \times[0.1,1]\} \cap\{(x, e): 2 e x-10 e-150 \geq 0\} .
$$

The state-space of interest is illustrated in Figure 2.1 by the "rectangle", which delimits the hyper-rectangle $\hat{K}^{18}$. Recall from item 3 in Box 2 that the bounds (i.e. lower and upper limits) on the dynamic variables are defined by $\hat{K}$. The downward-sloping curve gives the zero-profit line. Combinations of fish biomass and fishing effort below this line yield negative profits and are therefore not viable by definition, i.e. the economic constraint (2.10) is violated. The domain of the viability constraints $K \subset \hat{K}$ (or the viability space), is delimited by the economic constraint and the maximum fishing capacity (i.e. the horizontal line at $e=1$ ).

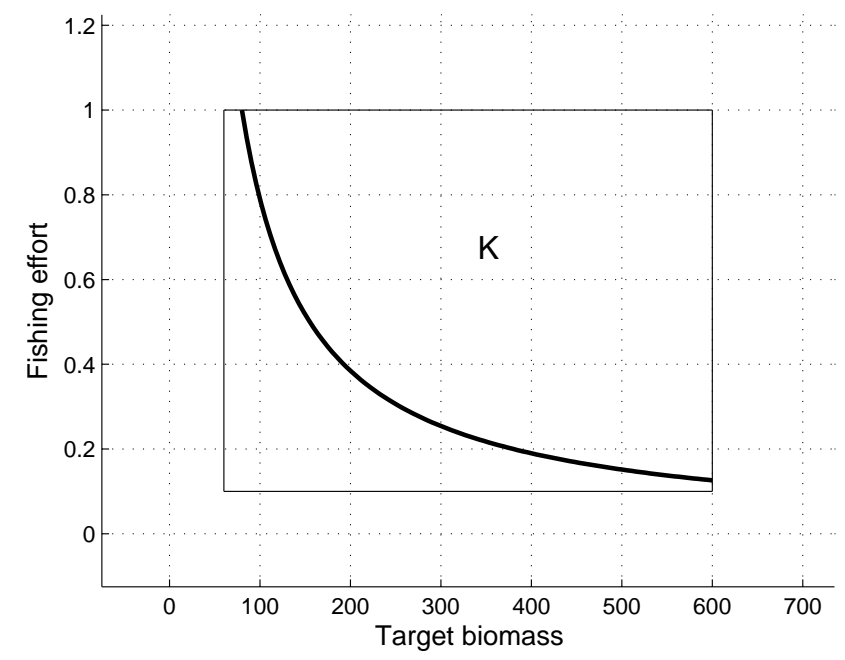

Figure 2.1: An illustration of the constraint set $K$ for the basic model.

\footnotetext{
${ }^{18}$ Chapter 3 deals with a viability kernel in a three-dimensional (3D) space, so $\hat{K}$ is delimited by a "box".
} 
Thus, it is of particular interest to determine the combinations of fish biomass and fishing effort in the constraint space that are associated with viable trajectories in $K$ and thus with viable control strategies. Note that this information is contained in the viability kernel. In what follows, the viability kernel approximated by VIKAASA for the basic model is presented.

\subsubsection{Presenting the "population viability" kernel}

As a starting point, the viability kernel computed without the economic constraint (2.10) or simply the population viability kernel is presented first ${ }^{19}$. The population viability kernel is represented by the shaded area (in yellow) in Figure 2.2a. By definition, combinations of target biomass and fishing effort in the shaded area are associated with viable paths, given the set of admissible controls $U$, that will always remain inside the rectangle ${ }^{20}$. Some of these viable paths are displayed in Figure 2.2b. Note that these paths (in yellow) remain inside the rectangle and converge to steady states indicated by the dashed line. This line represents the "sustainable equilibria" and corresponds to combinations of fish biomass and effort that guarantee an extraction rate that equates the reproduction rate of the resource stock $^{21}$.

Additionally, a ceiling on fishing effort is observed in Figure 2.2a, which is below its upper bound. Regardless of the state of fish biomass, levels of effort

\footnotetext{
${ }^{19}$ Here, only the sustainability of the fish stock or population is considered and hence the use of the term "population". Since the zero-profit line always lies above $e_{\min }$ (given the chosen parameter values), the social constraint is always guaranteed.

${ }^{20}$ Note that here the constraint set and the hyper-rectangle are identical, i.e. $K=\hat{K}$ due to the omission of the profitability condition (2.10).

${ }^{21}$ Equivalently, what is produced as surplus is harvested so the resource base remains constant. This is the basis for the use of the term "sustainable" to describe the equilibria.
} 


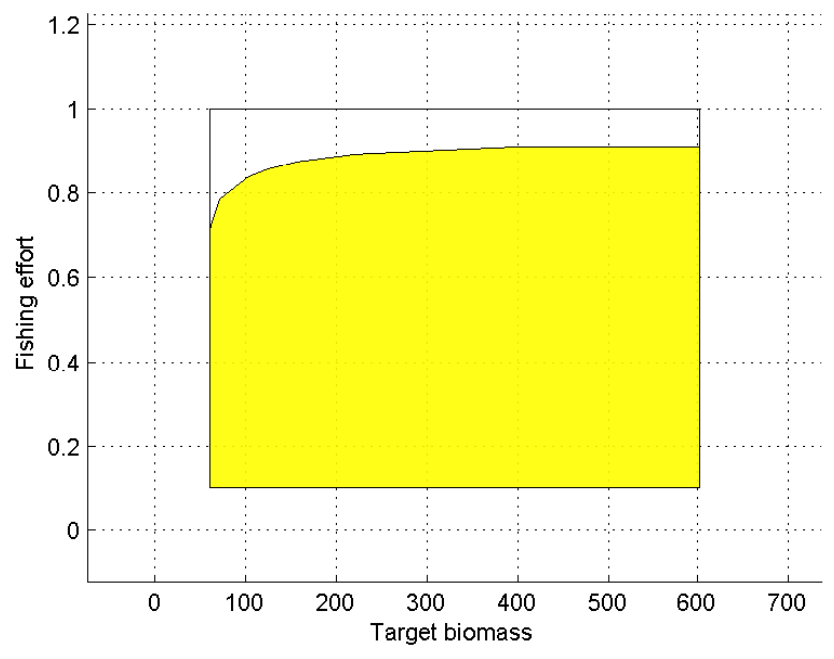

(a) Population viability kernel

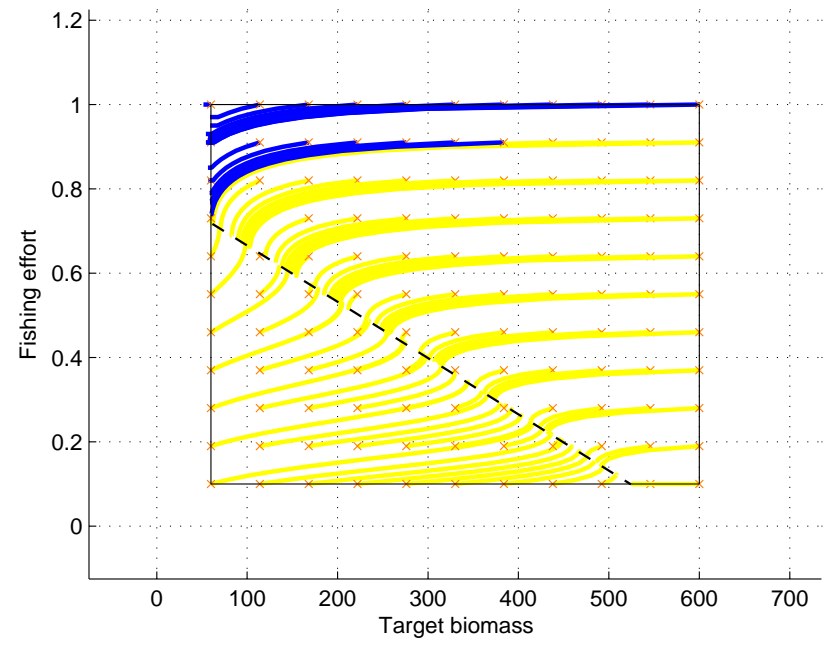

(b) Viable paths are yellow and non-viable paths are blue

Figure 2.2: Population viability kernel with viable and non-viable trajectories for the basic model. 
above this ceiling (i.e. in the unshaded area) are associated with initial states that are non-viable. More specifically, starting above the ceiling results in the resource stock being driven below its SMBL. This is confirmed in Figure $2.2 \mathrm{~b}$, with the relevant trajectories (in blue) shown to leave the rectangle via the fish biomass' lower bound.

\subsubsection{Presenting the "co-viability" kernel}

Now, the economic constraint is considered when computing the viability kernel for the basic model. To distinguish this kernel from the population viability kernel, the former is called the co-viability kernel because it considers all of the objectives conjointly. The co-viability kernel is presented in Figure 2.3a. Note that the co-viability kernel closely resembles the (partial) viability kernel presented in Fig. 3 in Béné et al. (2001). There is a noticeable change in the size and shape of the co-viability kernel relative to the population viability kernel. It is observed that a rounded area at the bottom of the diagram becomes non-viable as a consequence of imposing the economic constraint. More specifically, fishing is not profitable in this area because there is either not enough fish in the fishery or too little effort being exerted, or a combination of these two factors.

Additionally, the ceiling on fishing effort observed in Figure 2.3a is slightly lower than that shown in Figure 2.2a, meaning that some high fishing effort states in the population viability kernel are no longer viable now that the economic constraint is considered. This can be explained as follows. Although VIKAASA is able to find a control strategy that guarantees the ecological sustainability of the fishery, such strategy is insufficient to slow the system to a steady state before hitting the zero-profit line, see Figure 2.3b. Moreover, 


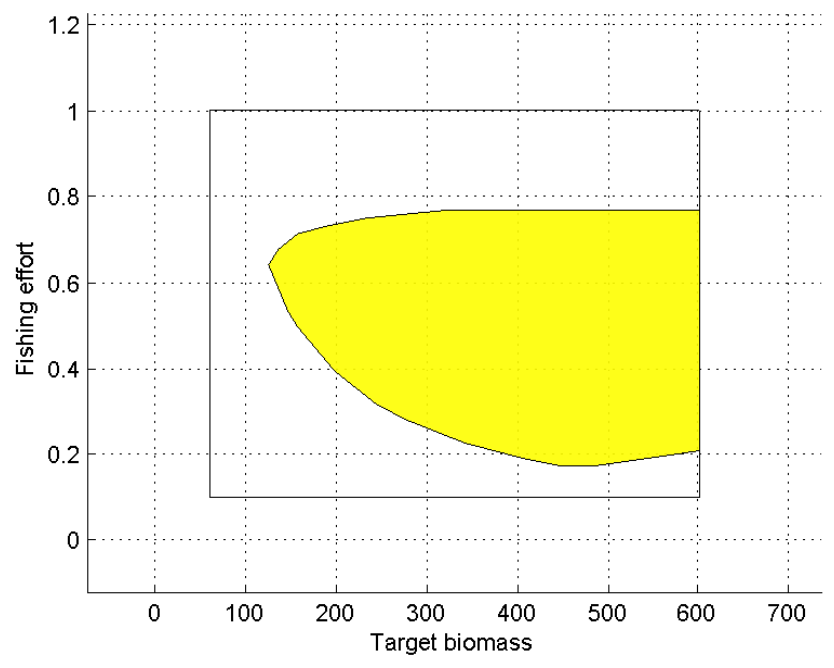

(a) Co-viability kernel

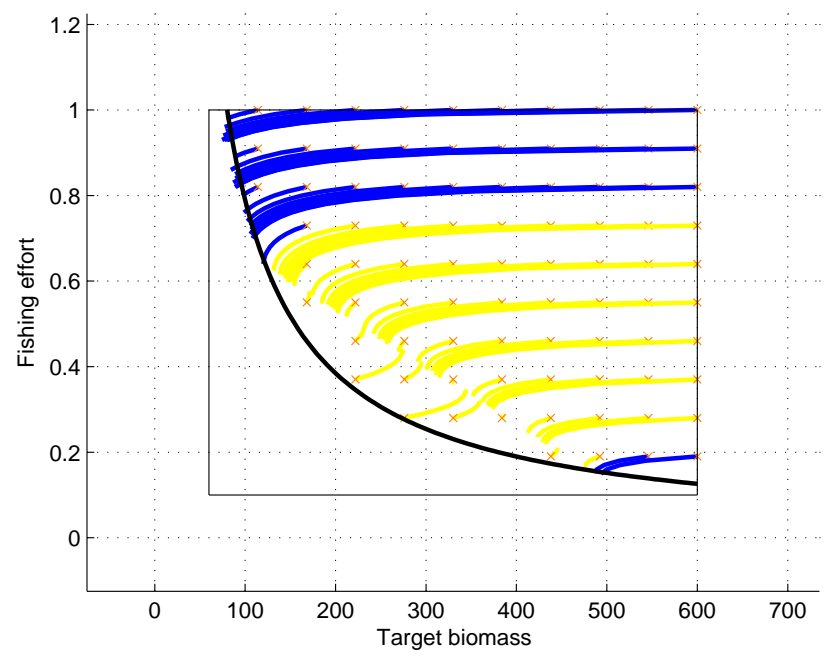

(b) Viable paths are yellow and non-viable paths are blue

Figure 2.3: Co-viability kernel with viable and non-viable trajectories for the basic model. For illustrative purpose, the zero-profit line is shown in panel (b). 
it is worth noting that starting with combinations of $x$ and $e$ on or above the zero-profit line does not guarantee non-negative profits for all times thereon. Thus, the state that the system will be steered towards depends on the system's dynamics $f(x, u)$ and its current position. So if the fishery is at a point where high fishing effort is exerted, the best strategy determined by VIKAASA might be to reduce $e$ by its maximum amount in order to conserve the fish stock. However, by doing so the rate of harvest might be too low to yield non-negative profit.

The existence of non-viability in the constraint set is an interesting case because there is a clear need to regulate the fishery and to anticipate the system's dynamics in order to avoid a crisis. Crisis here being either a nonprofitable or over-exploited fishery, or both. This case is known as partial viability and one that has received an analytical treatment in Béné et al. $(2001)^{22}$. Here, it is a result of assuming that the intrinsic growth rate is lower than the catchability coefficient (i.e. $r_{x}<q_{x}$ ) as well as the high rigidity imposed on the speed of fishing effort adjustment. The former implies that the fish stock's intrinsic growth rate is not sufficiently high to sustain high fishing effort. Furthermore, the latter limits the regulator's ability to reduce fishing effort by the necessary amount in order to stabilise the system within the realms of viability.

Note that if the converse of the first assumption is assumed (i.e. $r_{x}>q_{x}$ ) then viability holds everywhere in $K$, see Figure 2.4. The fishery can thus sustain itself and there is no need for intervention by the fishery manager. This case is called global viability in Béné et al. (2001) and reflects the mere

\footnotetext{
${ }^{22}$ Béné et al. (2001) propose the necessary and sufficient conditions for the existence of partial viability.
} 
fact that the maximum fishing capacity is too moderate to put the stock at risk of over-exploitation. Similarly, it can be easily shown that the viability space also expands when increasing the bounds on fishing effort variation. i.e. by specifying a larger value of $\delta$.

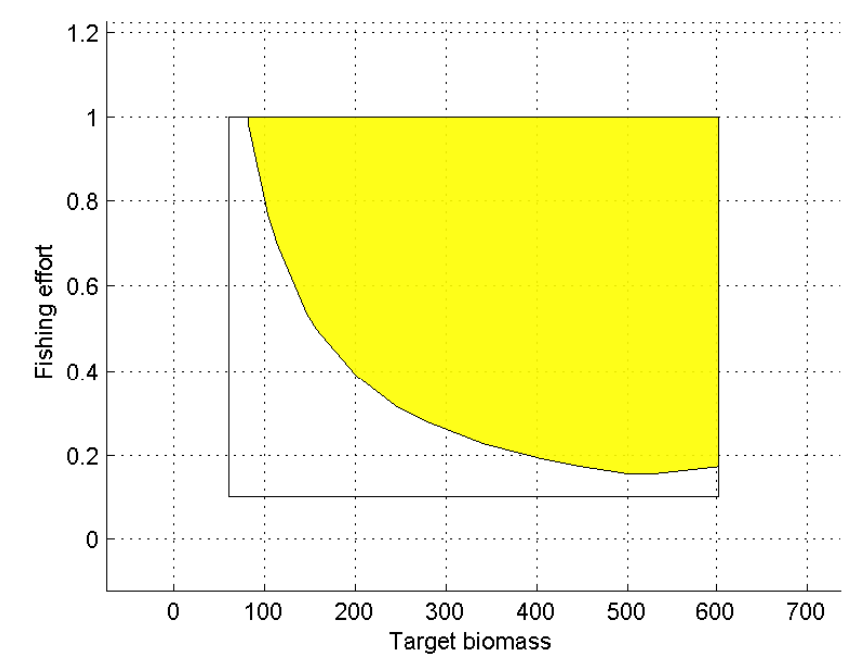

Figure 2.4: Co-viability kernel with $r_{x}>q_{x}$.

\subsubsection{Policy advice}

The preceding sub-section presented the co-viability kernel (or simply the "viability kernel"), which is the solution to the simple fishery's viability problem. Knowing the viability kernel, the fishery manager is aware of the locus of states in which the fishery can continue to exist, for a given "strength" of implementable controls. From the manager's point of view, the kernel holds invaluable information that align policy decisions with sustainable fishing outcomes. In effect, the viability kernel defines the boundaries of allowed fishing behaviour, within which the fleet can set level(s) of fishing effort to 
be exerted as it sees fit. The fishery manager's task is therefore to make sure that the fishery is maintained within these boundaries.

\subsection{Summary}

This chapter has provided a brief introduction to viability theory, which designs and develops mathematical and algorithmic methods for analysing dynamical systems facing constraints. Viability theory therefore provides an appropriate tool for solving problems of sustainability particularly here in the case of a fishery, which is of interest to this thesis.

This chapter has also introduced VIKAASA, a computer application capable of solving a certain class of viability problems by approximating the viability kernel, which delineates the locus of states from which the continued existence of a dynamical system is guaranteed. The application does have its share of limitations. Nonetheless, as demonstrated by its developers and again in this chapter, VIKAASA has delivered reliable results for an array of viability problems whose kernel properties are known a priori.

A stylised fishery model (i.e. basic model) has also been presented, which provides the basis for the viability models developed in the following chapters. As an introduction to viability analysis to be conducted later, the basic model's viability kernel approximated by VIKAASA is presented and briefly discussed. The viability kernel is essential for a regulator as it provides policy-relevant information for the sustainable management of the fishery.

The next chapter extends the basic model to include an additional fish stock, which is the by-catch (or by-product) of harvesting the target fish stock. 


\section{Chapter 3}

\section{A viability analysis of an input-controlled fishery}

\subsection{An overview}

The previous chapter introduced the mathematical concept of viability kernel to deal with the consistency between constraints and controlled dynamics. It also presented VIKAASA, an application developed to numerically approximate the kernel for a sub-set of viability problems. Using VIKAASA, the present chapter applies viability theory to a two-species fishery model with the aim of exhibiting the bio-economic configurations required to maintain the fishery in a perennial state.

A non-selective fishery (referred herein as the by-catch fishery) characterised by a by-catch production process is considered. This is a feature of most multi-species fisheries and one that is managed, in the current chapter, through direct controls on fishing input, i.e. fishing effort. The bio-economic model presented here extends the basic model described in the previous chap- 
ter with the noticeable inclusion of a by-catch fish stock. This model is referred henceforth as the input-controlled fishery model.

The problem here concerns the sustainable management of a by-catch fishery. One of the reasons for the by-catch problem introduced in Chapter 1 is the failure to consider the co-dynamics of jointly harvested fish stocks in policy decisions. This may result in certain slower growing stocks being over-fished when exploited at rates appropriate for the target stocks. Thus, viability here refers to the materialisation of sustainability objectives, such as those defined in the basic model (Section 2.4.1) coupled with an ecological constraint for the by-catch stock.

In what follows, the theoretical framework of the input-controlled fishery model is presented in Section 3.2, which defines the system dynamics and the viability constraints. For convenience, the mathematical symbols and their descriptions for the model developed here are presented in the List of Symbols, which is available towards the end of this thesis. The model is calibrated in Section 3.3. Justification for the parameter values chosen is also provided. An analysis of the viability kernel approximated by VIKAASA for the input-controlled fishery model is documented in Section 3.4. Some policy advice is also provided before closing this chapter with some concluding remarks in Section 3.5. 


\section{$3.2 \quad$ A viability model}

\subsubsection{The system dynamics}

The system is described by three state variables: target biomass $x(t)$, bycatch biomass $y(t)$ and fishing effort $e(t)$, and is modelled over an infinite time horizon,

$$
t \in \Theta \equiv[0, \infty)
$$

\section{The population dynamics}

The dynamics of the biological variables are based on the surplus production approach or (dynamic) biomass approach often connected to Schaefer (1954), which has been widely adopted in economic fishery studies. The approach uses the Pearl-Verhulst logistic equation and describes the state of the population using only a single variable, i.e. biomass (Tahvonen, 2010). Notably, the surplus production approach has been criticised typically for its over-simplification assumptions and minor empirical relevance (Wilen, 2000). According to Townsend (1986), it is only suitable for "pedagogical" purposes. Nonetheless, the surplus production model is conveniently simple and removes the complexities associated with working with highly dimensional and parameterised age-structured models ${ }^{1}$. Furthermore, at this stage, modelling the population dynamics based on an aged-structured specification, such as in Beverton \& Holt (1957), is not sufficiently useful to compensate for the non-practicality, i.e. significant computational time and

\footnotetext{
${ }^{1}$ The age-structured approach divides the fish population into age classes or cohorts, and its development into primary factors such as reproduction, individual growth, natural mortality, and fishing mortality (Tahvonen, 2010).
} 
extreme multi-dimensionality of the kernel, of modelling multiple cohorts for both stocks in VIKAASA.

Suppose that the fishery of interest consists of two ecologically independent populations, with fishable biomasses $x(t)$ and $y(t)$, that are subject to logistic growth in continuous time,

$$
\left\{\begin{array}{l}
\dot{x}(t)=r_{x} x(t)\left(1-\frac{x(t)}{L_{x}}\right) \\
\dot{y}(t)=r_{y} y(t)\left(1-\frac{y(t)}{L_{y}}\right)
\end{array}\right.
$$

where $r_{x}, r_{y}, L_{x}$, and $L_{y}$ are all positive constants. By convention, $r_{x}$ and $r_{y}$ represent the intrinsic growth rates $^{2}$, and $L_{x}$ and $L_{y}$ are the environment's limit carrying capacities of stocks $x$ and $y$, respectively.

Now, suppose that the two population dynamics described by (3.2) are subject to withdrawals. Let the harvest rates (i.e. fishery aggregates) of of stocks $x$ and $y$ from the fishery be given by $H_{x}(t)$ and $H_{y}(t)$ respectively. The population dynamics now become,

$$
\left\{\begin{array}{l}
\dot{x}(t)=r_{x} x(t)\left(1-\frac{x(t)}{L_{x}}\right)-H_{x}(t) \\
\dot{y}(t)=r_{y} y(t)\left(1-\frac{y(t)}{L_{y}}\right)-H_{y}(t) .
\end{array}\right.
$$

Suppose that stock $x$ is the fish stock that is being targeted. This may be because stock $x$ is more valuable and/or abundant than stock $y$. The harvest rate of stock $x$ at time $t$ is assumed to be proportional to both its biomass and the level of fishing effort,

$$
H_{x}(t)=q_{x} e(t) x(t)
$$

\footnotetext{
${ }^{2}$ The intrinsic growth rate represents the maximum growth rate of the population, achieved at the smallest abundance levels. It is basically a balance between fecundity, mortality, maturation and growth (FLR Project, 2013).
} 
where $q_{x}$ denotes the catchability coefficient. The catchability coefficient links the level of fishing effort to the proportion of the stock removed (Pascoe, 1997). The product of the catchability coefficient and the level of effort is generally termed the fishing mortality,

$$
f_{x}(t)=q_{x} e(t)
$$

and it is expressed as a proportion of biomass.

Moreover, the fishery is characterised by a by-catch process. With each unit of fishing effort, the fishing fleet catches both fish stocks, $x$ and $y$, simultaneously. This may result from the combination of non-selective harvest technologies (e.g. bottom trawl) and over-lapping populations of marine species (Herrera, 2005). This makes harvesting like a joint production function where one species (i.e. stock $y$ ) is a byproduct of the production process of another (i.e. stock $x$ ).

Here, a linear by-catch production function is proposed,

$$
H_{y}(t)=\alpha H_{x}(t)
$$

where $H_{x}(t)$ is given by (3.4) and the parameter $\alpha>0$ denotes the by-catchtarget harvest ratio, which is a measure of the intensity of jointness of the production relationship.

It is worth noting that most fisheries are not characterised by pure joint production, but instead may be "mostly" joint. That is, the composition of the output mix may have some discretionary element. Agents may be able to increase the proportion of one species or another in the catch through varying their targeting behaviour. In such a situation, by-catch is less an ecological and technological problem, but also a function of fisher targeting behaviour 
(Abbott \& Wilen, 2009). Thus, in reality the by-catch-target harvest ratio may vary over time. For the sake of simplicity, here $\alpha$ remains fixed.

\section{Fishing effort adjustments}

Following Béné et al. (2001), it is proposed that the time variation of effort is described by the following differential inclusion,

$$
\dot{e}(t)=u(t) \in U \equiv[-\delta, \delta]
$$

where $\delta \geq 0$ is a constant that defines the maximum speed of fishing effort adjustment at each time realisation. The inclusion represents bounds on the speed of adjustment of effort. This may reflect the regulator's policy for "smooth" fishing effort adjustments. That is, it implies the continuity of effort with respect to time and thus rules out jumps in harvesting levels. Additionally, $-\delta<0<\delta$ is assumed, which means that effort can be kept constant (i.e. $\dot{e}=0$ ).

Thus, the system dynamics for the input-controlled fishery model is defined through the following system of differential inclusions,

$$
\begin{aligned}
& \dot{x}(t) \in r_{x} x(t)\left(1-\frac{x(t)}{L_{x}}\right)-q_{x} e(t) x(t) \\
& \dot{y}(t) \in r_{y} y(t)\left(1-\frac{y(t)}{L_{y}}\right)-\alpha q_{x} e(t) x(t) \\
& \dot{e}(t) \in U .
\end{aligned}
$$

Note that the system of differential equations (3.3), which describes the population dynamics for both stocks are now expressed in terms of differential inclusions. This is a consequence of assuming that the evolution of fishing effort is set-valued. So for a given $x(t)$, there is an array of possible controls 
to choose from in $U$ and hence have a set of points in the state-space that can be reached in the next instant. Given the by-catch production relationship (3.6), the evolution of the by-catch stock is consequently set-valued as well.

In what follows, the viability constraints are defined. These constraints delineate the boundaries within which dynamical system must remain in order to guarantee the continued sustainability of the fishery.

\subsubsection{The viability constraints}

Following Martinet et al. (2007), it is proposed that the sustainability of the fishery is represented by a set of ecological, economic and social constraints that the fishery must satisfy at all times. Each of these three constraints are described below.

\section{The ecological constraint}

This constraint concerns the ecological sustainability of the two resource stocks and requires that both $x$ and $y$ do not fall below their respective SMBLs, $x_{\min }>0$ and $y_{\min }>0$,

$$
\left.\begin{array}{l}
x(t) \geq x_{\text {min }} \\
y(t) \geq y_{\text {min }}
\end{array}\right\} \quad \forall t \in \Theta,
$$

where $\Theta$ is defined in (3.1). The interpretation of the SMBL used here is that below this level the risk of resource extinction becomes unacceptably high and authorities will move to close the fishery. 


\section{The economic constraint}

This constraint guarantees the economic sustainability of the fishing fleet and requires that the fishing activity remains profitable. Aggregate profit for the fishery is given by,

$$
\pi(t)=p_{x} H_{x}(t)+p_{y} H_{y}(t)-c e(t)-C,
$$

where $p_{x}$ and $p_{y}$ are constants and represent the unit prices of stocks $x$ and $y$, respectively ${ }^{3}$. Fixed cost which may include yearly lease of a vessel from its owner, insurance, taxes, depreciation and the appropriate opportunity costs is denoted by the constant $C$. The marginal cost of effort is fixed at $c$.

The economic constraint imposes a non-negative condition on profit,

$$
\pi(t) \geq 0 \quad \forall t \in \Theta
$$

As noted in Béné et al. (2001), this condition is more "conservative" than the conventional "shutdown" condition taught in the economic theory of the firm. The shutdown condition states that if revenue exceeds variable cost, the firm should continue to operate, at least in the short-run, because it is earning something towards its overheads, otherwise it should close down to avoid paying additional cost to its fixed cost, which it already loses by not producing (Begg et al., 2003).

Possible interpretations for condition (3.11) are presented in Krawczyk et al. (2013). One assumption is that fishermen do not amass any savings from their operations, and therefore cannot afford to run at a loss even for a

\footnotetext{
${ }^{3}$ In some situations, $p_{y}$ could be negative to represent a penalty incurred by the fisherman for catching the by-catch species. This could though be a politically problematic solution because of underreporting or non-reporting and dumping possibilities of stock $y$.
} 
brief period. Another possible interpretation is that it is a political requirement - i.e. that the fleet's cooperation with the regulator on fishing levels is contingent on continued profitability, so that if fishing became unprofitable, the regulator would no longer have any influence.

\section{The social-physical constraint}

The social constraint concerns the preservation of employment and maintenance of traditional fishing communities. This requires that the regulator maintains fishing effort above a minimum threshold, $e_{\min }>0$. This guarantees minimum employment and activity in the fishery. As noted in the previous chapter, the values of the economic parameters chosen may result in the zero-profit line to lie above $e_{\min }$ making this social condition redundant. Nonetheless, for guaranteed activity in the fishery, fishing effort must take on a positive value.

Moreover, the bounds imposed on the speed at which fishing effort can be adjusted, i.e. condition (3.7), may reflect some social (and political) cost, which limits the number of vessels, and hence employment, from leaving the fishery during any period (Martinet et al., 2007) ${ }^{4}$.

Additionally, given that there is no variation in capital and labour, it is supposed that effort is constrained by a fixed production capacity, i.e. there is a maximum level of effort, $e_{\max }$ that can be exerted. Fishing effort is therefore bounded by,

$$
e(t) \in\left[e_{\min }, e_{\max }\right] \quad \forall t \in \Theta
$$

\footnotetext{
${ }^{4}$ This interpretation differs from the "capital inertia" explanation often encountered in the fisheries economics literature (Martinet et al., 2007).
} 
Thus, using (3.7), (3.9), (3.11) and (3.12), the viability constraint set is defined by,

$$
K \equiv\left\{\begin{array}{l|c}
(x, y, e, u): & \begin{array}{c}
x(t) \geq x_{\min } \\
y(t) \geq y_{\min } \\
\pi(t) \geq 0 \\
e(t) \in\left[e_{\min }, e_{\max }\right] \\
u(t) \in U
\end{array}
\end{array}\right\}
$$

\subsubsection{The viability kernel}

A commonly raised question in any viability analysis is whether the system dynamics (3.8) are compatible with the set of viability constraints (3.13). Here, compatibility means that with the predetermined system's dynamics, the system's evolutions will always satisfy the constraint set $K$. By answering this question, one can delineate the set of initial states from which there exist viable evolutions that respect the entire set of constraints. This is known from the previous chapter as the viability kernel or the solution to the viability problem, and it is defined by ${ }^{5}$,

$$
\mathcal{V} \equiv\left\{(x(0), y(0), e(0)): \mid \begin{array}{c}
\exists u(\cdot) \text { and }(x(\cdot), y(\cdot), e(\cdot)), \\
\text { starting from }(x(0), y(0), e(0)) \\
\text { satisfying dynamics }(3.8) \\
\text { and constraints }(3.13) \\
\forall t \in \Theta
\end{array}\right\} .
$$

\footnotetext{
${ }^{5}$ To unburden the notation, $\mathcal{V}$ is used instead of $\mathcal{V}_{F}(K)$ to denote henceforth the viability kernel.
} 


\subsection{The model calibration}

In order to compute the viability kernel using VIKAASA, which does so numerically, all parameters must be assigned numerical values. This also applies to the boundaries of all dynamic variables. In what follows, the assigned values for all parameters and boundaries are presented, as well as some discussion on the rationale behind these values.

\subsubsection{The base parameter set}

It is assumed that target stock $x$ is more productive (i.e. $r_{x}>r_{y}$ ) and has a larger carrying capacity relative to the by-catch stock $y$ (i.e. $L_{x}>$ $\left.L_{y}\right)$. The carrying capacity for stock $x$ is set at 600 , which is twice that of $y$. If the unit of measure of biomass is denominated in tonnes, then it is acknowledged that the order of magnitude of these values are far from what is commonly associated with biomass. However, for the purpose of the analysis in Section 3.4, the relative value of the carrying capacities is far more important than their respective values in absolute terms. The latter has no impact on the results other than changing the scale of measurement. This does not apply, however, to the intrinsic growth rates, which are set at 0.4 and 0.2 for stocks $x$ and $y$, respectively. These are not unreasonable values given that, for instance, Polacheck et al. (1993) and Chen \& Andrew (1998) estimate the intrinsic growth rate for the northern Namibian Cape hake (Merluccius capensis and M. paradoxus) to be about 0.37.

Using these biological parameters, Figure 3.1a illustrates the time course, without withdrawals, for fish stocks $x$ (in blue) and $y$ (in red). The population size, or in this case the biomass (weight equivalent) for both stocks, 
starting at some small initial states, grow at an approximately exponential rate. As the environment's carrying capacity is approached (e.g. competition for limited food supply or space), their growth rates slow until an equilibrium limit is reached, where growth stops. The solution curves have the characteristic ogive shape of the logistic growth curve (Clark, 1990). The solution curve of the logistic growth equation(s) (3.2) is defined formally by (A.2).

The population dynamics of $x$, with withdrawal, is simulated for different values of $q_{x}$ and $e$, which are held fixed over time. The results are presented in Figure 3.1b. The simulated dynamics are most interesting when $q_{x}=0.5$ because for high values of effort, the target stock eventually depletes over time. This is shown in the bottom two panels. It is worth noting that the corresponding fishing mortality rates, i.e. $0.5 \times 0.8=0.4$ and $0.5 \times 1=0.5$ from (3.5), are at least greater than $r_{x}=0.4$ used in the simulation. This confirms that for the logistic model, the stock is driven (asymptotically) to zero if fishing mortality is maintained at a level (at least) greater than the intrinsic growth rate (Clark, 1990) ${ }^{6}$. For this reason, the value chosen for the catchability coefficient of stock $x$ is $q_{x}=0.5$.

It is worth noting here that the biological parameters $\left(r_{x}, r_{y}, L_{x}\right.$ and $\left.L_{y}\right)$ and the technical parameter $\left(q_{x}\right)$ can be estimated by fitting the surplus production model (3.3) to a time series of catches and abundance indices, which are available for most commercially exploited fish stocks. Although parameter estimation is not performed in this study, Appendix B provides some discussion as to how this can be carried out.

Similarly, the population dynamics of stock $y$ is simulated for different

\footnotetext{
${ }^{6}$ Equivalently, $e$ cannot exceed the ratio $\frac{r}{q}$. This ratio is called the "bio-technical productivity" of the population in Clark (1990)
} 


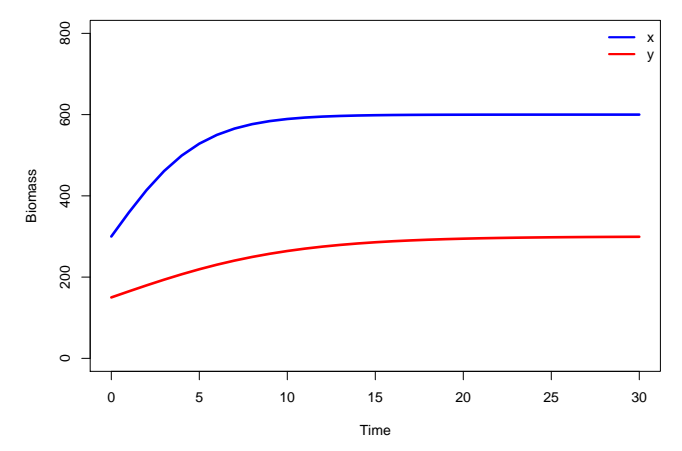

(a) Typical solution curves for stocks $x$ and $y: r_{x}=0.4, r_{y}=0.2, L_{x}=600$, $L_{y}=300, x(0)=300$ and $y(0)=150$.
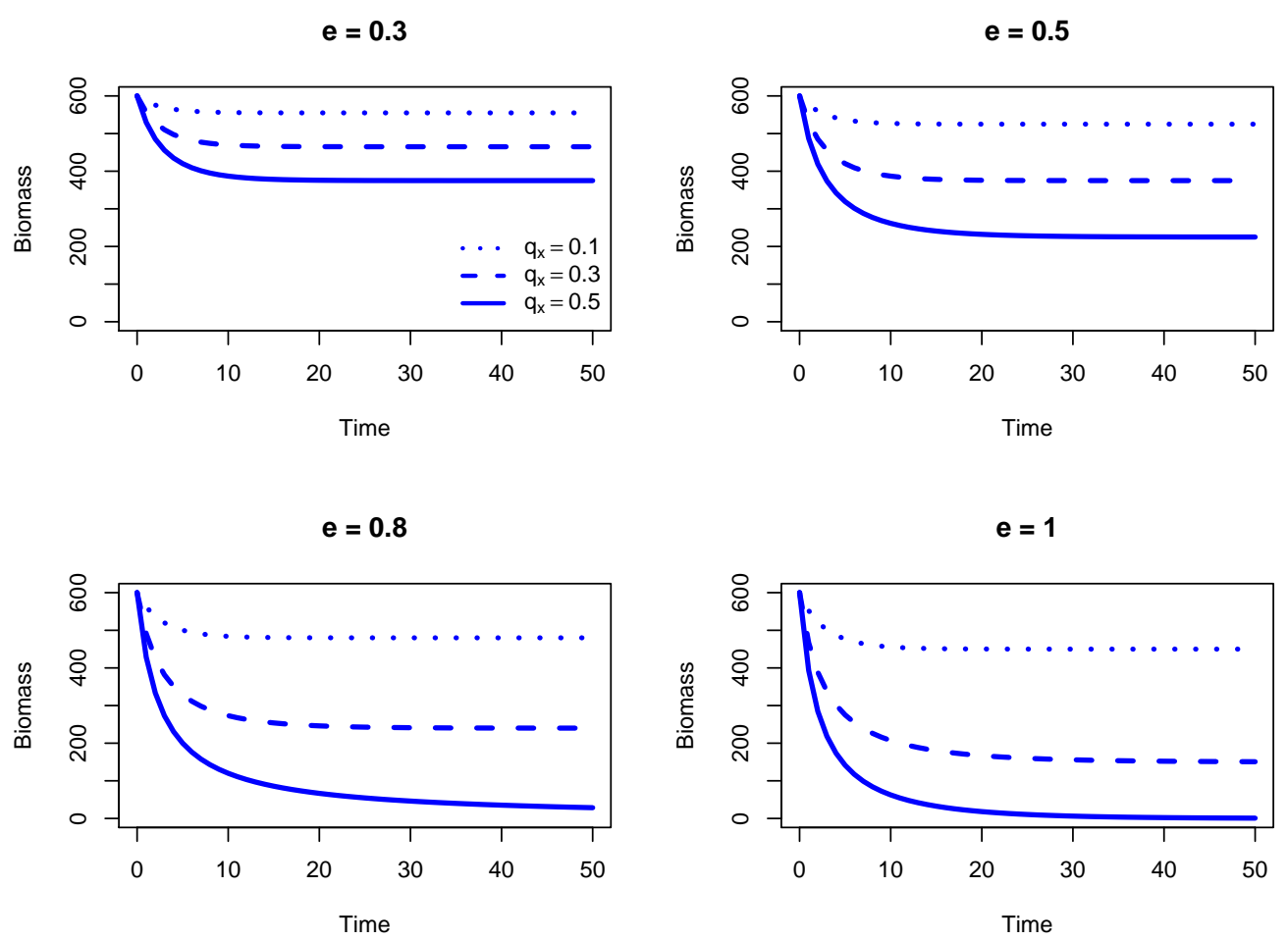

(b) Population dynamics of stock $x$ with constant effort: $r_{x}=0.4, L_{x}=600$ and $x(0)=600$.

Figure 3.1: Population dynamics simulated for selected parameter values. 
levels of effort and the by-catch-target harvest ratio $\alpha$, and presented (in red) in Figure 3.2. For $\alpha=0.1$, stock $y$ is never at risk of depletion. However, if $\alpha=0.3$, stock $y$ is depleted over time for low to moderate levels of effort. To capture these two behaviours, a value of $\alpha$ between 0.1 and 0.3 is chosen, i.e. $\alpha=0.2$. This seems to be in the right neighbourhood considering that, for example, $27 \%$ of catch of trevally (Pseudocaranx dentex) is reported as by-catch when tarakihi (Nemadactylus macropterus) is targeted using bottom-trawl in New Zealand's fisheries management area 2 (Bentley, 2010). An interesting observation is that the by-catch biomass exceeds the target biomass (in blue) at high levels of $e$. This is noted in the bottom two panels. This may be explained by the mere fact that at high effort levels, there is sufficient reduction in the target biomass resulting in lower target yield $H_{x}$. Combination of low $\alpha$ and sustained low $H_{x}$ keeps by-catch harvest substantially low over time, allowing for the by-catch biomass to exceed the target stock's biomass at some point in time.

As for the economic parameters, the ex-vessel unit price of stock $x$ is set at $\$ 4$ and for stock $y$ it is set much lower at $\$ 2$. Stock $x$ is the more valuable fish stock and therefore it is the stock that is targeted. The unit cost of effort $c$ is equal to $\$ 10$ and the fixed cost is set at $\$ 150$. These values ensure that there are areas associated with both negative and positive profits in the state-space.

The calibrated parameter values, which are summarised in Table 3.1, in broad terms characterise a typical multi-species fishery in which the target stock $x$ is more valuable and it is associated with higher catch rates (i.e. because it is more abundant) than the by-catch stock. The latter is considerably less productive than the former. So the by-catch stock is at risk of 

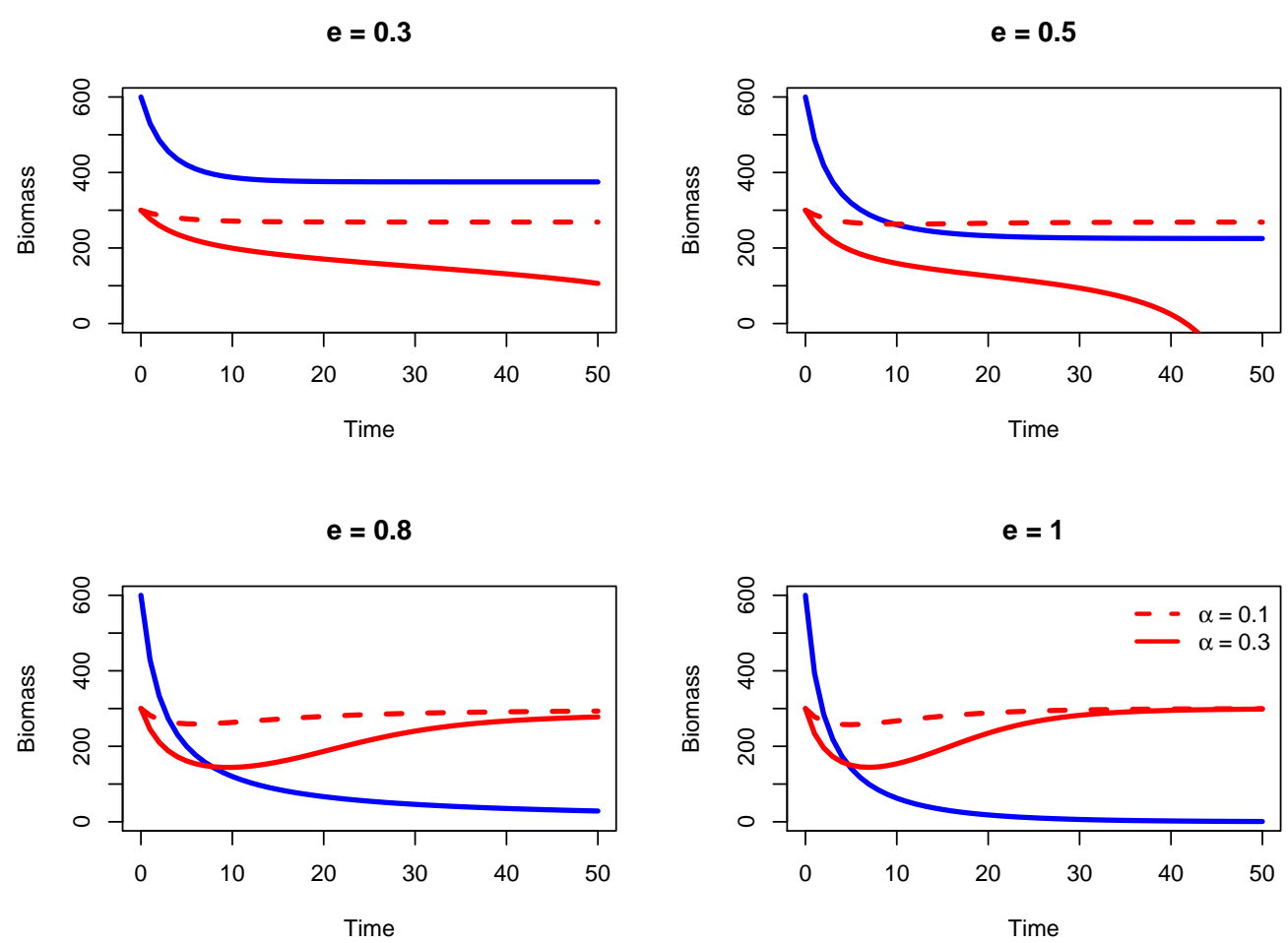

Figure 3.2: Population dynamics of the by-catch stock $y$ (in red) for different values of $\alpha$ and $e$. The population dynamics of stock $x$ is in blue: $r_{x}=0.4$, $q_{x}=0.5, L_{x}=600, L_{y}=300, x(0)=600$ and $y(0)=300$.

extinction when exploited at rates appropriate for the target stock. Thus, it is of particular interest to use viability theory to determine the loci of states from which the regulator can make the necessary adjustments that guarantee the good health of both fish stocks, while meeting its other economic and social responsibilities. 


\subsubsection{The boundaries}

As mentioned earlier, VIKAASA requires that both the lower and upper limits be specified for all state variables (refer to item 3 in Box 2).

For the biological variables $x$ and $y$, their lower limits are set by the ecological constraint (3.9). That is, the target and by-catch biomasses cannot fall below their respective SMBLs, $x_{\min }$ and $y_{\min }$. Here, the SMBLs are set at $10 \%$ of the stock's carrying capacities,

$$
\left.\begin{array}{l}
x_{\min } \equiv 0.1 L_{x} \\
y_{\text {min }} \equiv 0.1 L_{y}
\end{array}\right\} .
$$

New Zealand's Harvest Strategy Standard for instance, sets a default hard limit below which the stock is deemed to be collapsed, and the fishery is considered for closure (Ministry of Fisheries, 2008). This is consistent with the definition of the SMBL stated earlier in Section 3.2.2, and therefore the reason for setting SMBL equal to the hard limit for both stocks. The default hard limit is equal to $10 \%$ of $B_{0}$ (Ministry of Fisheries, 2008) ${ }^{7}$.

As for the two stocks upper limits $x_{\max }$ and $y_{\max }$, they are set equal to the fishery's carrying capacity for both fish stocks. The reason being, the size of either stock's biomass can never exceed their respective carrying capacity, even without fishing taking place as illustrated in Figure 3.1a.

The maximum level of fishing effort, $e_{\max }$ is set equal to unity, which reflects full (i.e. 100\%) capacity. It also ensures that fishing mortality (3.5) takes on a value that is never greater than 1 given the value chosen for $q_{x}$.

\footnotetext{
${ }^{7}$ In the fisheries literature, $B_{0}$ is widely used to denote the virgin or unexploited biomass, which is equivalent to the carrying capacity denoted by $L$ (with subscript $x$ or $y$ depending on which stock is being referred to) used in the logistic growth model.
} 
Note that the converse implies that $H_{x}>x$, which is a violation of the scarcity constraint, i.e. we harvest more than what is available. As for the minimum level of fishing effort $e_{\min }$, it is set rather arbitrarily equal to 0.1 or $10 \%$ of full capacity. Similarly, the limit on the speed of effort adjustment, $\delta$ is also set arbitrarily equal 1 percentage point.

Thus, the proposed boundaries for the input-controlled fishery model are presented below:

- target biomass should be between $10 \%$ and $100 \%$ of the stock's carrying capacity, i.e. $x(t) \in[60,600]$;

- bycatch biomass should be between $10 \%$ and $100 \%$ of the stock's carrying capacity, i.e. $y(t) \in[30,300]$;

- fishing effort should be between $10 \%$ and $100 \%$ of the fleet's maximum capacity, i.e. $e(t) \in[0.1,1]$; and

- fishing effort adjustment speed, i.e. the amount by which the regulator can change fishing effort between fishing periods $t$ will be between -1 and 1 percentage points, so $u \in[-0.01,0.01]$. 
Table 3.1: The base parameter set for the input-controlled fishery model.

\begin{tabular}{lllll}
\hline \hline Stock-specific & Target stock $(x)$ & \multicolumn{3}{l}{ By-catch stock $(y)$} \\
\hline \hline Description & Symbol & Value & Symbol & Value \\
\hline Intrinsic growth rate & $r_{x}$ & 0.4 & $r_{y}$ & 0.2 \\
Limit carrying capacity & $L_{x}$ & 600 & $L_{y}$ & 300 \\
Price & $p_{x}$ & 4 & $p_{y}$ & 2 \\
Catchability coefficient & $q_{x}$ & 0.5 & - & - \\
SMBL & $x_{\min }$ & 60 & $y_{\min }$ & 30 \\
\hline \hline Fishery-specific & & & & \\
\hline \hline Description & Symbol & Value & \\
\hline By-catch-target harvest ratio & $\alpha$ & 0.2 & \\
Variable cost & $c$ & 10 & \\
Fixed cost & $C$ & 150 & \\
Minimum effort & $e_{\min }$ & 0.1 & \\
Maximum effort & $e_{\max }$ & 1 & \\
Maximum effort variation & $\delta$ & 0.01 & \\
\hline
\end{tabular}


Specifications of the input-controlled fishery model developed in this chapter are summarised in Box 4, following the VIKAASA requirements outlined in Box 2.

\section{Box 4. Input-controlled fishery model specifications}

1. The model is concerned with an infinite time horizon, so $\Theta \equiv[0, \infty)$;

2. The system is described by three state variables: target biomass $x(t)$, bycatch biomass $y(t)$ and fishing effort $e(t)$;

3. The viability constraint set:

$K=\{[60,600] \times[30,300] \times[0.1,1]\} \cap\{(x, e): 2.2 e x-10 e-150 \geq 0\}$.

4. The control variable is represented by the scalar $u$;

5. The admissible control set is represented by $U=[-0.01,0.01]$;

6. The system's dynamics are represented by the following system of differential inclusions:

$$
\begin{aligned}
& \dot{x}(t) \in 0.4 x(t)\left(1-\frac{x(t)}{600}\right)-0.5 e(t) x(t) \\
& \dot{y}(t) \in 0.2 y(t)\left(1-\frac{y(t)}{300}\right)-0.1 e(t) x(t) \\
& \dot{e}(t) \in U .
\end{aligned}
$$




\subsection{A viability analysis}

In Chapter 2, the viability kernel for the single-species fishery problem was presented. Here, the analysis is extended to consider the case where the regulator is also concerned about maintaining the ecological sustainability of a by-catch fish stock, which is assumed to be jointly harvested with the target stock. It is of particular interest to learn what happens to the singlespecies kernel (Figure 2.3a) when accounting for the by-catch dynamics. This is considered in Section 3.4.2. Firstly, the viability kernel for the inputcontrolled fishery model described above is presented.

\subsubsection{Presenting the viability kernels}

The viability kernel is presented in Figure 3.3a. The box delimits the threedimensional projection of the viability constraint set $K \subset \mathbb{R}^{3}$ less the economic constraint (3.11), which is not displayed" . The shaded 3D body ("boulder") represents the viability kernel and contains the loci of initial states $[x(0), y(0), e(0)]$ from which there exists a control strategy $u \in U$ that keeps the system in $K$ forever. Those states outside the boulder cannot be controlled to remain in $K$ by the admissible set of controls $U$, so starting at any of these points eventually results in the system leaving $K$, in which case the fishery is considered to be in a "crisis" situation as defined earlier".

\footnotetext{
${ }^{8}$ Actually, the box is a three-dimensional projection of $\hat{K} \subset \mathbb{R}^{3}$, where $\hat{K}$ delimits the bounds on all three state variables $(x, y$ and $e)$.

${ }^{9}$ There are a number of papers (see Doyen \& Saint-Pierre (1997); Béné et al. (2001); Martinet et al. $(2007,2010))$ that have extended the viability approach to examine the viable restoration of bio-economic systems facing crisis situations. These papers use the concept of minimum time of crisis (Doyen \& Saint-Pierre, 1997) to analyse recovery prob-
} 


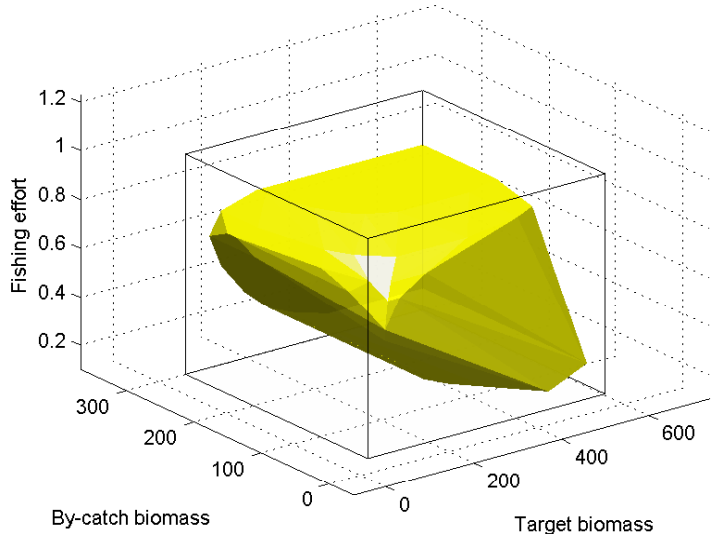

(a) Viability kernel "with" the economic constraint

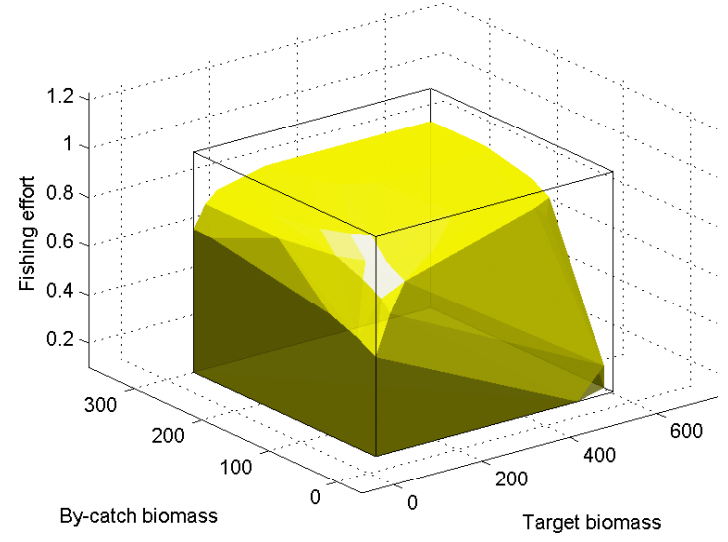

(b) Viability kernel "without" the economic constraint

Figure 3.3: A comparison of the viability kernel with and without the economic constraint.

The viability kernel generated without the economic constraint (3.11) is presented in Figure 3.3b. By comparing the two panels in Figure 3.3, the regulator obtains a general idea of which states violate the economic sustainability of the fishery. That is, starting at such states will result in negative cash flows for the fishing fleet taking effect at some point in time.

The by-catch management problem can also be analysed using $2 \mathrm{D}$ slices of the 3D kernel. Figure 3.4 shows such of the kernel presented in Figure 3.3a for each of the three dimensions. This provides a closer and more detailed lems. It is worth mentioning here that though the current analysis does not specifically consider recovery strategies, it is possible in VIKAASA to direct any initial state, starting from outside the viability kernel, to re-enter the kernel in finite time. This requires that the bounds, imposed on the speed of adjustment of the system's control(s), be relaxed. This implies that at least one of the viability constraints will be violated in finite time. 


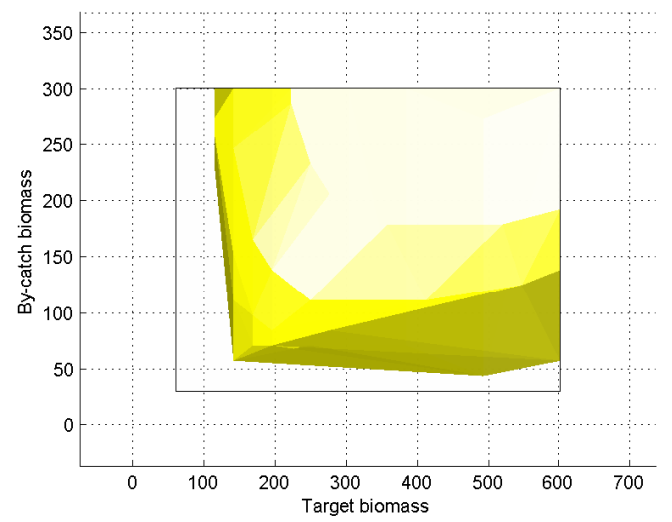

(a) Slice through all values of "fishing effort"

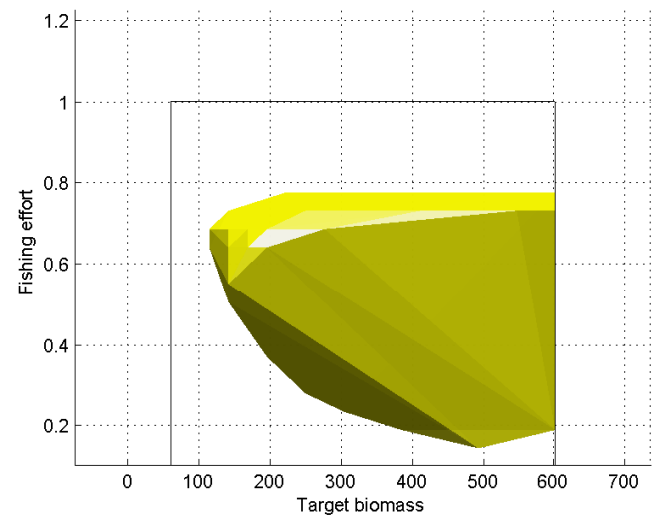

(b) Slice through all values of "by-catch biomass"

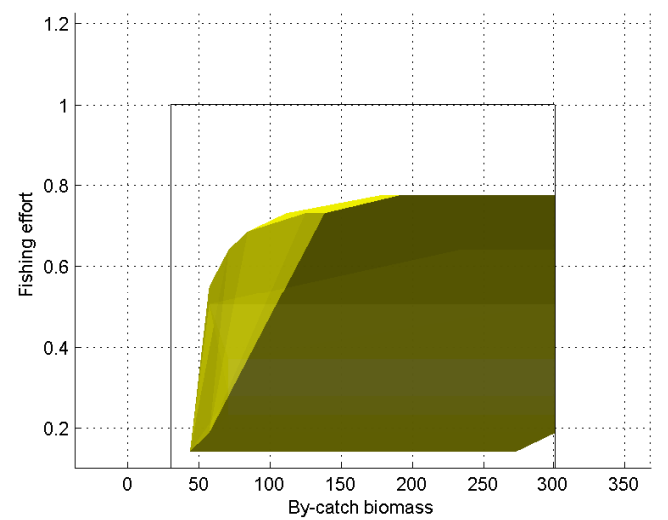

(c) Slice through all values of "target biomass"

Figure 3.4: 2D snapshots of the viability kernel for each of the three dimensions. 
display of the viability space. Consider Figure 3.4a, which displays the kernel in the target biomass vs by-catch biomass space. It is observed that viability requires an implicit minimal biomass for fish stocks, which is larger than their SMBL (i.e. the lower stock boundary). Note that this observation is not apparent in Figure 3.3b, suggesting that the economic constraint imposes a "floor stock" that is greater or more "conservative" than the ecological sustainability criterion defined in (3.9). This is not surprising given that stocks must be sufficiently large to sustain catch rates that guarantee nonnegative cash flows.

From the regulator's point of view, it is important to observe that there is a ceiling on fishing effort that is lower than the maximum fishing capacity $\left(e_{\max }=1\right)$. This is shown in Figures $3.4 \mathrm{~b}$ and $3.4 \mathrm{c}$. Regardless of the state of the fishery's stocks, exerting effort at levels above this ceiling would lead to the over-exploitation of resources. It is also observed in Figure 3.4b that by starting with lower levels of fishing effort, viability of the fishery requires beginning with higher target biomass. A larger target stock is required to ensure that there is sufficient target harvest to satisfy the profitability condition.

Figure 3.5 shows three exemplary evolutions of what happens to the fishery when starting at an initial state $[x(0), y(0), e(0)]$ inside and outside of the kernel. By definition, starting at an initial state inside the kernel, such as $[384,165,0.55] \in \mathcal{V}$, there are smooth strategies (i.e. $u \in[-0.01,0.01]$ ) for which the fishery's evolution remains contained in $\mathcal{V} \in K \subset \mathbb{R}^{3}$. This is confirmed by the solid blue trajectory in Figure 3.5 remaining inside the box. Actually, it remains contained in $\mathcal{V}$. The time profiles of selected variables including the three state variables $(x, y$ and $e$ ) that are associated with 
this trajectory are presented in Figure 3.6. Note that the dynamics of these variables never violate their respective boundaries represented by the solid red lines.

Now consider an initial state in Figure 3.5 where the size of the by-catch biomass is much smaller, such as $[384,57,0.55] \notin \mathcal{V}$. Here, even the fastest fishing effort reduction cannot prevent the by-catch stock from being driven below its SMBL. This is shown by the solid red line eventually leaving the box in finite time via the by-catch biomass lower boundary.

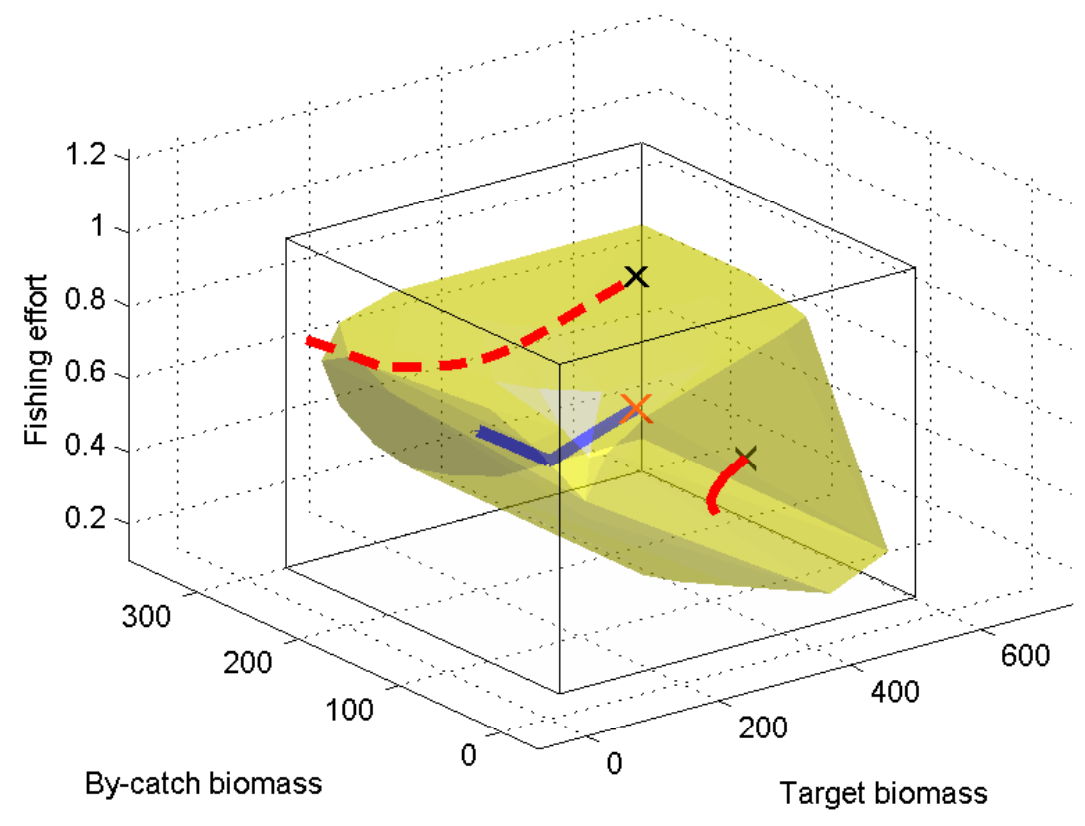

Figure 3.5: Viability kernel with viable and non-viable trajectories.

There is also the case where the target stock can be driven below ecologically sustainable levels. Consider the initial state $[384,165,0.91] \notin \mathcal{V}$, where fishing effort is close to $e_{\max }$ and the by-catch biomass is sufficiently large. 

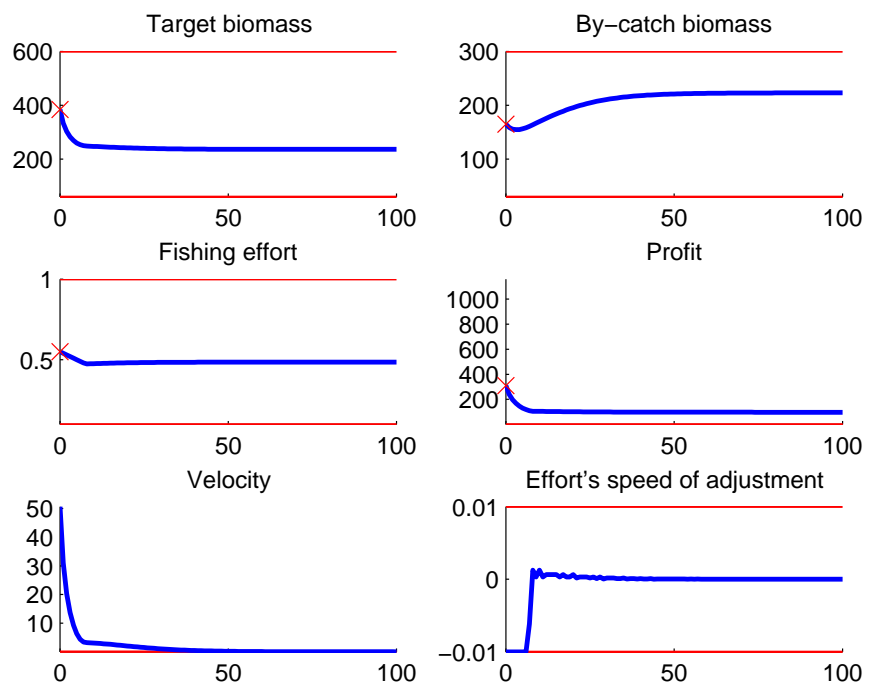

Figure 3.6: Time profiles associated with the viable trajectory displayed in Figure 3.5.

Though the initial target biomass is large, the regulator cannot reduce fishing effort fast enough, given the set of admissible controls available, in order to prevent the target stock from violating its lower boundary. It is noted that as the target biomass nears its SMBL, the profitability condition is expected to be violated (i.e. insufficient target biomass to generate sufficiently large catches). Thus, the fishery may be considered for closure before the target stock hits its lower boundary. It is also noted that as target biomass and consequently target harvest are driven towards zero because of high fishing effort, the by-catch biomass in contrast increases towards its carrying capacity $L_{y}$, see the dash red line in Figure 3.5. This observation is consistent with the bahaviour of the simulated by-catch biomass dynamics noted for high fishing effort in Figure 3.2 (refer back to the relevant discussion). 


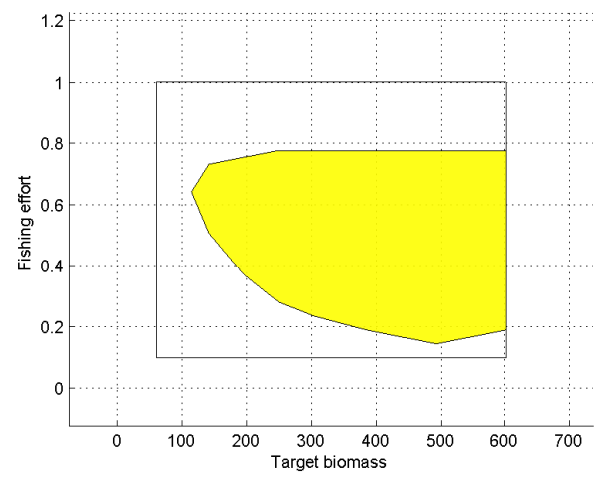

(a) Slice through $y(t)=250$

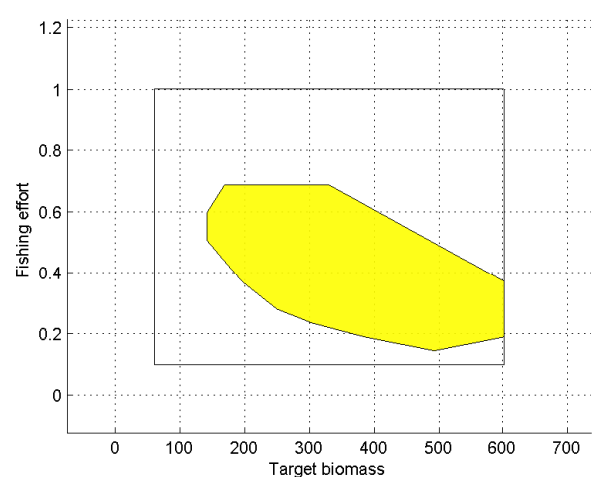

(c) Slice through $y(t)=100$

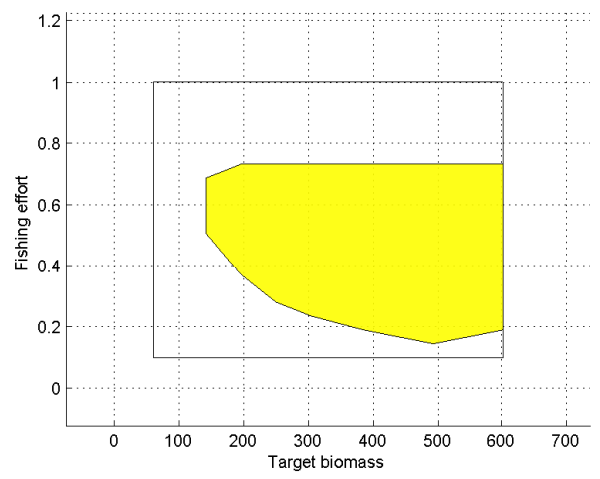

(b) Slice through $y(t)=150$

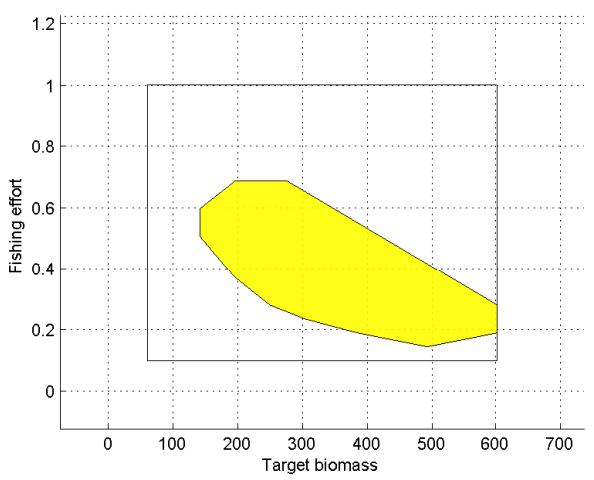

(d) Slice through $y(t)=80$

Figure 3.7: 2D slices of the 3D kernel for different sizes of the by-catch biomass.

Figure 3.7 shows 2D slices of the viability kernel for different starting sizes of the by-catch biomass. There are notably very little changes to the viability kernel (refer to Figures 3.7a and 3.7b) when starting with high levels of bycatch biomass, i.e. at $y(t)=250$ and $y(t)=150$. This is simply because there is no potential risk to the ecological sustainability of the by-catch stock, 
given that the by-catch biomass is sufficiently high ${ }^{10}$. However, significant reductions to the kernel's size are observed for lower starting levels of bycatch biomass, i.e. at $y(t)=100$ and $y(t)=80$, see Figures $3.7 \mathrm{c}$ and $3.7 \mathrm{~d}$. Note that initial states in the upper-right corner (i.e. where levels of $e$ and $x$ are high) of the kernel presented in Figures 3.7a and 3.7b, become non-viable as a result of starting with lower by-catch biomass. This makes sense because with relatively high fishing effort and target biomass, by-catch harvest is also relatively high (refer back to (3.6) and (3.4)), which puts additional pressure on an already sparse by-catch stock.

\subsubsection{A comparison of the single-species and two-species viability kernels}

In Figure 3.8, the two-species viability kernel (Figure 3.3a) is compared with that of the single-species case (Figure 2.3a). The former is a 3D kernel so it is sliced for selected sizes of the by-catch biomass, i.e. at $y(t)=250$ (high by-catch biomass) and $y(t)=100$ (low by-catch biomass), making the two kernels comparable in the same state-space. The shaded (yellow) area in Figures $3.8 \mathrm{a}$ and $3.8 \mathrm{~b}$ represents the $2 \mathrm{D}$ slice of the $3 \mathrm{D}$ kernel for high and low starting values of stock $y$, respectively. The kernel for the single-species model is represented by the scatter plots and displayed in both panels.

The size and shape of the two kernels are similar for the high by-catch biomass case, see Figure 3.8a. By constrast, a significant difference between the two kernels is noted for the low by-catch biomass case, see Figure 3.8b.

\footnotetext{
${ }^{10}$ For the logistic growth model, the level of biomass that supports the maximum sustainable yield is equal to half the carrying capacity, i.e. $y_{\mathrm{MSY}}=\frac{L_{y}}{2}=150$, see (A.9).
} 
It is mostly the area where both fishing effort and target biomass are large that becomes non-viable under the two-species specification. This is not surprising given that with higher $e$ and $x$, there is more pressure placed on the by-catch stock channeled through the corresponding higher by-catch harvest rates. This pressure is simply exacerbated when starting with low bycatch biomass and hence the notable reduction in the size of the two-species kernel.

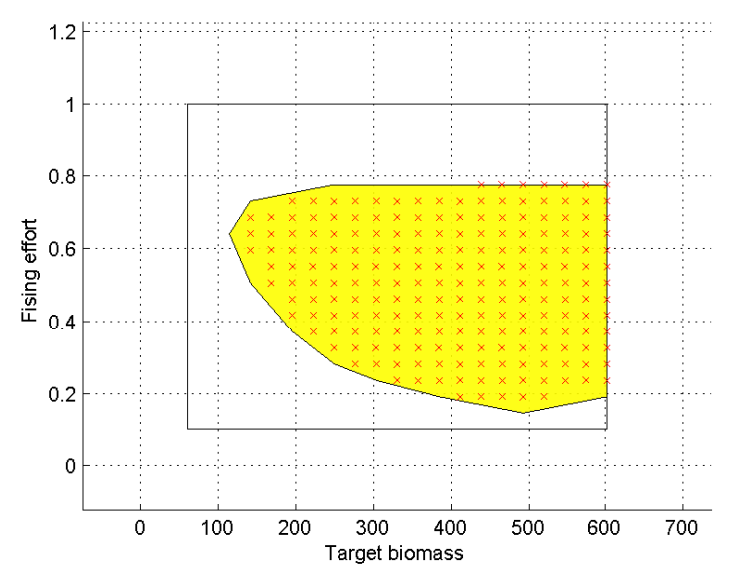

(a) High by-catch biomass

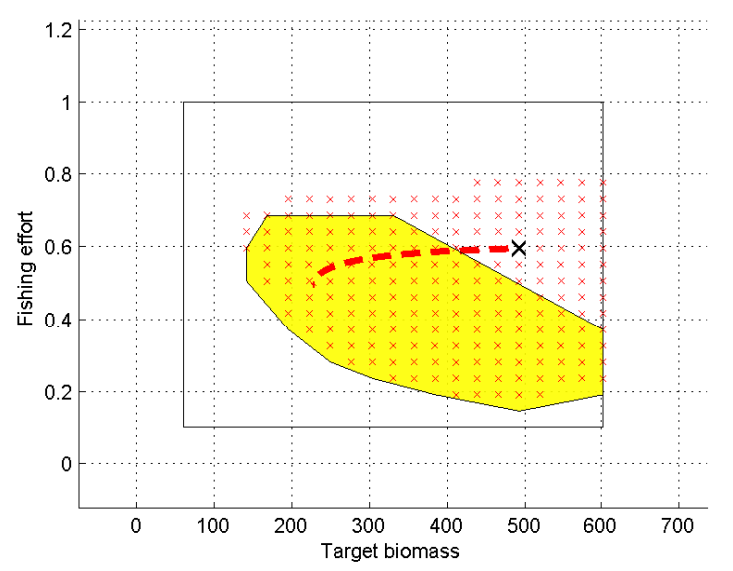

(b) Low by-catch biomass, with an example nonviable trajectory starting from $[492,100,0.595]$

Figure 3.8: A comparison of the two-species and single-species viability kernels for high and low initial values of the by-catch biomass.

In particular, consider an example evolution shown in Figure 3.8b. This evolution begins at the initial state $[492,100,0.595] \notin \mathcal{V}$. The strategy here is to reduce fishing effort as quickly as possible, in an attempt to maintain ecological sustainability. Note that the trajectory is viable under the single-species specification, which is confirmed by the trajectory never leav- 
ing single-species kernel. However, this trajectory is non-viable when the ecological constraint on stock $y$ is imposed. This is because, even with sustained reduction of effort at the maximum allowed rate, the by-catch stock inevitably falls below ecologically sustainable levels, see the panel titled "Bycatch biomass" in Figure 3.9. This implication would not have been observed if the co-dynamics of the jointly harvested fish stocks were over-looked. This shows the inadequacy of the single-species approach to provide reliable forecasting and policy recommendations for sustainable management of multispecies fisheries.
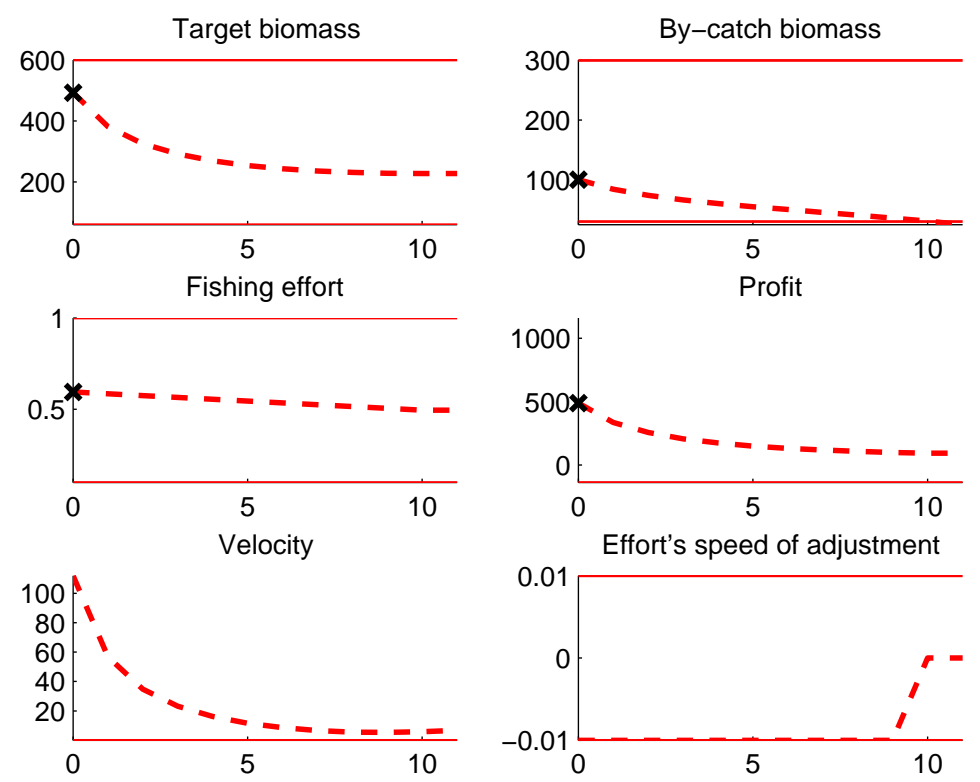

Figure 3.9: Time profiles of selected variables associated with the non-viable trajectory displayed in Figure 3.8b. 


\subsubsection{Sensitivity tests}

Here, the viability kernel for the by-catch problem presented in Figure 3.3a is contrasted with a number of kernels generated for different values of selected parameters. For the purpose of this analysis, the former is herein referred as the base kernel and displayed in yellow in the subsequent figures. In contrast, the kernels generated with the new parameter values are represented in blue.

\section{Lower by-catch growth rate $\left(r_{y}\right)$}

In Figure 3.10, the base kernel is contrasted with that generated for a lower by-catch growth rate, i.e. $r_{y}=0.15$. For a closer look at where these two kernel differ, 2D projections of these kernels are also presented for each of the three dimensions. With a lower $r_{y}$, the size of the kernel is clearly smaller, while its general structure or shape remains unchanged. It is clear from Figures $3.10 \mathrm{~b}$ and $3.10 \mathrm{~d}$ that the lower $r_{y}$ specification induces a higher minimum biomass threshold for the by-catch stock than under the base specification.

\section{Higher by-catch-target harvest ratio $(\alpha)$}

Figure 3.11 contrasts the base kernel with that generated for a higher bycatch-target harvest ratio (i.e. $\alpha=0.3$ ). As in the preceding case, the size of the kernel reduces with a higher value of $\alpha$. Similarly, there is no obvious change in the shape of the kernel with the larger $\alpha$ specification.

\section{Higher fixed cost $(C)$}

Figure 3.12 contrasts the base kernel with that generated with a higher fixed cost, i.e. $C=200$. As in the preceding two scenarios, the size of the kernel 


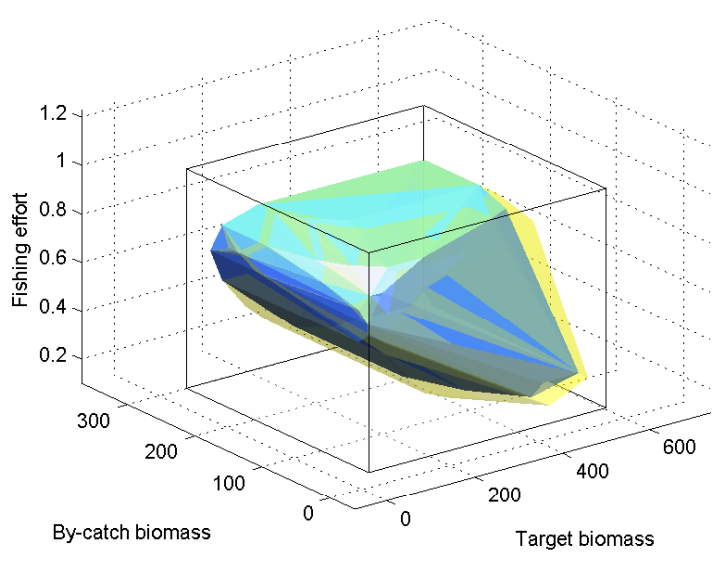

(a) Projection in the 3D space

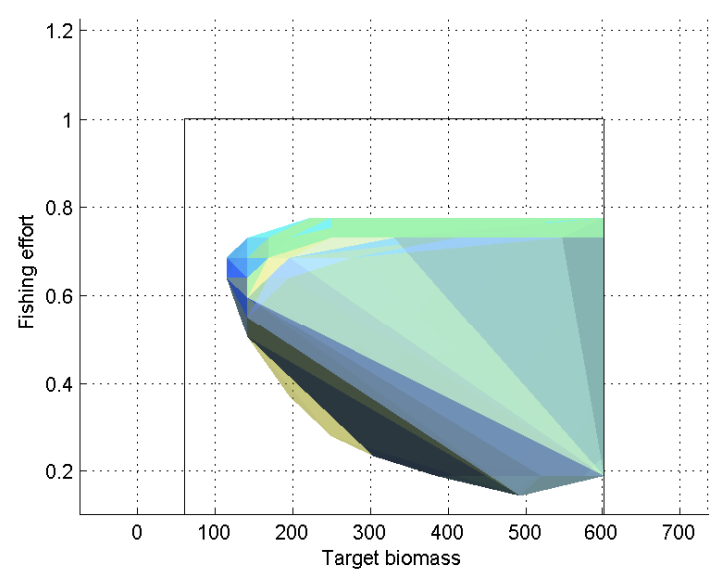

(c) Projection in the $e$ and $x$ space

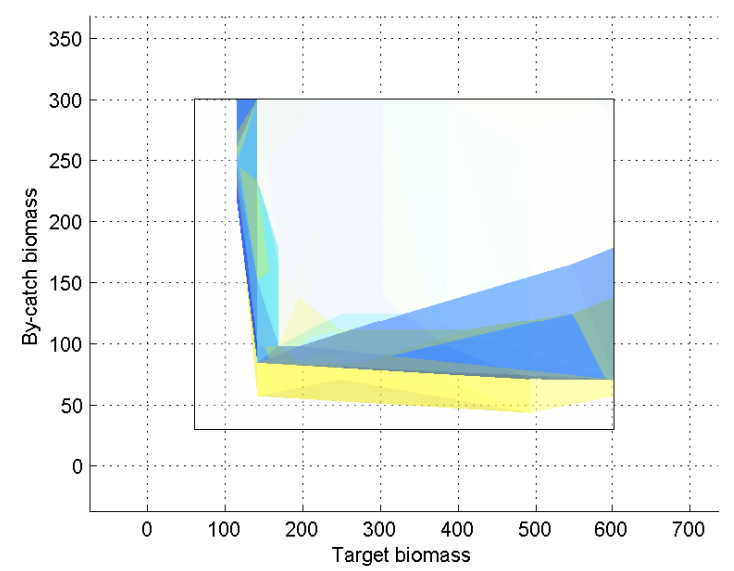

(b) Projection in the $y$ and $x$ space

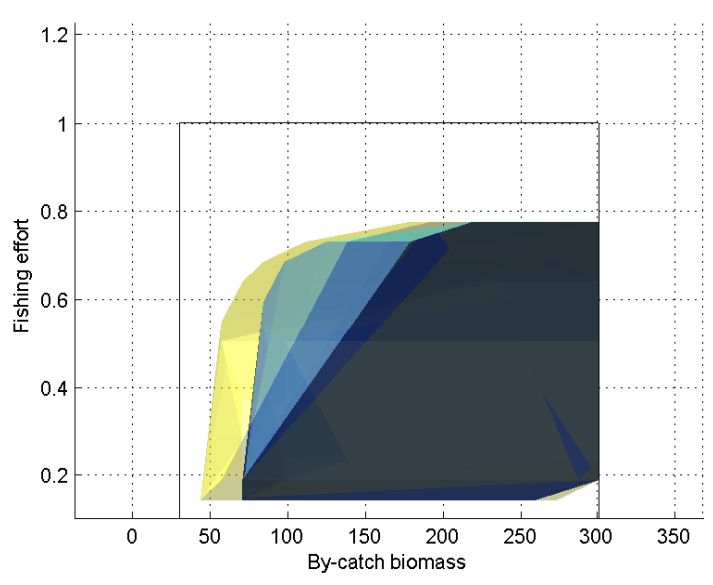

(d) Projection in the $e$ and $y$ space

Figure 3.10: A comparison of the viability kernels generated for $r_{y}=0.2$ and $r_{y}=0.15$. 


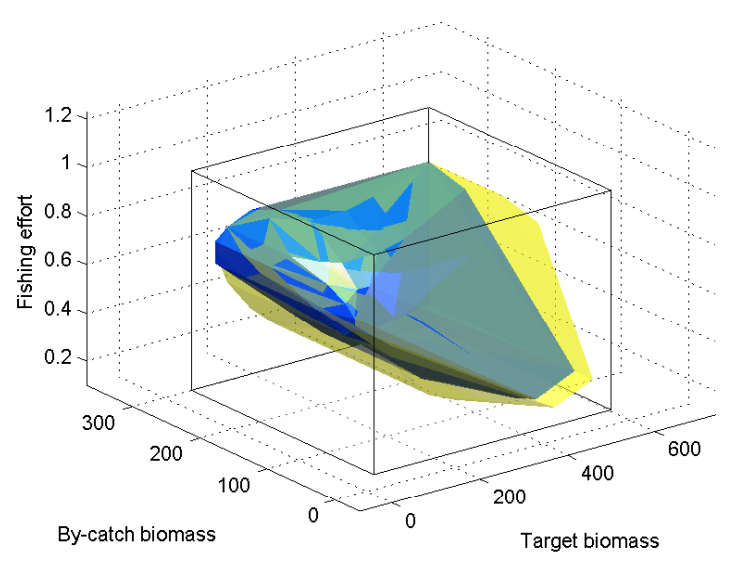

(a) Projection in the 3D space

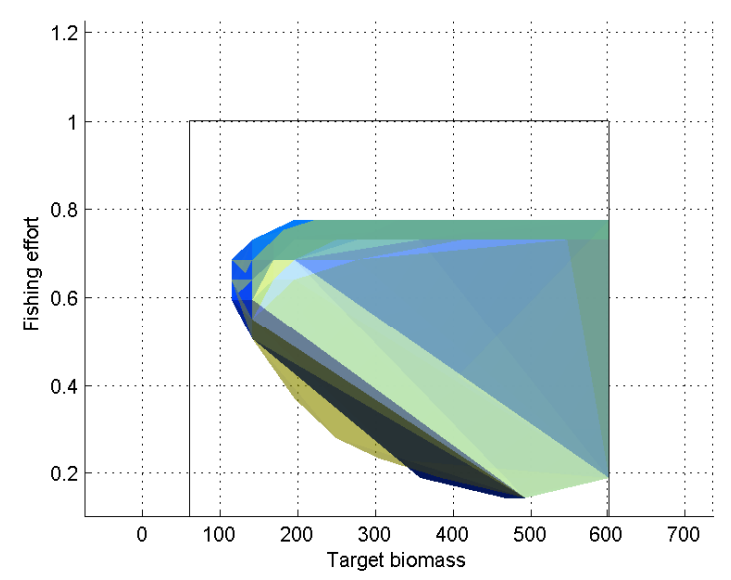

(c) Projection in the $e$ and $x$ space

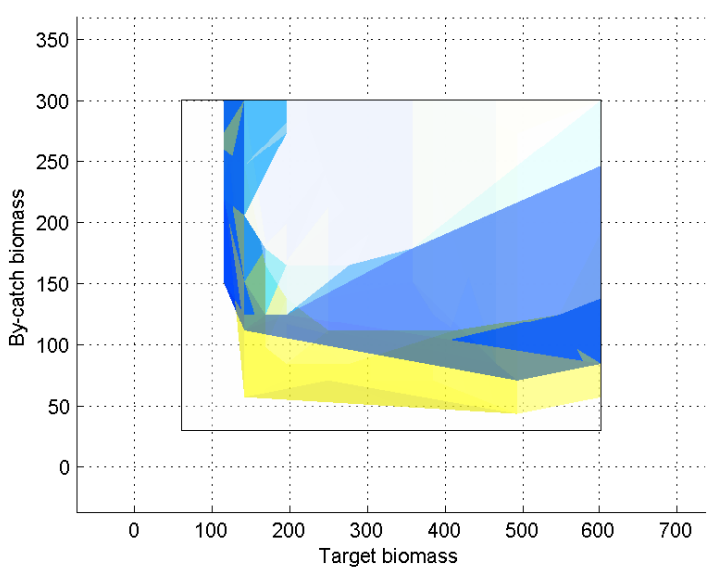

(b) Projection in the $y$ and $x$ space

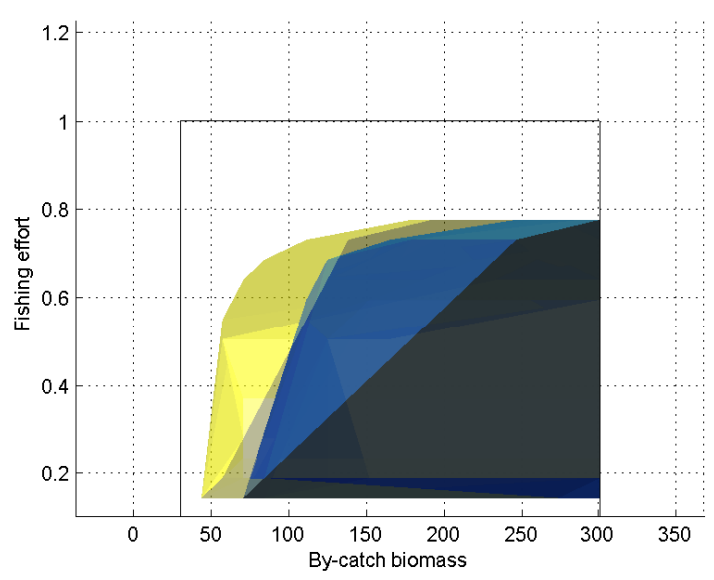

(d) Projection in the $e$ and $y$ space

Figure 3.11: A comparison of the viability kernels generated for $\alpha=0.2$ and $\alpha=0.3$. 


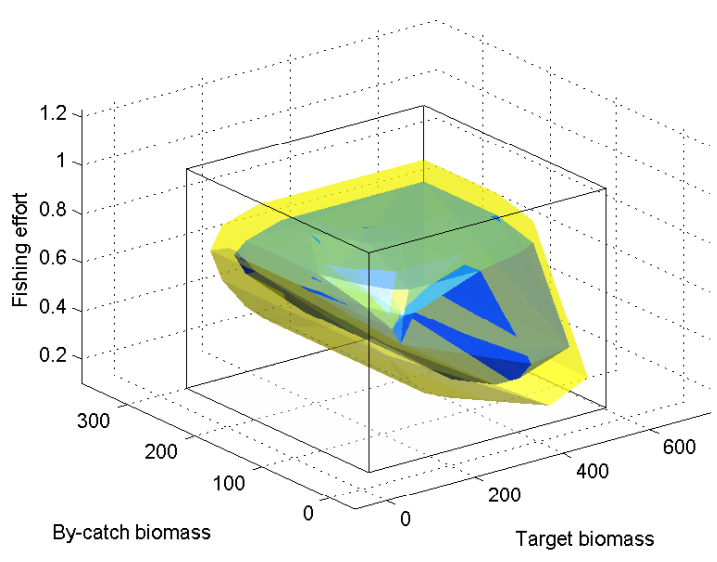

(a) Projection in the 3D space

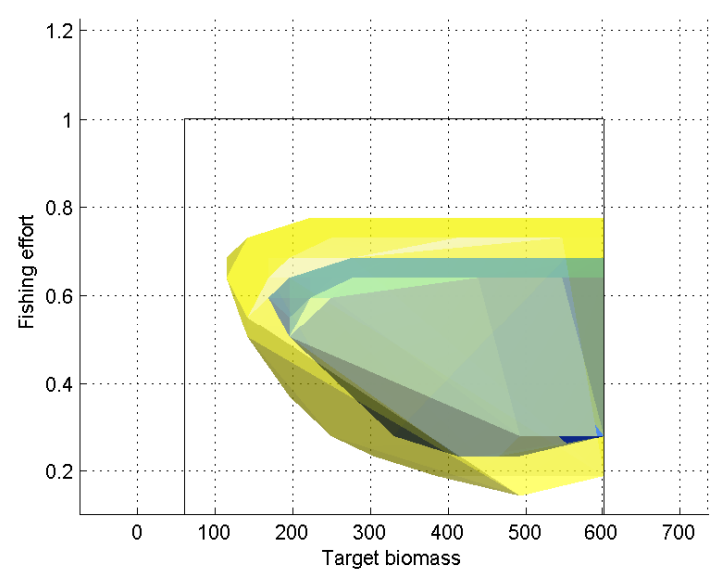

(c) Projection in the $e$ and $x$ space

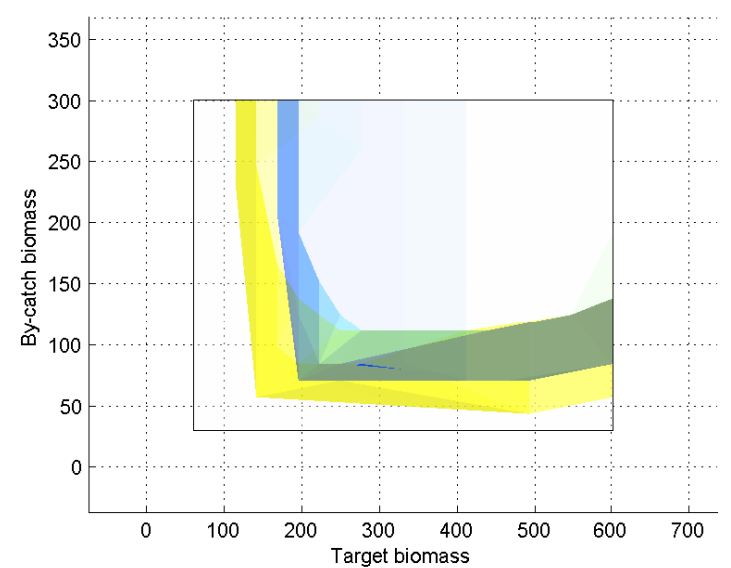

(b) Projection in the $y$ and $x$ space

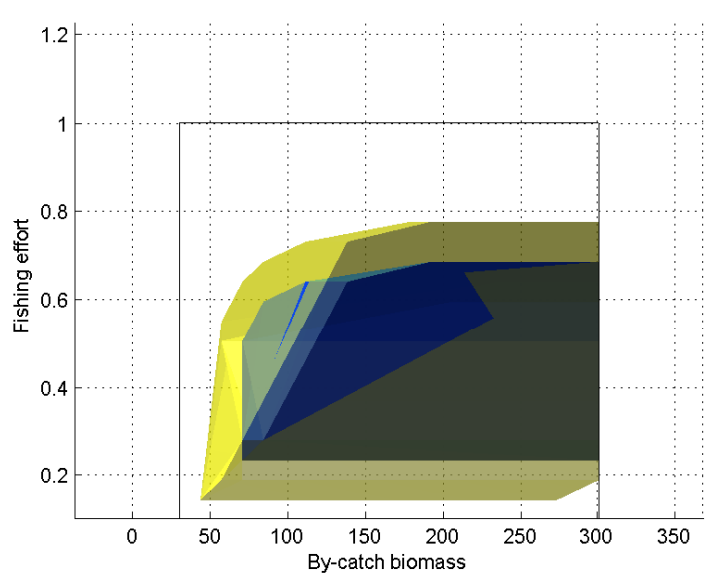

(d) Projection in the $e$ and $y$ space

Figure 3.12: A comparison of the viability kernels generated for $C=150$ and $C=200$. 
reduces with a higher fixed cost. Similarly, there is no obvious change to the shape of the kernel with a higher fixed cost specification.

Thus, from the regulator's perspective, the limited structural change to the kernel noted above for different parameter assumptions is a positive outcome. It implies the robustness of the solutions of the by-catch fishery management problem to parameter uncertainty. As long as the regulator avoids those states that are close to the kernel's boundary, the regulator can be confident that the fishery would not be lead on a catastrophic path even if one (some) of the parameters is (are) misspecified.

\subsubsection{Policy advice}

The viability kernel provides a global picture of how the dynamical system behaves locally in the state-space. From this, the regulator gains an insight of the states from which attainment of the regulator's objectives is guaranteed, and those states that are associated with a catastrophic path leading to the violation of these objectives. From the kernel, the regulator can also infer where in the state-space would the fishery be steered towards given its current position and the proposed policy decision. This may be of value to the regulator for forecasting purposes or evaluating new or existing management targets.

In particular consider Figure 3.13, which shows viable trajectories (in the $e$ and $x$ state-space) for selected points in the viability kernel. By starting anywhere in the kernel, it is known that the system eventually reaches a point on the sustainable equilibrium line, as indicated by the line joining points A and B. Exactly where on this equilibrium line does the regulator like the 
fishery to be in the long-run, depends on the regulator's own prioritisation. So, if the preservation of employment is a priority, then the regulator only has to position the fishery at a state from which a steady state close to point A (where $e$ is high) can be attained. Conservation of the target stock as a priority, requires attaining a steady state that is close to point $\mathrm{B}$ (where $x$ is high). Maximising long-term profit would require reaching a steady state that is the furthest from the zero-profit line (in red), which seems to be half-way between points A and B. Thus, the viability approach establishes satisficing policies or solutions that are generally non-unique and hence amenable to the regulator's own prioritisation without compromising the overall sustainability of the fishery.

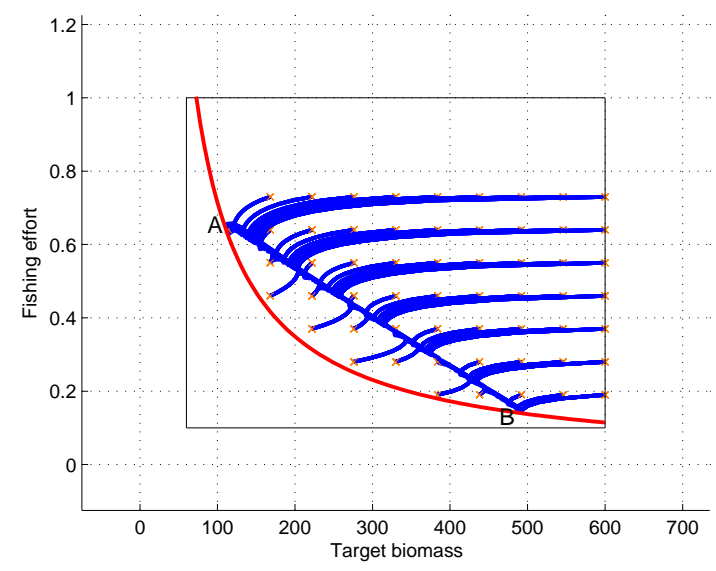

Figure 3.13: Viable paths presented in the fishing effort vs target biomass space.

The regulator can also be confident that these satisficing policies are robust in the face of parameter and model uncertainty. This is a consequence of the precautionary nature of these policies. That is, they are based on the 
system's inertia, making them naturally forward-looking and suitable for any future circumstance (Krawczyk \& Kim, 2009). However, in the case where there exist uncertainty on parameter values, the viability kernel can then be computed using different parameter assumptions. The fishery manager may then choose to maintain the fishery anywhere in the intersection of these kernels. Despite the robustness of these solutions, parameters must still be carefully chosen for accurate and reliable kernel approximations.

The viability analysis presented in this chapter would be of value to a regulator considering moving from a single-species management regime to one that is based on the integrated management of marine resources. One such approach is the ecosystem-based fishery management (EBFM), which is becoming increasingly popular (Arkema et al., 2006). It is a holistic approach that recognises the need to assess and manage the ecological impacts as well as economic and social outcomes related to fishing (Metcalf et al., 2009; Fletcher, 2005). The viability approach is therefore potentially suitable for integrating ecosystem considerations into fisheries management (Cury et al., 2005). There is an existing base of research that has already started doing this, see e.g. Bonneuil (2003); Mullon et al. (2004); De Lara et al. (2012); Doyen et al. (2012); Cissé et al. (2013). 


\subsection{Summary}

This chapter extends the basic model presented in Chapter 2 to account for the dynamics of a by-catch fish stock, which is assumed to be incidentally caught as a result of harvesting a targeted fish stock. The focus of the analysis is to establish the configurations required to guarantee the survival of the by-catch stock, while maintaining the ecological sustainability of the target stock and sustaining fishing activity and profitability of the fishery. To achieve this, the viability kernel for the by-catch problem is generated using VIKAASA. It is shown that under the multi-species specification, there are fewer combinations of fishing effort and target biomass that are viable relative to the single-specifies specification. These changes are noticeable for lower sizes of the by-catch stock. It is those combinations of relatively high fishing effort and target biomass that become non-viable when the co-dynamics of jointly harvested fish stocks are specified.

Although the model developed in this chapter is quite stylised, it is shown that the viability kernel is a useful qualitative tool for a regulator tasked with addressing the by-catch problem. Specifically, the qualitative analysis is successful in investigating the system stability and the impacts of parameter uncertainty on the behaviour of the system, and in highlighting the likely viable and non-viable states.

In contrast to the current chapter, which assumes a fishery regulated by an input (or fishing effort) control, the next chapter casts the by-catch problem in the context of an output control based management regime that imposes restrictions on the amount of fish coming out of the fishery. The quantity or output measure is supplemented with a price instrument that aims to 
provide economic incentives to economic agents to restrict their landings below allowable levels. 


\section{Chapter 4}

\section{A viability analysis of output-controlled fisheries}

\subsection{An overview}

The previous chapter considered a by-catch fishery where the regulator manages fishing activity at sustainable levels by controlling fishing effort adjustments and hence the intensity of fishing methods used to catch fish. This chapter, by contrast, considers an output-controlled management regime based on New Zealand's Quota Management System (QMS), which imposes direct control over harvest levels for each fish species.

Since its inception in 1986, the QMS has undergone major administrative and legislative changes ${ }^{1}$. Some of these developments are briefly described below. For a more complete account of the history and institutional detail of

\footnotetext{
${ }^{1}$ For instance, the introduction of the deemed value system in 2001 shifted the QMS from a criminal offence-based regime to an administrative regime based on economic incentives (Peacey, 2002).
} 
the QMS, see e.g. Lock \& Leslie (2007); Mace et al. (2013) and the references therein.

In 2010, there were 636 fish stocks spread over 100 species or species groupings covered by the QMS (Mace et al., 2013)2. Initially, 161 fish stocks for 28 species were managed by the QMS at its inception in 1986 (Peacey, 2002; Walker \& Townsend, 2008). In order to maintain more control over the sustainable utilisation of fisheries resources, each species subject to the QMS is divided into separate fish stocks, which are managed independently in a nominated geographical area, officially designated as the quota management area (QMA) . A fish species can consist of numerous geographically isolated and biologically distinct populations, making it rather difficult to manage each species at the national level (Lock \& Leslie, 2007). The QMA boundaries for each species is determined primarily by the fisheries management areas (FMAs) that define New Zealand's exclusive economic zone, see Figure 4.1. Depending on biological and administrative factors, a QMA may be an amalgamation of more FMAs.

The Minister for Primary Industries sets an annual total allowable catch (TAC) for each fish stock after considering biological assessments and other sources of information ${ }^{3}$. The TAC is set with the goal of maintaining the fish population at or above a level that can sustain the largest possible yield, i.e. MSY. From the TAC, an allowance is made to non-commercial fishing (i.e. recreational fishing and customary uses). The remainder is allocated to the commercial sector as the total allowable commercial catch (TACC).

\footnotetext{
${ }^{2}$ Currently, there are 638 QMS fish stocks (Ministry for Primary Industries, 2013).

${ }^{3}$ The former Ministry of Fisheries has merged with the Ministry of Agriculture and Forestry and the New Zealand Food Safety Authority to form the Ministry for Primary Industries.
} 


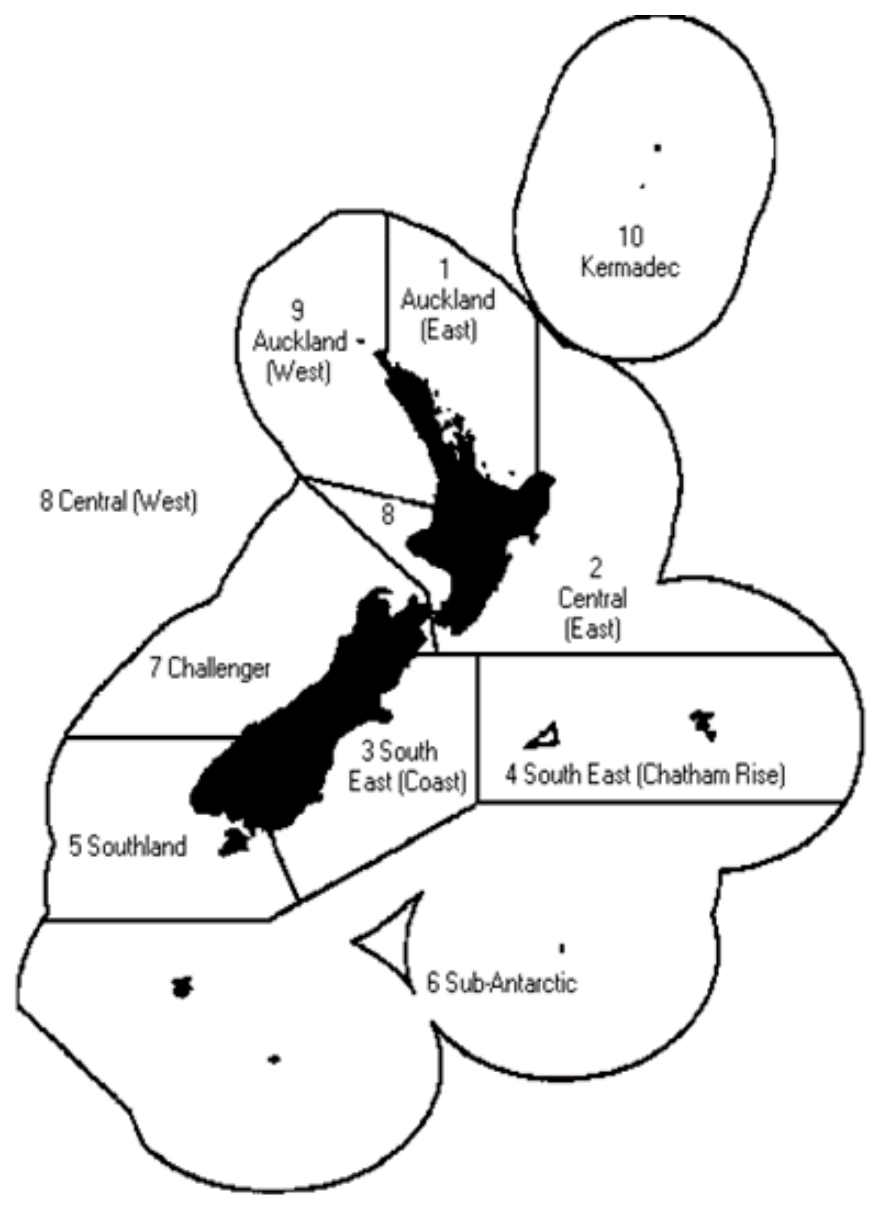

Figure 4.1: Fisheries Management Areas. Source: Ministry for Primary Industries (2005). 
The TACC is divided into individual transferable quotas (ITQs) that are, in effect, the in-perpetuity right to receive a share of the TACC each year. Since 2001, at the start of each fishing year, a quota owner's ITQ generates an annual catch entitlement (ACE), which is the tonnage equivalent of the quota share of the commercial catch limit. ACE holders can use it to cover catch or sell it at the going market ACE price to other agents who have insufficient or no ACE. The objective is to ensure that the TACC is not over-caught by encouraging agents to balance all of their catch (i.e. both target and by-catch fish stocks) with ACE. This is achieved by supplementing the QMS with a penalty-like mechanism, known as the deemed value system (DVS). The DVS allows agents to land catch that is not covered by ACE through payment of a deemed value on each unit of catch in excess of ACE holdings. The deemed value is set sufficiently high such that any financial benefit from landing the catch without ACE is eliminated and reducing incentives to catch stocks for which agents have insufficient or no ACE, and low enough so that agents have an incentive to land rather than discard any uncovered catch. It is assumed in this study that the deemed value is set appropriately, so agents are assumed not to engage in the practice of dumping or discarding, i.e. what is caught is also landed.

This thesis is mainly concerned with the sustainable utilisation of fisheries resources by the commercial sector, so the focus herein is on modelling the system wide implications (i.e. ecological, economic and social) of TACC policies. For convenience, it is assumed that all of the TAC is allocated to commercial fishing, i.e. TAC $=$ TACC. Moreover, the aim of the present chapter is not to provide a full account of the QMS, but to highlight important elements of the QMS on which to base a viability model (referred 
henceforth as the output-controlled fisheries model ${ }^{4}$ ) that reflects, as much as possible, New Zealand's fisheries management regime. Using this model, an attempt is made to address the by-catch problem.

This chapter is organised as follows. The viability model is developed in Section 4.2. This includes outlining key features of the QMS to be incorporated into the viability model, specifying the proposed cost and profit functions, deriving the ACE price, and defining the system dynamics and viability constraints. For convenience, the mathematical symbols and their descriptions for the model developed here are available from the List of Symbols. The model is calibrated in Section 4.3. An analysis of the viability kernel approximated by VIKAASA for the output-controlled fisheries model is presented in Section 4.4. Some policy advice is also provided before wrapping up this chapter with some concluding remarks in Section 4.5.

\subsection{A viability model}

\subsubsection{Outlining the Quota Management System}

Following Herrera (2005), the system considered here consists of two ecologically independent fish stocks with fishable biomasses $x(t)$ and $y(t)$, which are targeted by two groups of harvesters or fisheries denoted by $F^{B}$ and $F^{T}$, respectively. The superscript $B$ stands for the by-catch fishery and $T$ stands for the target fishery. Suppose that the system is characterised by a "unilateral by-catch process" where agents in $F^{B}$ target and catch stock $x$, and incidently catch stock $y$. Agents in $F^{T}$ target and only catch stock $y$. To

\footnotetext{
${ }^{4}$ Here, two fisheries are considered as opposed to only one fishery in the previous chapter.
} 
distinguish an agent in $F^{B}$ from an agent in $F^{T}, i$ and $j$ are used to denote an individual agent in $F^{B}$ and $F^{T}$, respectively.

At the beginning of each fishing period $t$, the regulator sets TACCs for fish stocks $x$ and $y$ denoted by $A_{x}(t)$ and $A_{y}(t)$, respectively. The regulator allocates $A_{x}(t)$ among active agents in $F^{B}$ and $A_{y}(t)$ among those active in $F^{T}$. An agent's share of the TACC is assumed here to generate all the ACE (denominated in tonnes) against which the agent balances his catch for that fishing period ${ }^{5}$. It is assumed that agents in both fisheries are free to trade all or a portion of their ACE. However, inter-temporal trade of ACE is not allowed ${ }^{6}$.

Let $a_{i x}(t)$ denote agent $i$ 's ACE holding for stock $x$ after trade in period $t$. Similarly, let $a_{j y}(t)$ denote agent $j$ 's ACE holding for stock $y$ after trade in period $t$. It is assumed that there is a linear relationship between an agent's catch of the stock targeted and his ACE holding for that stock. That is,

$$
\begin{aligned}
& h_{i x}(t)=\beta_{x} a_{i x}(t) \quad \forall i=1,2, \ldots, n^{B} \\
& h_{j y}(t)=\beta_{y}^{T} a_{j y}(t) \quad \forall j=1,2, \ldots, n^{T},
\end{aligned}
$$

where the parameters $\beta_{x}$ and $\beta_{y}^{T}$ are indicators of whether (on average) agents' catch differs from their ACE and in which direction ${ }^{7}$. So $\beta_{x}<1$ and $\beta_{y}^{T}<1\left(\beta_{x}>1\right.$ and $\left.\beta_{y}^{T}>1\right)$ imply that agents in $F^{B}$ and $F^{T}$ under-catch

\footnotetext{
${ }^{5}$ In fact, quota at time $t$ generates most of the current fishing year's ACE as quota right owners in New Zealand are permitted to carry forward up to $10 \%$ of unbalanced ACE from the previous fishing year (Peacey, 2002). This, however, does not apply to fish stocks listed under schedule 5A of the Fisheries Act 1996.

${ }^{6}$ This assumption may not hold true in reality given that ACE holders can carry forward up to $10 \%$ of unbalanced ACE.

${ }^{7}$ Note that the superscript $B$ is dropped from $\beta_{x}$ because agents in $F^{T}$ do not hold ACE for stock $x$.
} 
(over-catch) their ACE. Agents just-catch their ACE when $\beta_{x}=\beta_{y}^{T}=1$. The number of active agents in $F^{B}$ and $F^{T}$ are given by $n^{B}$ and $n^{T}$, respectively.

This chapter is interested in investigating the aggregate effect of under- or over-fishing the TACCs on the viability of the system. Individual behaviours are therefore irrelevant. Given this, it is assumed that agents in both fisheries are homogenous in their decision to under- or over-catch their ACE. This is expected to be true because all agents face the same economic conditions.

As mentioned earlier, agents in the by-catch fishery jointly harvest fish stocks $x$ and $y$, where the latter is considered to be the by-catch of targeting the former. Suppose that the joint production function takes the linear form,

$$
h_{i y}(t)=\alpha h_{i x}(t)
$$

where $h_{i x}(t)$ is defined by (4.1). The parameter $\alpha$ denotes the by-catchtarget harvest ratio, which is a measure of the intensity of jointness of the production relationship.

Note that agents in $F^{B}$ are also required to cover their catch of stock $y$ with ACE. They purchase ACE for stock $y$ from agents in $F^{T}$. Similarly, a linear relationship between agent $i$ 's catch of stock $y$ and his ACE holding for that stock is proposed,

$$
h_{i y}(t)=\beta_{y}^{B} a_{i y}(t) \quad \forall i=1,2, \ldots, n^{B},
$$

where $a_{i y}(t)$ denotes agent $i$ 's ACE holding for stock $y$ in period $t$ and $\beta_{y}^{B}$ is a measure of the propensity that agent $i$ under-catches $\left(\beta_{y}^{B}<1\right)$, just-catches $\left(\beta_{y}^{B}=1\right)$ or over-catches $\left(\beta_{y}^{B}>1\right)$ his ACE for stock $y$. Using (4.1), (4.3) and (4.4), $\beta_{y}^{B}$ can be obtained,

$$
\beta_{y}^{B}=\frac{\alpha \beta_{x} a_{i x}(t)}{a_{i y}(t)},
$$


which is simply the ratio between agent $i$ 's catch of stock $y$ and his ACE holding for that stock.

\section{ACE and deemed value payments}

An agent's catch must be covered with ACE, otherwise the agent is liable to pay the deemed value on the volume of uncovered catch. As mentioned earlier, agents are allocated a share of the TACC at the beginning of each fishing year, which generates ACE for that period. So agents, in fact, do not have to purchase ACE for catch already covered by their annual allocation of $\mathrm{ACE}^{8}$. Despite this, there is an opportunity cost of fishing the allocated $\mathrm{ACE}$, i.e. the return from selling some or all of the allocated ACE at the going price. Here, this opportunity cost is taken into consideration when modeling agents' costs.

Thus, agents pay the stock's ACE price for every unit of catch landed or until his ACE holding is exhausted. Let $m_{x}(t)$ and $m_{y}(t)$ denote the ACE price for stocks $x$ and $y$, respectively. These ACE prices are determined in Section 4.2.2.

Agent $i$ 's payment of ACE for stock $x$ is as follows ${ }^{9}$,

$$
\begin{aligned}
\Lambda_{i x}(t) & =\left\{\begin{array}{l}
m_{x}(t) h_{i x}(t) \quad \text { for } \beta_{x}<1 \\
m_{x}(t) a_{i x}(t) \quad \text { for } \beta_{x} \geq 1
\end{array}\right. \\
& =m_{x}(t) \min \left[h_{i x}(t), a_{i x}(t)\right] .
\end{aligned}
$$

\footnotetext{
${ }^{8}$ Note that agents in $F^{B}$ are not allocated ACE for stock $y$. It is assumed that they do not hold quota for stock $y$ and therefore have to purchase ACE from agents in $F^{T}$.

${ }^{9}$ The min function is used so that the piecewise function can be collapsed into a singleline equation. This is because only the latter can be specified in VIKAASA.
} 
Agent $i$ 's payment of ACE for stock $y$ is as follows,

$$
\begin{aligned}
\Lambda_{i y}(t) & = \begin{cases}m_{y}(t) h_{i y}(t) & \text { for } \beta_{y}^{B}<1 \\
m_{y}(t) a_{i y}(t) & \text { for } \beta_{y}^{B} \geq 1\end{cases} \\
& =m_{y}(t) \min \left[h_{i y}(t), a_{i y}(t)\right]
\end{aligned}
$$

Agent $j$ 's payment of ACE for stock $y$ is as follows,

$$
\begin{aligned}
\Lambda_{j y}(t) & = \begin{cases}m_{y}(t) h_{j y}(t) & \text { for } \beta_{y}^{T}<1 \\
m_{y}(t) a_{j y}(t) & \text { for } \beta_{y}^{T} \geq 1\end{cases} \\
& =m_{y}(t) \min \left[h_{j y}(t), a_{j y}(t)\right]
\end{aligned}
$$

Additionally, agents are liable to pay the (base) deemed values, denoted by $d_{x}$ and $d_{y}$, for every unit of catch in excess of their ACE holdings for stocks $x$ and $y$, respectively. The deemed value is set by the regulator and it is assumed here to be fixed for both stocks. It is set above the ACE price, which gives an incentive to agents to balance their catch with $\mathrm{ACE}$, but lower than the landing price to discourage illegal discarding ${ }^{10}$. So, theoretically the deemed value is an upper bound on the ACE price, which acts on catch vs ACE similarly to a backstop technology as defined in Hotelling (1931).

\footnotetext{
${ }^{10}$ Some fish stocks in New Zealand have prohibitive deemed value, which makes it uneconomic to catch beyond self ACE holding. Additionally, some stocks have differential deemed values. For example, for levels of catch $h$ in excess of ACE $a$ of up to 20\%, the basic annual deemed value, $d$ is charged. For levels of catch in excess of ACE between $20 \%$ and $100 \%$, annual deemed value is charged at an increasing proportion of the basic rate, i.e. $d\left(1+\frac{h-a}{a}\right)$ until the catch is twice the ACE owned when the deemed value charged is twice the basic annual deemed value, i.e. $2 \times d$ (Peacey, 2002). For simplicity the fish stocks considered here are not subject to differential deemed values.
} 
Agent $i$ 's deemed value payment for stock $x$ is as follows ${ }^{11}$,

$$
\begin{aligned}
\Omega_{i x}(t) & = \begin{cases}0 & \text { for } \beta_{x} \leq 1 \\
d_{x}\left(h_{i x}(t)-a_{i x}(t)\right) & \text { for } \beta_{x}>1\end{cases} \\
& =d_{x} \max \left[0, h_{i x}(t)-a_{i x}(t)\right] .
\end{aligned}
$$

Agent $i$ 's deemed value payment for stock $y$ is as follows,

$$
\begin{aligned}
\Omega_{i y}(t) & = \begin{cases}0 & \text { for } \beta_{y}^{B} \leq 1 \\
d_{y}\left(h_{i y}(t)-a_{i y}(t)\right) & \text { for } \beta_{y}^{B}>1\end{cases} \\
& =d_{y} \max \left[0, h_{i y}(t)-a_{i y}(t)\right] .
\end{aligned}
$$

Agent $j$ 's deemed value payment for stock $y$ is as follows,

$$
\begin{aligned}
\Omega_{j y}(t) & = \begin{cases}0 & \text { for } \beta_{y}^{T} \leq 1 \\
d_{y}\left(h_{j y}(t)-a_{j y}(t)\right) & \text { for } \beta_{y}^{T}>1\end{cases} \\
& =d_{y} \max \left[0, h_{j y}(t)-a_{j y}(t)\right] .
\end{aligned}
$$

\section{Production costs}

Furthermore, the fishing activity incurs production costs that depend positively on harvest and negatively on the biomass of the stock being targeted. The cost function is assumed to be smooth enough $\left(\mathcal{C}^{2}\right)$ on $\mathbb{R}_{+}$and strictly convex with respect to harvest ${ }^{12}$. The latter implies that marginal cost of

\footnotetext{
${ }^{11}$ Similarly, the max function is used so that the piecewise function simplifies into a single-line equation.

${ }^{12}$ The convexity property is widely accepted and used in the fisheries economics literature. In an earlier paper, Doll (1988) provides a detailed review of the vessel cost functions and related short-run concepts as presented in the conventional literature of fishery economics.
} 
harvest increases with the level of harvest. As more and more agents compete for a fixed stock of fish, at least in the short-run, it becomes progressively harder for agents to maintain their catch rate because of the scarcity of fish, so agents are forced to spend more time searching and fishing, and pushing costs upwards in the process. Additionally, as the vessel or gear is used more frequently during the fishing period, total variable costs will rise slowly and then more rapidly as full utilisation requires more maintenance, replacement of gears, and the like.

It is proposed that agent $i$ 's cost of production comprises two parts: a separable and a non-separable component. The former implies that the agent incurs a cost that is associated with harvesting a particular stock, e.g. handling, cleaning, preserving, etc. It is assumed that the separable cost is linear in harvest. Non-separable cost includes all variable costs that cannot be independently attributed to harvesting a particular stock or simply the cost attributed to catching the stocks jointly, e.g. the cost of winching the nets out and in, gear maintenance and repair, search cost, fuel, etc. It is assumed that the non-separable cost is non-linear, increasing in harvest and decreasing in the targeted stock's biomass. Similarly, agent $j$ 's production cost consists of a linear and non-linear part.

Agents may also incur fixed costs, e.g. yearly lease of a vessel from its owner, insurance, taxes, depreciation and the appropriate opportunity costs (other than the forgone return from selling their allocated ACE). However, these fixed costs are not accounted for in the cost function specified for the output-controlled fisheries model, simply because the analysis is concerned with the marginal behaviour of agents ${ }^{13}$.

${ }^{13}$ Other reasons include (1) a fixed cost would not add anything other than an upward 
Agent $i$ 's production cost function reads as follows,

$$
P C_{i}(t)=c_{x} h_{i x}(t)+c_{y} h_{i y}(t)+\frac{w^{B}}{2 x(t)}\left(h_{i x}(t)+h_{i y}(t)\right)^{2},
$$

where $c_{x}$ and $c_{y}$ are fixed unit costs that can be independently attributed to harvesting stocks $x$ and $y$, respectively. The parameter $w^{B}$ is a cost associated with the non-separable cost of harvesting both stocks jointly.

Agent $j$ 's production cost function reads as follows,

$$
P C_{j}(t)=c_{y} h_{j y}(t)+\frac{w^{T}}{2 y(t)}\left(h_{j y}(t)\right)^{2} .
$$

It is assumed that agents face the same fixed unit cost $c_{y}$ of harvesting stock $y$ in both fisheries. Given that this cost is associated with the activity that follows from landing the catch, it is reasonable to expect that the cost of such activity is not significantly different across fisheries for a particular stock. For instance, the hourly labour rate charged for handling and cleaning stock $y$ in $F^{T}$ should not be much different from that charged for the same activity on the same stock in another fishery. The parameter $w^{T}$ denotes the cost associated with the non-linear cost of harvest in the target fishery. Obviously, with no by-catch there is no reason to expect that agents in $F^{T}$ have an identical production technology to that employed in $F^{B}$.

\footnotetext{
shift in the cost function, (2) the vessel and gears could be used in other fisheries so the investment could be shared among several fishing activities, and (3) the fixed cost simply disappears when deriving the ACE prices so it add an unnecessary constant to an already complex mathematical notation.
} 


\section{Profit}

Agents in both fisheries are considered to be price takers in the output market. They face a common exogenous unit price $p_{x}$ and $p_{y}$ for fish stocks $x$ and $y$, respectively.

The short form of agent $i$ 's profit function is defined as,

$$
\pi_{i}(t)=p_{x} h_{i x}(t)+p_{y} h_{i y}(t)-P C_{i}(t)-\Lambda_{i x}(t)-\Lambda_{i y}(t)-\Omega_{i x}(t)-\Omega_{i y}(t),
$$

where $P C_{i}(t), \Lambda_{i x}(t), \Lambda_{i y}(t), \Omega_{i x}(t)$ and $\Omega_{i y}(t)$ are defined in (4.12), (4.6), (4.7), (4.9) and (4.10), respectively.

The short form of agent $j$ 's profit function is defined as,

$$
\pi_{j}(t)=p_{y} h_{j y}(t)-P C_{j}(t)-\Lambda_{j y}(t)-\Omega_{j y}(t)
$$

where $P C_{j}(t), \Lambda_{j y}(t)$ and $\Omega_{j y}(t)$ are defined in (4.13), (4.8) and (4.11), respectively.

In what follows the ACE price is derived for each fish stock.

\subsubsection{Deriving the ACE price}

The ACE prices for stocks $x$ and $y$ are derived using the method described in Péreau et al. $(2012)^{14}$. To maintain the readability of this thesis, this section is limited to the derivation of the ACE price for stock $x$ only. The ACE price for stock $y$ is derived in Section C.1 of the Appendix to this chapter. Note that the time argument on all dynamic variables is dropped. This unburdens the notation.

\footnotetext{
${ }^{14}$ Péreau et al. (2012) deal with a single-species fishery. They also assume that catches do not deviate from catch entitlements, i.e. quota.
} 
By substituting (4.6), (4.7), (4.9), (4.10) and (4.12) into (4.14), the long form of agent $i$ 's profit function is obtained,

$$
\begin{aligned}
\pi_{i} & =p_{x} h_{i x}+p_{y} h_{i y} \\
& -c_{x} h_{i x}-c_{y} h_{i y}-\frac{w^{B}}{2 x}\left(h_{i x}+h_{i y}\right)^{2} \\
& -m_{x} \min \left[h_{i x}, a_{i x}\right]-m_{y} \min \left[h_{i y}, a_{i y}\right] \\
& -d_{x} \max \left[0, h_{i x}-a_{i x}\right]-d_{y} \max \left[0, h_{i y}-a_{i y}\right]
\end{aligned}
$$

Using (4.1), (4.3) and (4.4), agent $i$ 's profit function (4.16) is expressed as a function of $h_{i x}$,

$$
\begin{aligned}
\pi_{i} & =\left(p_{x}-c_{x}\right) h_{i x}+\alpha\left(p_{y}-c_{y}\right) h_{i x}-\frac{w^{B}}{2 x}\left((1+\alpha) h_{i x}\right)^{2} \\
& -\left(m_{x} \min \left[1, \frac{1}{\beta_{x}}\right]+\alpha m_{y} \min \left[1, \frac{1}{\beta_{y}^{B}}\right]\right) h_{i x} \\
& -\left(d_{x} \max \left[0,1-\frac{1}{\beta_{x}}\right]+\alpha d_{y} \max \left[0,1-\frac{1}{\beta_{y}^{B}}\right]\right) h_{i x} .
\end{aligned}
$$

Differentiating (4.17) with respect to $h_{i x}$,

$$
\begin{aligned}
\frac{\partial \pi_{i}}{\partial h_{i x}} & =p_{x}-c_{x}+\alpha\left(p_{y}-c_{y}\right)-\frac{w^{B}(1+\alpha)^{2}}{x} h_{i x} \\
& -m_{x} \min \left[1, \frac{1}{\beta_{x}}\right]-\alpha m_{y} \min \left[1, \frac{1}{\beta_{y}^{B}}\right] \\
& -d_{x} \max \left[0,1-\frac{1}{\beta_{x}}\right]-\alpha d_{y} \max \left[0,1-\frac{1}{\beta_{y}^{B}}\right]=0
\end{aligned}
$$

and solving for $h_{i x}$ yields,

$$
\begin{aligned}
h_{i x}^{*} & =\frac{x}{w^{B}(1+\alpha)^{2}}\left\{p_{x}-c_{x}-m_{x} \min \left[1, \frac{1}{\beta_{x}}\right]-d_{x} \max \left[0,1-\frac{1}{\beta_{x}}\right]\right. \\
& \left.+\alpha\left(p_{y}-c_{y}-m_{y} \min \left[1, \frac{1}{\beta_{y}^{B}}\right]-d_{y} \max \left[0,1-\frac{1}{\beta_{y}^{B}}\right]\right)\right\} .
\end{aligned}
$$


Aggregating (4.18) across all $n^{B}$ agents yields the demand for ACE,

$$
\begin{aligned}
H_{x}^{*} & =\sum_{i=1}^{n^{B}} h_{i x}^{*} \\
& =n^{B} h_{i x}^{*}
\end{aligned}
$$

From the ACE market clearing condition,

$$
H_{x}^{*}=A_{x}
$$

the ACE price for stock $x$ can be obtained,

$$
\begin{aligned}
m_{x}^{*} & =\max \left[1, \beta_{x}\right]\left\{p_{x}-c_{x}-d_{x} \max \left[0,1-\frac{1}{\beta_{x}}\right]\right. \\
& +\alpha\left(p_{y}-c_{y}-m_{y}^{*} \min \left[1, \frac{1}{\beta_{y}^{B}}\right]-d_{y} \max \left[0,1-\frac{1}{\beta_{y}^{B}}\right]\right) \\
& \left.-\frac{w^{B}(1+\alpha)^{2}}{x}\left(\frac{A_{x}}{n^{B}}\right)\right\},
\end{aligned}
$$

where stock $y$ 's ACE price is defined by (C.6). For convenience, it is stated here,

$$
m_{y}^{*}=\max \left[1, \beta_{y}^{T}\right]\left\{p_{y}-c_{y}-d_{y} \max \left[0,1-\frac{1}{\beta_{y}^{T}}\right]-\frac{w^{T}}{y}\left(\frac{A_{y}}{n^{T}}\right)\right\} .
$$

Note that the ACE prices (4.21) and (4.22) are equal to the average (from the homogeneity assumption) marginal profit associated with harvesting an extra unit of fish. This is consistent with the literature, see e.g. Marchal et al. (2011); Little et al. (2009); Guyader \& Thébaud (2001). 
Marginal profit and therefore the ACE price increase with a higher price of fish and lower costs of fishing. Additionally, the following relationships are observed:

- $\frac{\partial m_{x}^{*}}{\partial A_{x}}<0$ and $\frac{\partial m_{y}^{*}}{\partial A_{y}}<0$

This may be explained as follows. A rise in the supply of ACE from increasing the TACC results in the flooding of the market with ACE, which consequently pushes the market price down.

- $\frac{\partial m_{x}^{*}}{\partial x}>0$ and $\frac{\partial m_{y}^{*}}{\partial y}>0$

This may be explained as follows. A rise in stock abundance for a given supply of ACE implies an increase in the amount of harvest for a given level of effort, creating an incentive for all agents to buy more ACE to cover the additional units of catch. This yields an increase in the ACE price.

Now that the ACE price for both fish stocks have been determined, the focus is now on presenting the system's dynamics.

\subsubsection{The system's dynamics}

The system is described by four state variables: target biomass $-x(t)$, bycatch biomass $-y(t)$, target stock's TACC $-A_{x}(t)$, and by-catch stock's TACC $-A_{y}(t)$. The system is modelled over an infinite time horizon,

$$
t \in \Theta \equiv[0, \infty)
$$




\section{The population dynamics}

The population dynamics of stocks $x$ and $y$ are similar to those presented in (3.3) in the previous chapter, in that both stocks are subject to logistic growth in continuous time,

$$
\left\{\begin{array}{l}
\dot{x}(t)=r_{x} x(t)\left(1-\frac{x(t)}{L_{x}}\right)-H_{x}(t) \\
\dot{y}(t)=r_{y} y(t)\left(1-\frac{y(t)}{L_{y}}\right)-H_{y}(t)
\end{array}\right.
$$

where $r_{x}$ and $r_{y}$ denote the intrinsic growth rates, and $L_{x}$ and $L_{x}$ are the environment's carrying capacities, of $x$ and $y$, respectively. Withdrawals of stocks $x$ and $y$ from the system are represented by $H_{x}(t)$ and $H_{y}(t)$, respectively.

Withdrawal of stock $x$ in period $t$ is obtained by summing up (4.1) across all agents in $F^{B}$,

$$
\begin{aligned}
H_{x}(t) & =\sum_{i=1}^{n^{B}} \beta_{x} a_{i x}(t) \\
& =\beta_{x} A_{x}(t) .
\end{aligned}
$$

Note that the sum of individual ACE holdings at the end of the fishing period must add up to the TACC set the beginning of the fishing period.

Withdrawal of stock $y$ in period $t$ is made up of harvests of stock $y$ from both $F^{B}$ and $F^{T}$,

$$
H_{y}(t)=H_{y}^{B}(t)+H_{y}^{T}(t)
$$

In $F^{B}$, total harvest of stock $y$ is obtained by aggregating (4.3) for all agents,

$$
\begin{aligned}
H_{y}^{B} & =\sum_{i=1}^{n^{B}} \alpha \beta_{x} a_{i x}(t) \\
& =\alpha \beta_{x} A_{x}(t) .
\end{aligned}
$$


In $F^{T}$, total harvest of stock $y$ is obtained by aggregating (4.2) for all agents,

$$
\begin{aligned}
H_{y}^{T} & =\sum_{i=1}^{n^{T}} \beta_{y}^{T} a_{j y}(t) \\
& =\beta_{y}^{T} A_{y}(t) .
\end{aligned}
$$

Given (4.27) and (4.28), withdrawal of stock $y$ now reads as follows,

$$
H_{y}(t)=\alpha \beta_{x} A_{x}(t)+\beta_{y}^{T} A_{y}(t)
$$

\section{TACC adjustments}

To fully describe the dynamics of the fisheries model, the population dynamics for stocks $x$ and $y$ (4.24) is coupled with the law of motion for both TACCs, $A_{x}$ and $A_{y}$. It is proposed that the time variability of $A_{x}$ and $A_{y}$ are described by the following differential inclusions,

$$
\left\{\begin{array}{l}
\dot{A}_{x}(t)=u_{x}(t) \in\left[-\delta_{x}, \delta_{x}\right] \equiv U_{x} \\
\dot{A}_{y}(t)=u_{y}(t) \in\left[-\delta_{y}, \delta_{y}\right] \equiv U_{y}
\end{array}\right.
$$

where the parameters $\delta_{x}>0$ and $\delta_{y}>0$ define the maximum speed by which the TACCs can be adjusted between fishing periods. The inclusions represent bounds on the speed at which the regulator can change the TACCs. This reflects the regulator's policy for "smooth" annual catch limit adjustments. 
Thus, the dynamical system for the output-controlled fisheries model is now completed. It is defined through the following vector of differential inclusions,

$$
\left\{\begin{aligned}
\dot{x}(t) & \in r_{x} x(t)\left(1-\frac{x(t)}{L_{x}}\right)-\beta_{x} A_{x}(t) \\
\dot{y}(t) & \in r_{y} y(t)\left(1-\frac{y(t)}{L_{x}}\right)-\alpha \beta_{x} A_{x}(t)-\beta_{y}^{T} A_{y}(t) \\
\dot{A}_{x}(t) & \in U_{x} \\
\dot{A}_{y}(t) & \in U_{y} .
\end{aligned}\right.
$$

\subsubsection{The viability constraints}

Having defined the system's dynamics, the current section defines the viability constraints, which need to be satisfied by the dynamical system at all times. By doing so, the system is maintained in a perennial state. The QMS was introduced into New Zealand's fisheries to achieve two broad goals. Firstly, to limit catches to levels that will result in maximum production from the stock and secondly to maximise the net economic return to the nation (Annala, 1996). Here, adjusted ecological and economic objectives coupled with a social objective are proposed.

\section{The ecological constraint}

Again, the ecological sustainability of the system requires that stocks $x$ and $y$ both do not fall below their respective SMBLs, $x_{\min }>0$ and $y_{\min }>0$,

$$
\left\{\begin{array}{l}
x(t) \geq x_{\min } \\
y(t) \geq y_{\text {min }}
\end{array} \quad \forall t \in \Theta .\right.
$$




\section{The economic constraint}

The economic sustainability of the system requires that the fishing industry remains profitable. Rather than imposing a non-negative condition on profit as in the input-controlled fishery model, here a non-negative condition is imposed on the ACE price for both stocks, which follows from Péreau et al. (2012). Given that the ACE price reflects marginal profit as noted earlier, this condition ensures that the marginal profit of harvesting an additional unit fish is never negative. Moreover, it does not make economic sense to have a negative price of ACE as this would imply subsidising others to buy your ACE.

Thus, the economic constraint is defined as,

$$
\left\{\begin{array}{l}
m_{x}^{*}(t) \geq 0 \\
m_{y}^{*}(t) \geq 0
\end{array} \quad \forall t \in \Theta,\right.
$$

where $m_{x}^{*}(t)$ and $m_{y}^{*}(t)$ are defined in (4.21) and (4.22), respectively.

\section{The social constraint}

Social objectives are often associated with employment and income distribution, production of nutritional food, and maintenance of traditional livelihoods and communities (Hilborn, 2007). The system provides a flow of consumption and services through harvesting, which can either directly or indirectly achieve these objectives. Thus, the social constraint here requires that the annual catch limit (i.e. TACC) on both stocks is never set below a minimum guaranteed harvest level (MGHL),

$$
\left\{\begin{array}{l}
A_{x}(t) \geq H_{x, \text { min }} \\
A_{y}(t) \geq H_{y, \text { min }}
\end{array} \quad \forall t \in \Theta .\right.
$$


Moreover, the rigidity constraint imposed on the speed at which the TACCs can be adjusted (4.30) may reflect some social (and political) cost. As mentioned earlier, under a QMS quota owners have an exclusive right to fish, in perpetuity, a share of the TACC. This property right is also divisible, transferable and bankable, which essentially makes it an asset that promises to generate a flow of economic benefits over time (Sumaila, 2010; Squires et al., 1998). So, any major changes to the TACC may entail significant socio-political ramifications. It is not surprising then that the TAC/TACC for many fish stocks remain constant from year to year despite official recommendations for adjustments (Lock \& Leslie, 2007; Sanchirico et al., 2006).

It is worth mentioning here that associated with any ITQ-based management regime is the issue concerning the concentration of quota by a small group of quota owners (Stewart \& Callagher, 2011), an inevitable consequence of rationalisation. This may pose significant socio-economic costs particular on small-scale fishing operators who have little choice but to leave the fishery because they do not have sufficient quota to cover their catch as mostly all of the quota is controlled by a small group operators ${ }^{15}$. The model developed here cannot deal with this issue given the homogeneity assumption on all agents. Nonetheless, it is worth exploring further in future research.

\footnotetext{
${ }^{15}$ This issue also features in a goema beat by Barry van Zyl, see (van Zyl, 2012).
} 
Thus, using (4.30), (4.32), (4.33) and (4.34), the viability constraint set is defined by,

$$
K \equiv\left\{\begin{array}{l|l}
\left(x, y, A_{x}, A_{y}, u_{x}, u_{y}\right): & \begin{array}{l}
x(t) \geq x_{\min } \\
y(t) \geq y_{\min } \\
m_{x}^{*}(t) \geq 0 \\
m_{y}^{*}(t) \geq 0 \\
A_{x}(t) \geq H_{x, \min } \\
A_{y}(t) \geq H_{y, \min } \\
u_{x}(t) \in U_{x} \\
u_{y}(t) \in U_{y}
\end{array}
\end{array}\right\}
$$

\subsubsection{The viability kernel}

The viability kernel for the output-controlled viability model is defined as follows,

$\mathcal{V} \equiv\left\{\left(x(0), y(0), A_{x}(0), A_{y}(0)\right): \mid \begin{array}{c}\exists\left(u_{x}(\cdot), u_{y}(\cdot)\right) \text { and }\left(x(\cdot), y(\cdot), A_{x}(\cdot), A_{y}(\cdot)\right), \\ \text { starting from }\left(x(0), y(0), A_{x}(0), A_{y}(0)\right) \\ \text { satisfying dynamics }(4.31) \\ \text { and constraints }(4.35) \\ \forall t \in \Theta\end{array}\right\}$,

which delineates the loci of states, from which moderate TACC adjustments can guarantee a balanced evolution of the system. 


\subsection{The model calibration}

\subsubsection{The base parameter set}

The values assigned to the biological parameters (i.e. $r_{x}, r_{y}, L_{x}$ and $L_{y}$ ) and the technical parameter (i.e. $\alpha$ ) here are identical to those used in the input-controlled fishery model, refer to Section 3.3 for the rationale behind these values.

Similarly, the ex-vessel prices of stocks $x$ and $y$ are identical to those used in the preceding chapter. These prices are based on port price of fish stock TAR2, i.e. tarakihi (Nemadactylus macropterus) caught in QMA2, see Table C.1 in the Appendix to this chapter. Port prices are collected on a voluntary basis through annual surveys with licensed fish receivers. These prices are averaged using a 3 year moving average, which is the reason for showing little to no variation between fishing years. Port prices in general are not very reliable because they do not discriminate between fishing methods, market fish sizes, fish quality and season (Lallemand, 2013). They are however the best available indication of ex-vessel prices and are widely used by fisheries managers in New Zealand.

In practice, the deemed value is generally set proportionally to the exvessel price of the fish stock, and it is mostly set higher than the ACE price so as to provide an incentive to agents to cover their catch with ACE, but lower than the ex-vessel price to discourage illegal discarding (Holland \& Herrera, 2006). Newell (2004) shows that the base deemed value is set as low as $10 \%$ of the port price for fish stocks with relatively little information on the fishery status or no sustainability concerns, and as high as $200 \%$ of the port price for some high-valued stocks taken primarily with little, if any, 
by-catch. The deemed values are then, in most cases, modified to account for changes in port prices or if catch levels exceed the TACC.

Here, the (base) deemed values $d_{x}$ and $d_{y}$ are set at $75 \%$ of $p_{x}$ and $p_{y}$, respectively. This is based on the deemed value of TAR2, which remains at about three-quarter of the port price, see Figure C.1 in the Appendix to this chapter. The deemed values are assumed to be fixed over time. This is not an unreasonable assumption given that the deemed values for many fish stocks remain unchanged since the introduction of the DVS in 2001 (Lallemand, 2013).

The values assigned to the unit costs of fishing are not based on actual costs. However, they have been set lower than the ex-vessel prices to ensure that fishing is still profitable and hence worthwhile after taking into account the cost of covering catch with $\mathrm{ACE}$. The unit costs (i.e. $c_{x}$ and $c_{y}$ ) associated with the linear cost component are set at $\$ 1.50 / \mathrm{kg}$ and $\$ 1 / \mathrm{kg}$, respectively. Given that the target stock is more valuable and it is associated with higher catch rates, it is reasonable to assume that it is more costly to harvest a unit of stock $x$ than it is to harvest a unit of stock $y$. For instance, there is more care and time given to sorting and handling target catches. As for the cost parameters $w^{B}$ and $w^{T}$ associated with non-linear variable costs, they are set at $\$ 500$ and $\$ 250$, respectively.

The number of fishing permit holders for the TAR2 fishery for the period 2003-04 to 2009-10 is summarised in Table C.2. Using these figures as a benchmark, the number of agents $n^{B}$ and $n^{T}$ are set at 30 and 20, respectively.

The calibrated values for the output-controlled fisheries model are summarised in Table C.3. These parameter values, in broad terms, characterise a typical multi-species fishery in which the by-catch stock is considerably less 
productive than the target stock, so the former is at risk of over-exploitation if exploited at rates appropriate for the target stock. Thus, it is of particular interest to use viability theory to determine the loci of states, from which the regulator can make the necessary TACC adjustments that guarantee the good health of resource stocks while not neglecting its other economic and social responsibilities.

\subsubsection{The boundaries}

VIKAASA requires that both the lower and upper limits be specified for all state variables $x(t), y(t), A_{x}(t), A_{y}(t)$, and control variables $u_{x}(t)$ and $u_{y}(t)$.

The boundaries for the biological variables $x(t)$ and $y(t)$ proposed here are identical to those used for the input-controlled fishery model. That is, for each stock, the lower limit or the SMBL is set equal the hard limit, i.e. $10 \%$ of the stock's carrying capacity. The upper limit is set equal to the stock's carrying capacity.

The lower bound of the TACC for each stock is defined by the MGHL, which is set arbitrary equal to a $\frac{1}{3}$ of the stock's MSY ${ }^{16}$. Theoretically, levels of catch above MSY cannot be sustained without depleting the resource stock. This makes MSY an appropriate level to set the upper bound for the TACC for each fish stock.

Thus, the proposed boundaries are defined as follows,

- target biomass again is set between $10 \%$ and $100 \%$ of the target stock's carrying capacity, i.e. $x(t) \in[60,600]$;

\footnotetext{
${ }^{16}$ For the logistic growth model, MSY is defined by (A.8), i.e. $Y_{\mathrm{MSY}}=\frac{r L}{4}$, where $r$ and $L$ are the stock's intrinsic growth rate and carrying capacity, respectively.
} 
- by-catch biomass should also be between $10 \%$ and $100 \%$ of the bycatch stock's carrying capacity, i.e. $y(t) \in[30,300]$;

- target TACC should be between $\frac{1}{3}$ and $100 \%$ of the target stock's MSY, i.e. $A_{x}(t) \in[20,60]$;

- by-catch TACC should be between $\frac{1}{3}$ and $100 \%$ of the by-catch stock's MSY, i.e. $A_{y}(t) \in[5,15]$

- target TACC adjustment speed, rather arbitrary the amount by which the regulator can change $A_{x}(t)$ between fishing periods will be between -1 and 1 percentage points, so $u_{x}(t) \in[-0.01,0.01]$; and

- by-catch TACC adjustment speed, similarly the amount by which the regulator can change $A_{y}(t)$ between fishing periods will also be between -1 and 1 percentage points, so $u_{y}(t) \in[-0.01,0.01]$.

\subsection{A viability analysis}

\subsubsection{How to analyse viability kernels with more than three dimensions?}

The fisheries viability problem presented in the current chapter deals with four state variables $\left(x, y, A_{x}\right.$ and $\left.A_{y}\right)$, so $\mathcal{V} \subset K \subset \mathbb{R}^{4}$. The $4 \mathrm{D}$ viability kernel $\mathcal{V}$ is also computed over a range of values of $\beta_{x}$ and $\beta_{y}^{T}$, which are initially kept constant. This allows for a qualitative analysis of the systemwide implications of agents under-catching, matching or over-catching their ACE holdings. The subsequent analysis focuses mostly on the last two cases. The first case, which is referred henceforth as the base scenario considers 
$\beta_{x}=\beta_{y}^{T}=1$. That is, catches by agents in both the by-catch fishery, $F^{B}$ and the target fishery, $F^{T}$ never deviate from their ACE holdings. The second case considers $\beta_{x}=1.2$ and $\beta_{y}^{T}=1$. That is, only agents in $F^{B}$ exceed their ACE by the maximum allowable amount. The analysis also considers a third case where $\beta_{x}$ is allowed to vary between $\beta_{x}(t) \in[0.8,1.2]$, while $\beta_{y}^{T}=1$ remains unchanged. Given that more than three state spaces cannot be visually displayed, the analysis will be conducted using $3 \mathrm{D}$ and sometimes $2 \mathrm{D}$ slices of $\mathcal{V}$, for a given value of $\beta_{x}$ and $\beta_{y}^{T}$. An explanation of how to interpret multi-dimensional kernels is provided in Box 5 .

\section{Box 5. How to interpret $3 D$ slices of the $4 D$ kernel?}

In order to examine the viability configurations for the output-controlled fisheries model, 3D slices of the 4D kernel will be used. The first such slice is shown in Figure 4.2a. The three dimensions, for which the slice is cut, are labelled along the respective axes (here: $x, y$ and $A_{x}$ ); the fourth dimension is kept constant (here: $A_{y}=$ all). The box delimits the three-dimensional projection of $K \subset \mathbb{R}^{4}$, where $K$ is defined by (4.35) as the state constraint set, within which the fishery is supposed to remain, less the economic constraint, which is not displayed. The 3D body ("boulder") is a snapshot of the viability kernel taken for a particular value or values of the fourth dimension, written down in the caption. If there is a line (trajectory) shown in the figure, then each point of this line corresponds to a different value of the fourth dimension; it can therefore be said that the 3D line is parameterised in the fourth dimension.

From the viability kernel's definition (2.7), it is known that: 
- for each initial state represented as a point in the boulder, there exists smooth TACC policies $\left(u_{x} \in U_{x}\right.$ and $\left.u_{y} \in U_{y}\right)$, which maintain the fishery in the viability constraint set $K$;

- the points outside the boulder are those states that cannot be controlled to remain in $K$ by these policies.

\subsubsection{Population viability analysis for the base sce- nario}

As a starting point, selected slices of the viability kernel computed without the economic constraint (4.33) also known as the "population viability kernel" are presented in Figure 4.2. There are four possible 3D slices of the 4D kernel, however only two are shown here. The shaded 3D boulders represent available choices of the TACCs, $A_{x}$ (left panel) and $A_{y}$ (right panel) that satisfy both the ecological and social constraints (4.32) and (4.34), for the base scenario $^{17}$.

There are two important observations that are noted for Figure 4.2. Firstly, the SMBL chosen for stock $y$ is non-viable for any given value of the other three state variables. In other words, the ecological sustainability of the system requires that the size of stock $y$ 's biomass be maintained at levels that are higher than the hard limit. This is so stock $y$ is sufficiently

\footnotetext{
${ }^{17}$ Note that under the base scenario, agents in both fisheries always catch their ACE holdings (i.e. $\beta_{x}=\beta_{y}^{T}=1$ ). Consequently, total harvest of the target stock $x$ is always equal to the stock's TACC, $A_{x}$. However, the TACC, $A_{y}$ for the by-catch stock is always over-caught by the amount of stock $y$ harvested in the by-catch fishery. So deemed value payments are only limited to the by-catch harvest.
} 


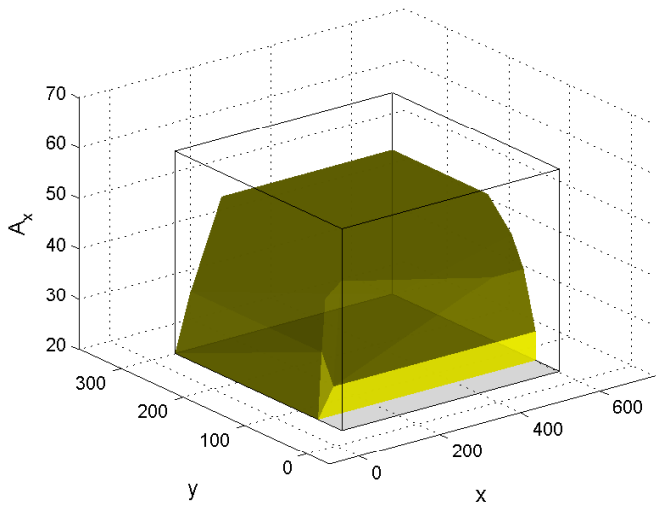

(a) Viable choices of $A_{x}$

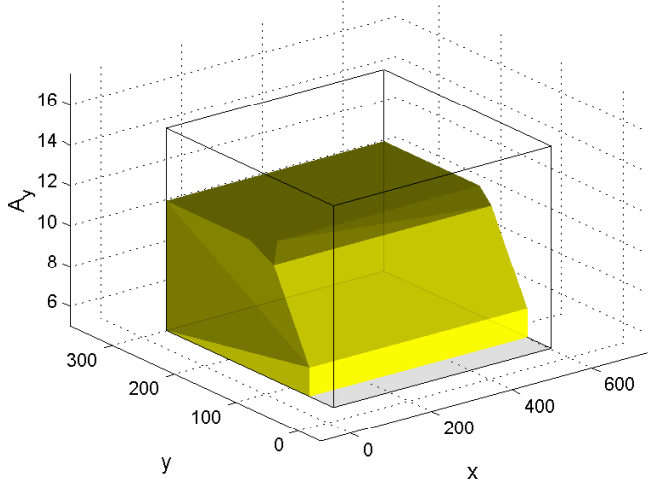

(b) Viable choices of $A_{y}$

Figure 4.2: The population viability kernel for the base scenario. Panel (a) presents a $3 \mathrm{D}$ slice of the kernel through all values of $A_{y}$. Panel (b) presents a $3 \mathrm{D}$ slice of the kernel through all values of $A_{x}$.

productive to cope with the additional pressure imposed by the incidental catch of $y$ by the fishing fleet in $F^{B}$. Secondly, setting the TACCs for stocks $x$ and $y$ at their respective MSY levels (i.e. upper bounds) is not a viable policy. In fact, the choices of $A_{x}$ and $A_{y}$ are constrained to values lower than their upper bounds. Similarly to the explanation for the first observation, this is so that total withdrawals of stock $y$ from both fisheries are maintained at levels that do not put this stock's population at risk of extinction.

Setting the TACC equal to MSY as a non-viable policy is an interesting result particularly from the regulator's perspective given that MSY is a common management target (Hilborn, 2007). It is common practice in multi-species fisheries to set catch limits independently, with little or no consideration of co-dynamics of fish stocks (Holland \& Herrera, 2006). Here, the implications of managing fish stocks independently are highlighted. The 
two-species population viability kernel (i.e. Figure 4.2) projected onto the $A_{x}$ vs $x$ planes is contrasted with that generated for the single-species case. The relevant viability domains are presented in Figure 4.3.

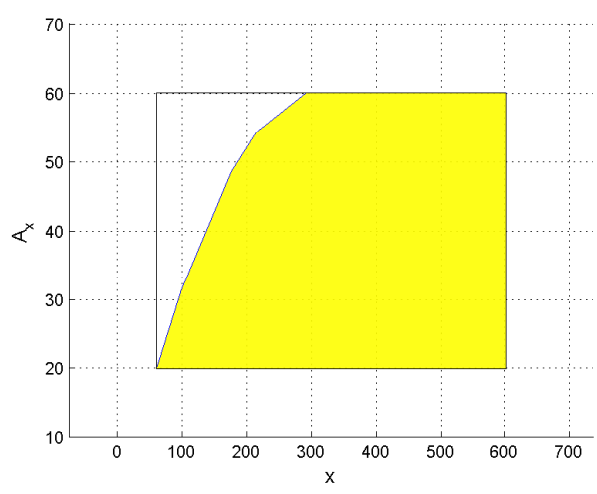

(a) Single-species case

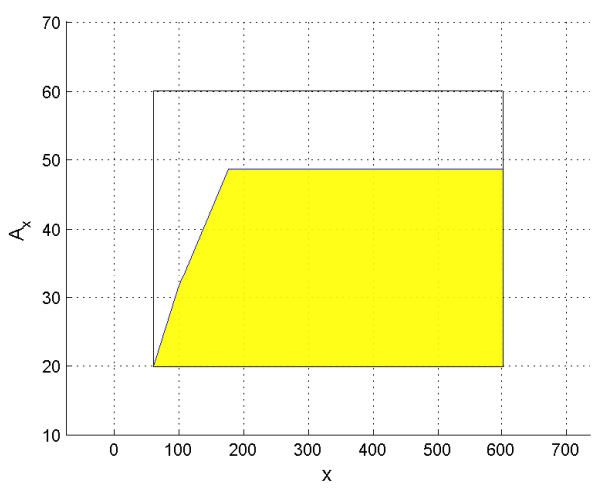

(b) Two-species case

Figure 4.3: A contrast of the choice of viable TACC policies for stock $x$ under the single-species and two-species species specification, for the base scenario. Panel (a) considers only the ecological sustainability of stock $x$, and panel (b) considers the ecological sustainability of stocks $x$ and $y$ conjointly.

Under the single-species specification, MSY is confirmed as a viable TACC policy provided that the size of $x$ is maintained at a level that is at least a half of the stock's carrying capacity ${ }^{18}$, see Figure $4.3 \mathrm{a}$. Given this result, it is not surprising that setting catch limits equal to MSY is a common management target. However, when taking into consideration the impact of joint production on the ecological sustainability of the slower growing stock $y$, the choice of viable TACC policies becomes constrained to levels lower than MSY

\footnotetext{
${ }^{18}$ For the logistic growth model, the stock's biomass that supports MSY is theorectically equal to $x_{\mathrm{MSY}}=\frac{L_{x}}{2}$, see (A.9).
} 
as shown in Figure 4.3b.

For a closer look at the viable choices of TACCs available to the regulator, the population viability kernel is projected onto the $A_{x}$ vs $A_{y}$ planes for all values of $x$ and $y$, in Figure 4.4. The two TACCs are clearly shown to be constrained well below their respective upper bounds as noted earlier. All combinations of $A_{x}$ and $A_{y}$ that are not in the shaded areas are nonviable because they pose a potential risk to the ecological sustainability of the system.

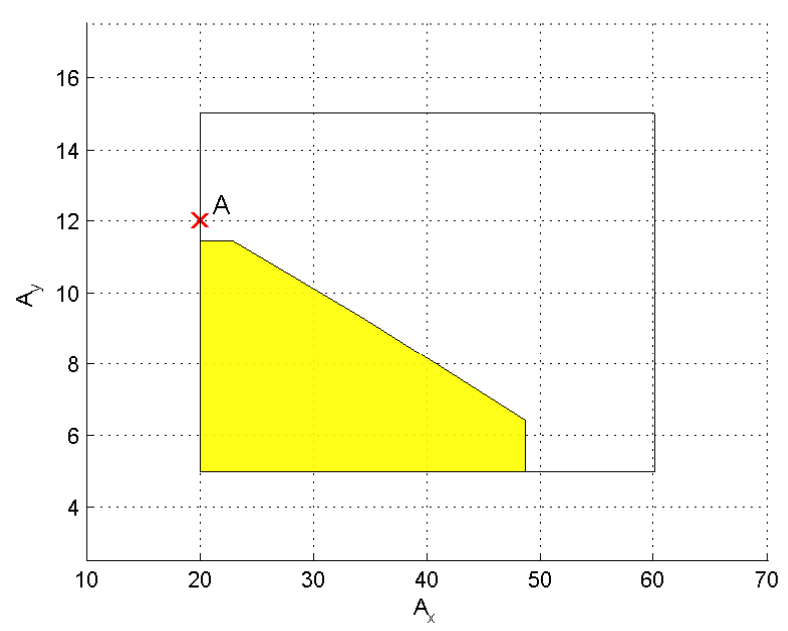

Figure 4.4: A 2D projection of the population viability kernel onto the $A_{y}$ vs $A_{x}$ planes for all values of $x$ and $y$, for the base scenario.

For example, consider the initial state $\left[x(0), y(0), A_{x}(0), A_{y}(0)\right]=$ $[300,150,20,12] \notin \mathcal{V}$ labelled "A" in Figure 4.4 and whose time profiles are presented in Figure 4.5. Starting here inevitably results in stock $y$ 's biomass being driven below its SMBL (see the panel titled " $y$ "), even with $A_{y}$ reduced by the maximum amount allowed (see the panel titled " $u_{y}$ "). This is not unexpected given that total withdrawal of $y$, i.e. $H_{y}(t)=\alpha \beta_{x} A_{x}(t)+$ 
$\beta_{y}^{T} A_{y}(t)$, never falls below MSY, see the dashed blue line, which always remains above $y$ 's upper bound in the panel titled " $A_{y}$ " 19 . Note that $A_{x}$ cannot be reduced any further in order to decrease the by-catch harvest component (i.e. $\left.\beta_{x} A_{x}(t)\right)$ as $A_{x}$ is already equal to its lower bound, and therefore it would violate the social constraint (4.34). It can be verified that any of the states to the right of the shaded area in Figure 4.4 would violate the ecological constraint (4.32).
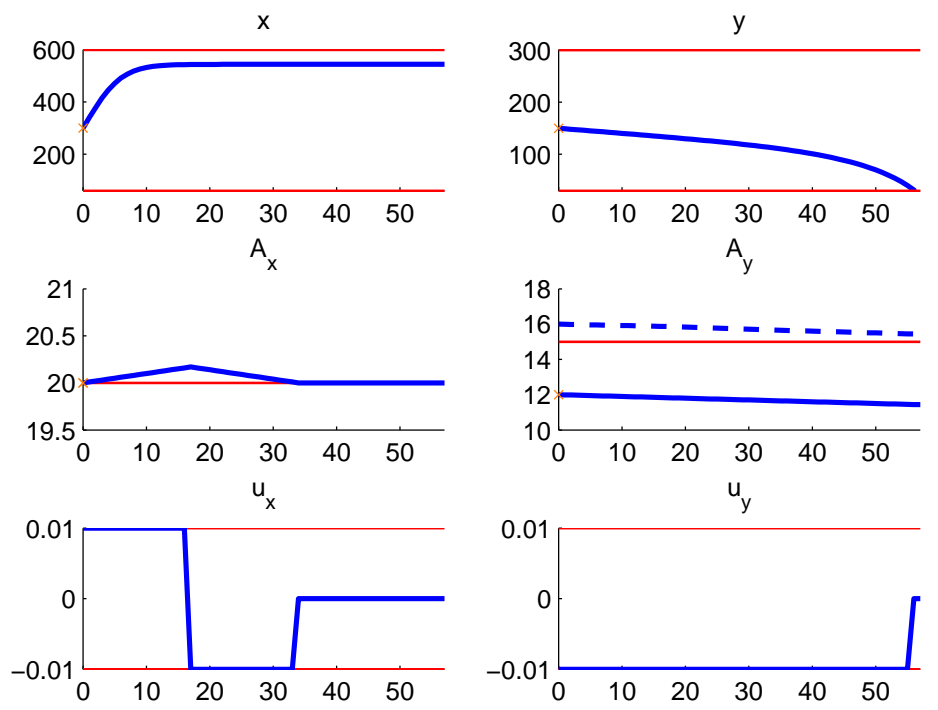

Figure 4.5: Time profiles associated with the initial state "A" marked in Figure 4.4. The red horizontal lines represent the bounds on dynamic variables.

\footnotetext{
${ }^{19} \mathrm{MSY}$ is the biological maximum so any harvest above this level is not ecologically sustainable.
} 


\subsubsection{Co-viability analysis for the base scenario}

The focus now is on providing a qualitative analysis of the effect of incorporating the economic constraint (4.33) coupled with the ecological and social constraints on the viability configurations for the output-controlled fishery problem. The kernel computed with all viability constraints conjointly is called the "co-viability kernel", and it is presented in Figure 4.6. Panels on the left, i.e. Figures $4.6 \mathrm{a}$ and $4.6 \mathrm{c}$ display selected 3D slices of the coviability kernel for the base scenario. For a clearer visualisation of the effect of the economic constraint on the viability space, corresponding 3D slices of the population viability kernel (i.e. without the economic constraint) are presented in the right-hand-side panels, i.e. Figures $4.6 \mathrm{~b}$ and $4.6 \mathrm{~d}$.

The size of the kernel with the economic constraint is relatively smaller than that without the economic constraint, which confirms that the economic condition is constraining ${ }^{20}$. More specifically, the non-negative condition imposed on the ACE prices for the calibrated model constrains $x$ to relatively higher levels, and $A_{x}$ to relatively lower levels. The latter remark is consistent with that made in Péreau et al. (2012), which is done so analytically. These observations imply that the economic constraint entails more conservative restrictions than what would otherwise be ecologically acceptable.

A remark was made earlier that the $\mathrm{ACE}$ price reflected the average marginal profit of extracting an additional unit of fish. It is therefore not surprising to observe that larger stock sizes entail positive ACE prices given that the marginal cost (i.e. non-linear cost component) of harvesting an extra fish unit decreases with increased stock abundance, ceteris paribus, refer to

\footnotetext{
${ }^{20}$ Initial states that are contained in the population viability kernel, but not in the co-viability kernel are associated with negative ACE prices.
} 


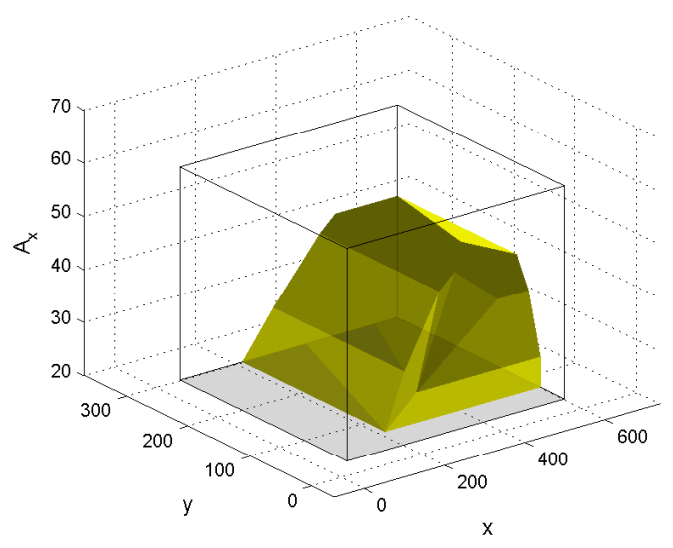

(a) Viable choices of $A_{x}$ with the economic constraint

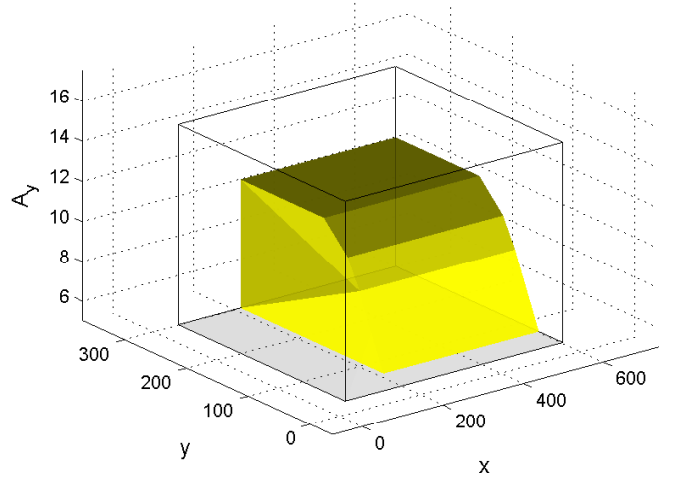

(c) Viable choices of $A_{y}$ with the economic constraint

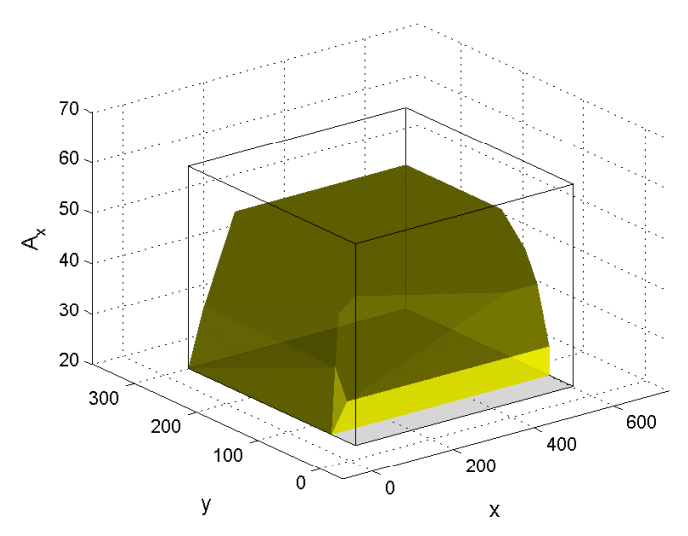

(b) Viable choices of $A_{x}$ without the economic constraint

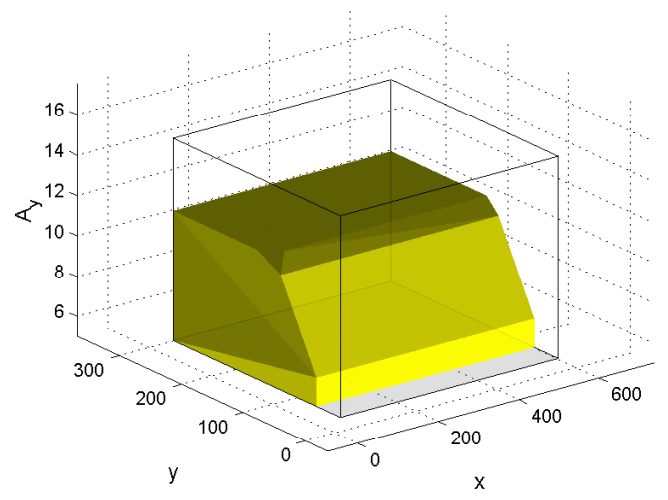

(d) Viable choices of $A_{y}$ without the economic constraint

Figure 4.6: A contrast of selected 3D slices of the co-viability kernel (left panels) with corresponding slices of the population viability kernel (right panels), for the base scenario. 
(4.12) and (4.13). Conversely, higher TACC levels entail higher marginal cost (i.e. also through the non-linear cost component) of harvesting an extra fish unit, ceteris paribus, so positive ACE prices are associated with lower TACC values.

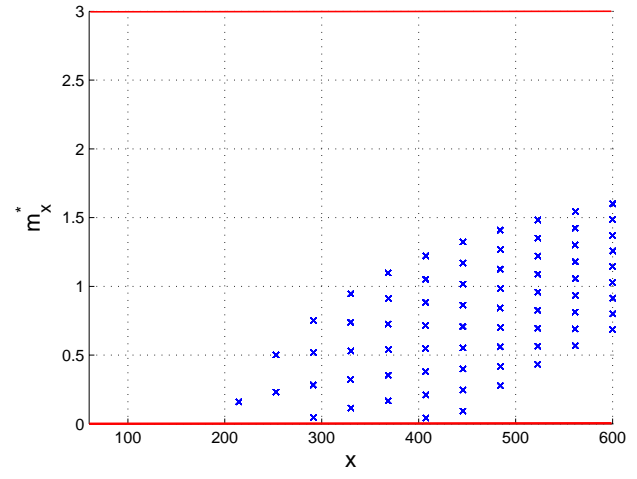

(a) Scatter plot of $m_{x}^{*}$ and $x$

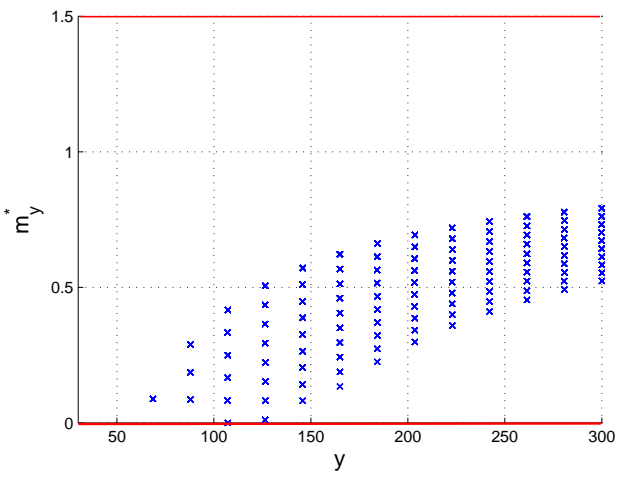

(c) Scatter plot of $m_{y}^{*}$ and $y$

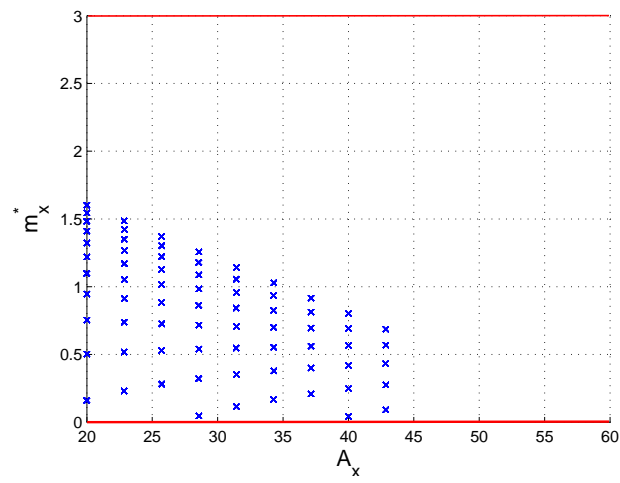

(b) Scatter plot of $m_{x}^{*}$ and $A_{x}$

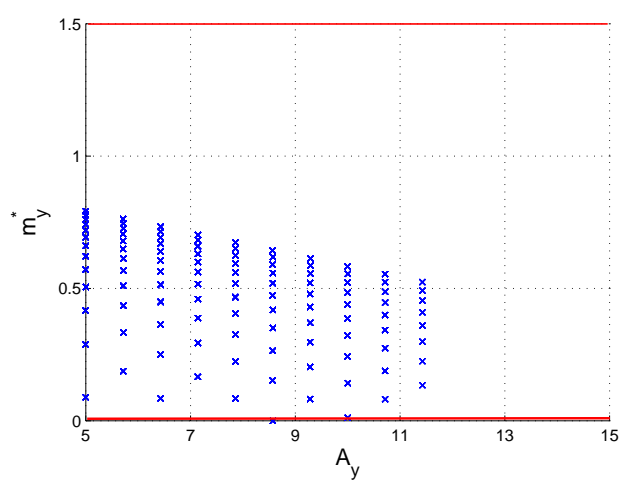

(d) Scatter plot of $m_{y}^{*}$ and $A_{y}$

Figure 4.7: Scatter plots of the ACE prices against selected explanatory variables. The red horizontal lines represent the bounds on the ACE prices, i.e. $m_{x}^{*} \in\left(0, d_{x}\right)$ and $m_{y}^{*} \in\left(0, d_{y}\right)$. 
Figure 4.7 confirms that the ACE prices $m_{x}^{*}$ and $m_{y}^{*}$ corresponding to the co-viability kernel satisfy the economic constraint. Specifically, $m_{x}^{*}$ is positive over the range of viable values of $x$ (approx. $200<x \leq 600$ ) and $A_{x}$ (approx. $20 \leq A_{x}<45$ ), see panels (a) and (b), respectively ${ }^{21}$. Similarly, $m_{y}^{*}$ is also positive over the range of viable values of $y$ (approx. $50<y \leq 300$ ) and $A_{y}$ (approx. $5 \leq y<12$ ), see panels (c) and (d), respectively. The scatter plots also show that $m_{x}^{*}$ is increasing (decreasing) with higher stock $x$ (higher $A_{x}$ ), and that $m_{y}^{*}$ is increasing (decreasing) with higher stock $y$ (higher $A_{y}$ ). These observations confirm the remarks made earlier that $\frac{\partial m_{x}^{*}}{\partial x}>0, \frac{\partial m_{y}^{*}}{\partial y}>0$, $\frac{\partial m_{x}^{*}}{\partial A_{x}}<0$ and $\frac{\partial m_{y}^{*}}{\partial A_{y}}<0$.

\subsubsection{Implications of over-catch on the co-viability ker- nel}

So far, the analysis has focused on the viability kernels generated for the base scenario. The focus here is to analyse the co-viability kernel generated for the case where agents in the by-catch fishery $F^{B}$ over-catch their ACE for the target fish stock by $20 \%$, and consequently the TACC for stock $x$ is also over-caught by the maximum allowable amount of $20 \%$, i.e. $\beta_{x}=1.2$. Given that $A_{y}$ is already over-caught by the amount of stock $y$ caught as by-catch in $F^{B}$, specifying $\beta_{y}^{T}>1$ would not add much value to the analysis, so $\beta_{y}^{T}=1$ remains.

Selected 3D slices of the co-viability kernel generated for $\beta_{x}=1.2$ and $\beta_{y}^{T}=1$ are presented in Figures $4.8 \mathrm{a}$ and $4.8 \mathrm{c}$. For a clearer visualisation of

\footnotetext{
${ }^{21}$ Although it is not shown here, it can also be verified that $m_{x}^{*}$ is positive for viable values of $y$. Note that the latter affects the former rather indirectly via $m_{y}^{*}$, so it is not expected that there is an obvious relationship between $m_{x}^{*}$ and $y$.
} 
the effect of specifying a higher $\beta_{x}$, corresponding 3D slices of the co-viability kernel under the base scenario are presented in Figures $4.8 \mathrm{~b}$ and $4.8 \mathrm{~d}$.

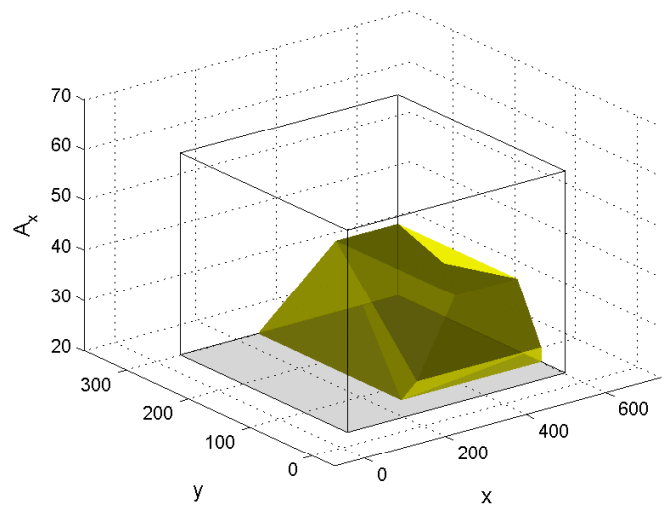

(a) Slice through $A_{y}=$ all, $\beta_{x}=1.2, \beta_{y}^{T}=1$

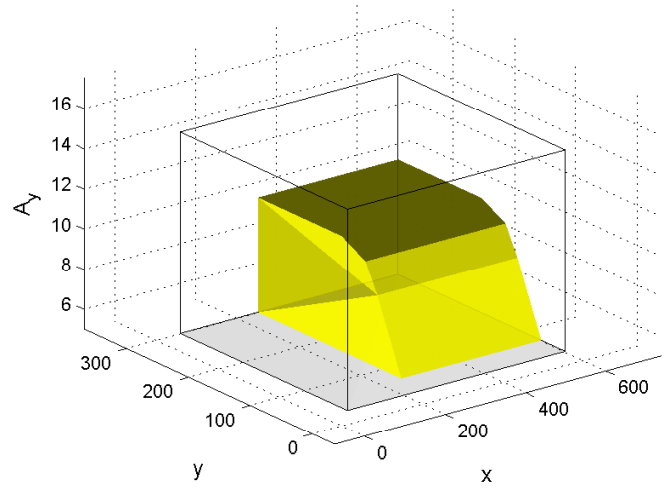

(c) Slice through $A_{x}=$ all, $\beta_{x}=1.2, \beta_{y}^{T}=1$

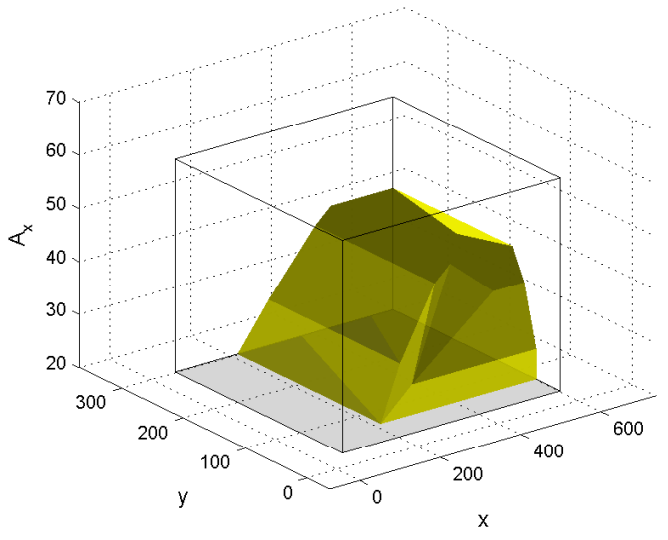

(b) Slice through $A_{y}=$ all, $\beta_{x}=1, \beta_{y}^{T}=1$

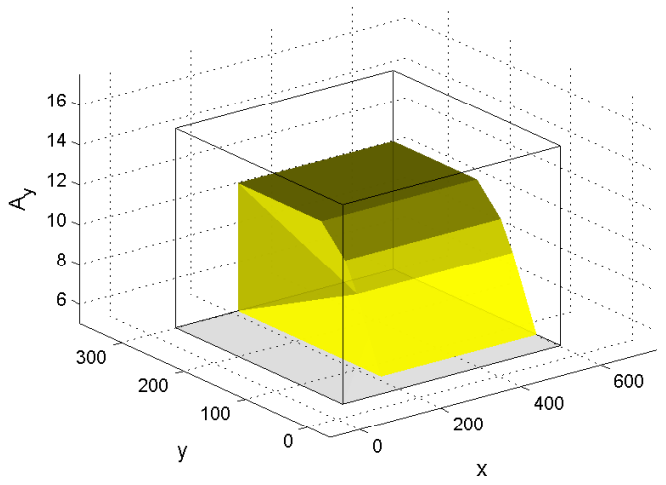

(d) Slice through $A_{x}=$ all, $\beta_{x}=1, \beta_{y}^{T}=1$

Figure 4.8: Selected 3D slices of the co-viability kernel associated with overcatching $A_{x}$ (left panels) and corresponding slices of the co-viability kernel under the base scenario (right panels). 
Firstly, the regulator's choice of $A_{x}$ is much more restricted when the TACCs are over-caught as shown in Figure 4.8a. This is mainly because over-catching $A_{x}$ implies higher by-catch harvest by the fishing fleet in $F^{B}$, which puts additional pressure on the survival of the slower growing by-catch fish stock, $y$. Constraining $A_{x}$ to lower levels is therefore required to ensure the ecological sustainability of stock $y$. Similarly, the choice of values for $A_{y}$ is also shown to be constrained to lower levels, but only slightly as depicted in Figure 4.8c, for the same reason explained above.

Secondly, the biomass of stock $x$ is constrained to much higher levels when its TACC is over-caught. Given that agents in $F^{B}$ are now paying deemed value for catch of the target stock $x$ in excess of their ACE holdings, higher abundance of stock $x$ is required to reduce harvest costs in order to offset the additional costs associated with the deemed value payments, and therefore maintaining the profitability of the fishery.

To further illustrate the economic implications of over-catching $A_{x}$, Figure 4.9 contrasts viable fishing profit levels associated with $\beta_{x}=1.2$ (left panel) with those under the base scenario, $\beta_{x}=1$ (right panel). The profit domain demonstrated here indicates the potential profits for an agent in $F^{B}$ (horizontal axis) and an agent in $F^{T}$ (vertical axis). Profit levels for an agent in $F^{B}$ are much lower when $A_{x}$ is over-caught (i.e. $\beta_{x}=1.2$ ) compared to when it is just-caught (i.e. $\left.\beta_{x}=1\right)^{22}$. This implies that the deemed value specification (i.e. $d_{x}=0.75 p_{x}$ ) for the calibrated model is constraining. It is also noted that over-catching the target stock in $F^{B}$ can potentially constrain profits in $F^{T}$ to lower levels. This is mainly a consequence of restricting $A_{y}$

\footnotetext{
${ }^{22}$ Similarly, it can be easily verified that profits for an agent in $F^{T}$ are constrained to lower levels when $\beta_{y}^{T}=1.2$ is assumed.
} 
to lower levels noted in Figure 4.8c in order to dampen the incidental effect of increased by-catch harvest (i.e. through over-catch of the target stock $x$ ) on the survival of the by-catch stock. This is an example of a "dynamic externality" resulting from the activity of one group of agents, which inflicts costs on another group of agents ${ }^{23}$. Here, such costs include reduced yields and revenues.

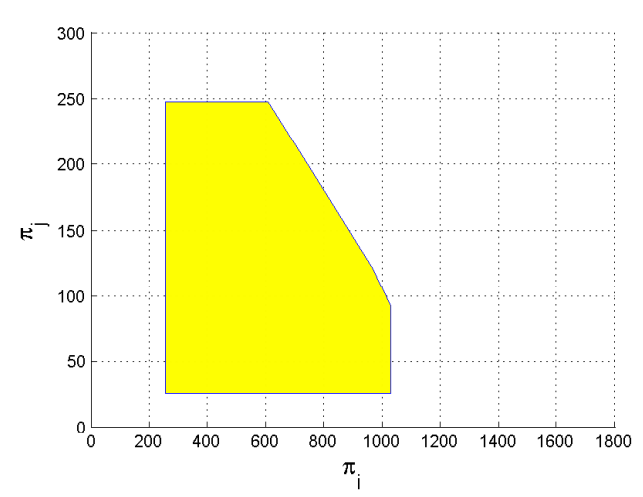

(a) $\beta_{x}=1.2$

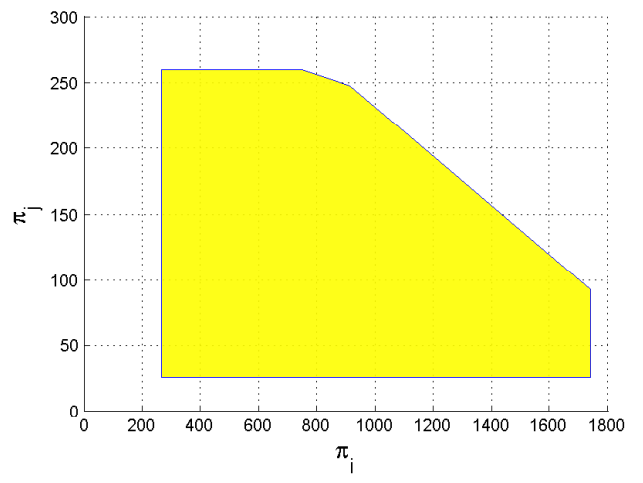

(b) $\beta_{x}=1$

Figure 4.9: Viable fishing profit levels associated with over-catching $A_{x}$ (panel a) and the base scenario (panel b). The horizontal axis reads the profit for agent $i$. The vertical axis reads the profit for agent $j$.

The above shows that when the deemed value is set sufficiently high, agents are better off economically to limit their catch within self ACE holdings, and therefore constraining catches within TACCs. By contrast, when the deemed value is set too low, i.e. lower than the ACE price as shown in Figure 4.10, there are initial states where it is profitable for an agent to overfish

\footnotetext{
${ }^{23}$ Wachsman (2003) categorises the different types of externalises associated with commercially exploited multi-species fisheries into five groups: dynamic, market, biological, spillover and production.
} 


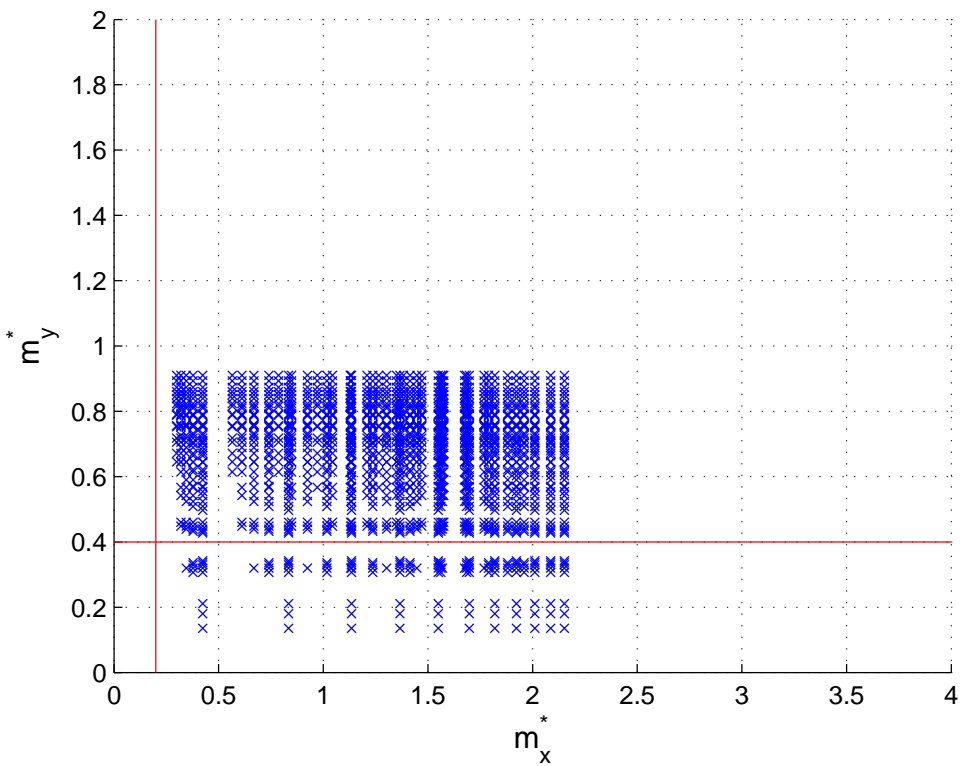

Figure 4.10: Scatter plots of the ACE prices, $m_{x}^{*}$ and $m_{y}^{*}$ for the case where the deemed value is set at $10 \%$ of the port price for both fish stocks. The deemed values $d_{x}$ and $d_{y}$ are represented by the vertical and horizontal red lines, respectively. 
his ACE and cover the excess catch by paying the deemed value, see Figure 4.11. There are examples of agents exploiting New Zealand's catch-balancing system by over-catching a number of fish stocks whose deemed value were set at low levels (Mace et al., 2013). As a consequence, accumulated catches of these stocks were well above their TACC. This has resulted in a concerted effort to set deemed values at appropriate levels in order to discourage agents from intentionally targeting stocks without ACE, while encouraging them to land rather than discard any uncovered catch. In effect, landings have been constrained within the TACC and deemed value payments have dramatically decreased (Mace et al., 2013).

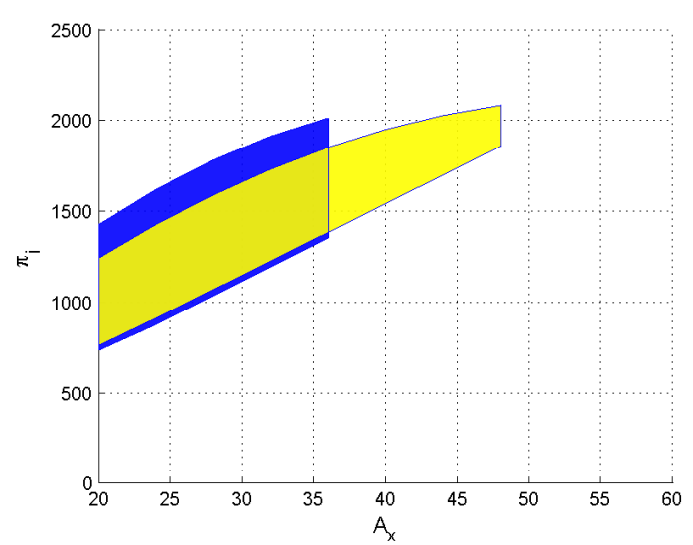

(a) Agent $i$

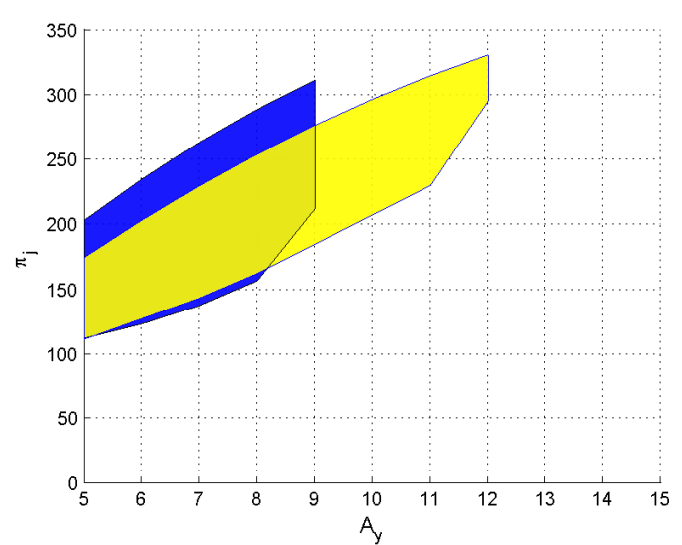

(b) Agent $j$

Figure 4.11: Viable fishing profit levels associated with the deemed value being set at $10 \%$ of the port price for different TACC levels. Panel (a) contrasts agent $i$ 's potential profits obtained for $\beta_{x}=1.2$ (blue area) with those obtained for $\beta_{x}=1$ (yellow area). Similarly, a contrast of agent $j$ 's profits for $\beta_{y}^{T}=1.2$ (blue area) and $\beta_{y}^{T}=1$ (yellow area) is shown in panel (b). 


\subsubsection{Allowing for $\beta_{x}$ to vary over time}

So far the analysis assumes that agents' propensity to deviate from their ACE holding remains constant over time. In reality, the propensity to deviate has some discretionally element and therefore varies over time depending on a number of economic factors, e.g. the cost of covering catch with ACE relative to the deemed value. An agent may also engage in different (or a mixture of different) strategic behaviours, such as those (i.e. "contracted", "independent" and "vertically integrated") characterised in Lallemand (2013). Different strategic behaviours should affect an agent's propensity to acquire ACE and/or deemed value to cover his catch differently. For instance, both vertically integrated and contracted fishing operators follow strict fishing plans so they are less likely to catch in excess of ACE provided in-house or by the contractor ${ }^{24}$. By contrast, independent operators are more likely to fish without ACE (or fish with deemed value) given that they rely on the open market to acquire ACE and therefore they are vulnerable to ACE availability. The transaction cost associated with covering catch with ACE can be prohibitive when ACE availability is restricted either by the TACC being set unnecessarily low or simply by leaving it too late in the fishing season to find ACE.

The strategic behaviour of agents can be modelled by specifying a differential inclusion for $\beta_{x}$ and $\beta_{y}^{T}$ in the output-controlled fisheries model. Note

\footnotetext{
${ }^{24}$ However, if the economic or biological environment permits, e.g. the market price of the targeted fish stock(s) (fuel) is at an all time high (low) or there is a high abundance of fish, it may be worthwhile then for an agent to catch beyond the contracted fishing plan and then search for $\mathrm{ACE}$ on the open market or pay deemed value to cover the additional catch.
} 
that this problem will be in six dimensions, which will require significant computational time to compute the $6 \mathrm{D}$ viability kernel. For convenience, it is assumed here that only agents in the by-catch fishery can vary their behaviour, i.e. their propensity to deviate from their ACE holding can vary over time $\left(\beta_{x}(t)\right)$. As for agents in the target fishery, the assumption that $\beta_{y}^{T}=1$ remains unchanged.

Suppose that agents in the by-catch fishery can under-catch or over-catch their ACE holding by a maximum of $20 \%$ and that the speed of variation of $\beta_{x}(t)$ between fishing periods is between -10 and 10 percentage points, which is sufficiently rigid due to e.g. contractual obligations or limited ACE availability. Thus, the following additions are made to the output-controlled fisheries model,

$$
\begin{aligned}
& \dot{\beta}_{x}(t)=v_{x}(t) \in[-0.1,0.1] \\
& \beta_{x}(t) \in[0.8,1.2] .
\end{aligned}
$$

The viability kernel for the above problem, which is in 5D shall be called the "augmented" co-viability kernel. This is to distinguish it from the coviability kernel computed for the case where agents cannot vary $\beta_{x}$. The focus here is merely to present selected slices of the this 5D kernel and to describe key differences between the "augmented" co-viability kernel and the co-viability kernel in order to highlight the effect of changes in strategic behaviour on the viability of the system.

Figure 4.12 displays selected 3D slices of the augmented co-viability kernel for two cases. Panels on the left, i.e. Figures 4.12a and 4.12c, consider the case where agents in the by-catch fishery initially over-catch their ACE holdings (and consequently the TACC) for stock $x$ by the maximum allow- 
able amount of $20 \%$ (i.e. $\beta_{x}(0)=1.2$ ). By contrast, panels on the right, i.e. Figures $4.12 \mathrm{~b}$ and $4.12 \mathrm{~d}$, consider the base case where agents' ACE holdings (and consequently the TACC) for stock $x$ are initially just-caught (i.e. $\left.\beta_{x}(0)=1\right)$. The selected slices of the augmented kernel for these two cases are not too dissimilar. The notable difference however is that stock $x$ 's biomass is constrained to higher levels when agents in $F^{B}$ initially over-catch their ACE holdings. Higher stock abundance is required to reduce harvest costs in order to offset the additional costs associated with the deemed value payments, and therefore maintaining a positive marginal profit ${ }^{25}$. A comparison of Figures $4.12 \mathrm{a}$ and $4.12 \mathrm{~b}$ shows that there is some (but trivial) restriction on the viable choices of $A_{x}$ available to the regulator. Note that there were significant differences between viable choices of $A_{x}$ for these two cases (i.e. $\beta_{x}=1.2$ and $\beta_{x}=1$ ) when $\beta_{x}$ was assumed constant, refer back to Figures $4.8 \mathrm{a}$ and $4.8 \mathrm{~b}$.

Figure 4.13 provides a comparison of viable states when $\beta_{x}$ can vary (left panels) and when $\beta_{x}$ cannot vary (right panels) for the case where agents (initially) over-catch their ACE holdings for stock $x$. Firstly, there is no obvious change to the range of viable sizes of stocks $x$ and $y$ when agents in $F^{B}$ can vary their strategic behaviour. And secondly, there are notably more choices of higher TACCs (i.e. $A_{x}$ and $A_{y}$ ) for both stocks that are viable when agents can vary their behaviour. That is, it is viable for the regulator to set both $A_{x}$ and $A_{y}$ at higher levels even if agents initially over-catch $A_{x}$. This is because the (sufficiently) high deemed value will induce agents to alter

\footnotetext{
${ }^{25}$ Recall, that agents are liable to deemed value payments when they over-catch their ACE, i.e. $\beta_{x}>1$.
} 


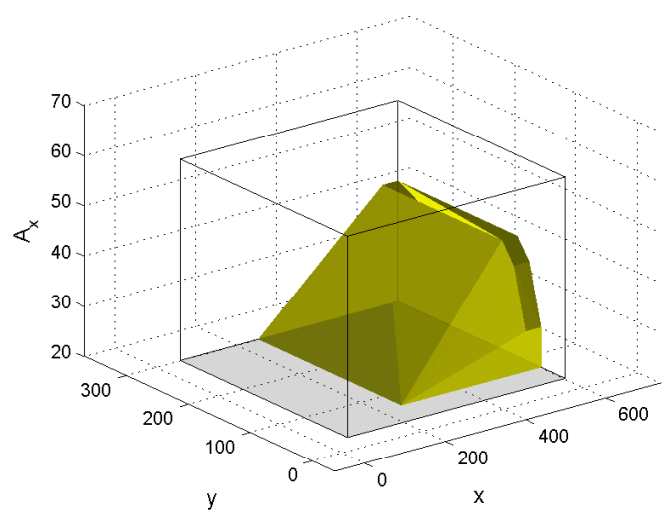

(a) Slice through $A_{y}=$ all and $\beta_{x}(0)=1.2$

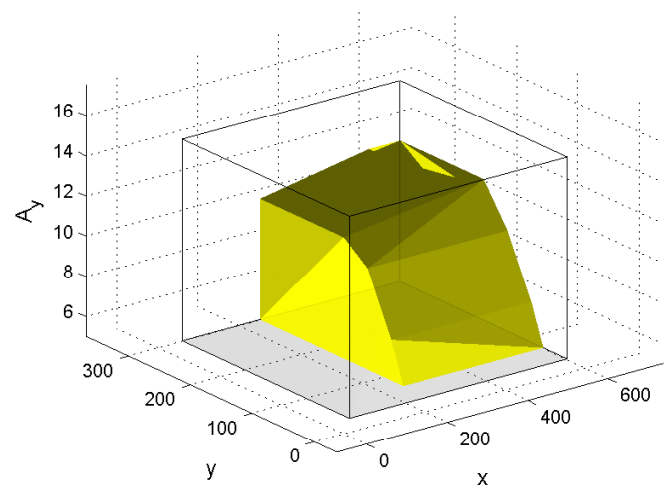

(c) Slice through $A_{x}=$ all and $\beta_{x}(0)=1.2$

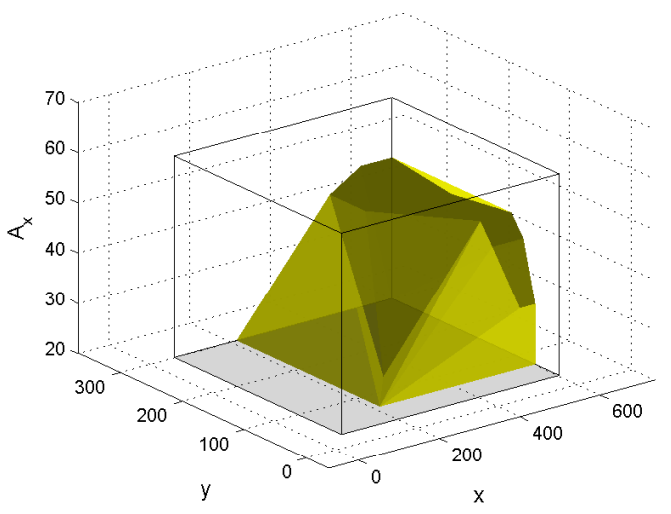

(b) Slice through $A_{y}=$ all and $\beta_{x}(0)=1$

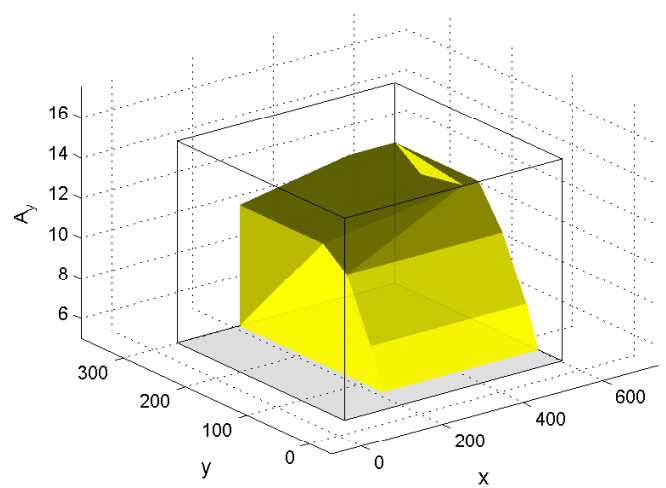

(d) Slice through $A_{x}=$ all and $\beta_{x}(0)=1$

Figure 4.12: Selected 3D slices of the augmented co-viability kernel associated with $\beta_{x}(0)=1.2$ (left panels) and $\beta_{x}(0)=1$ (right panels). 


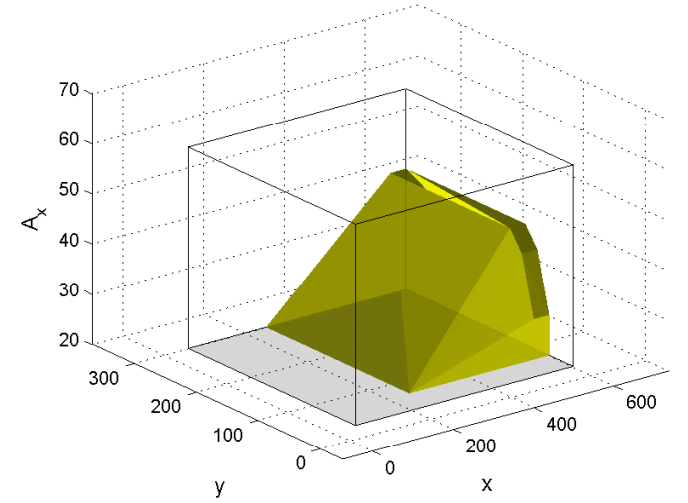

(a) Viable choices of $A_{x}$ when $\beta_{x}$ can vary

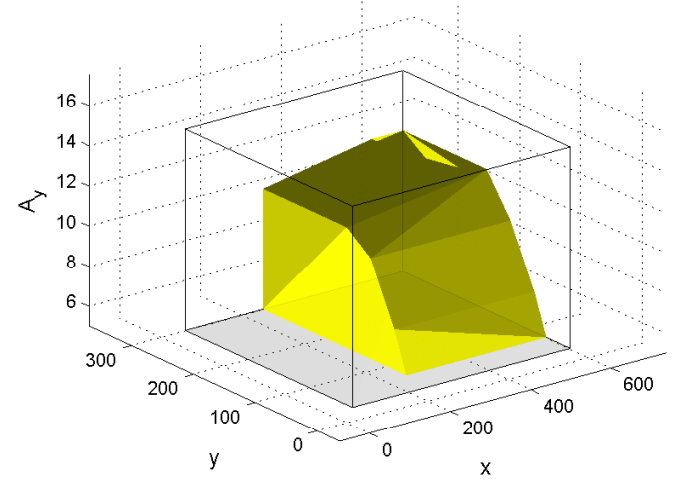

(c) Viable choices of $A_{y}$ when $\beta_{x}$ can vary

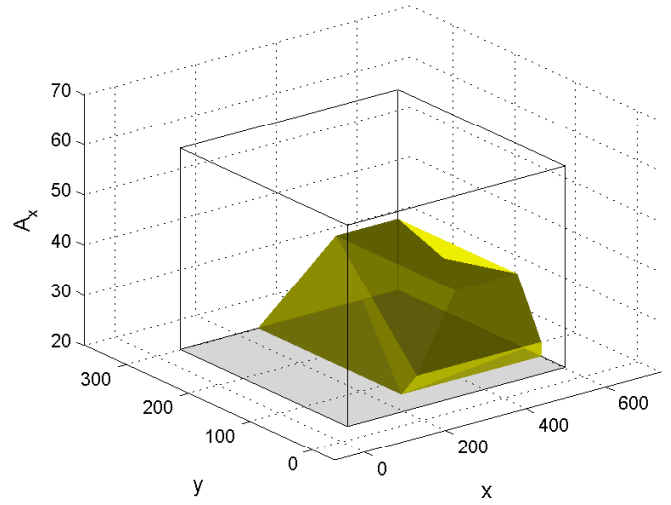

(b) Viable choices of $A_{x}$ when $\beta_{x}$ remains fixed

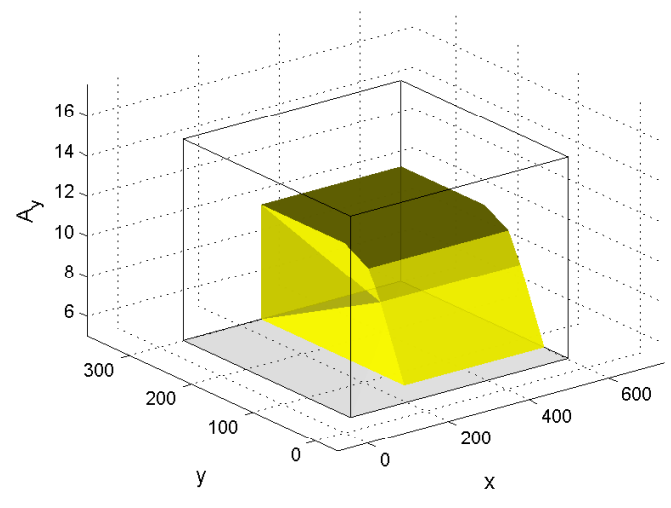

(d) Viable choices of $A_{y}$ when $\beta_{x}$ remains fixed

Figure 4.13: Selected 3D slices of the "augmented" co-viability kernel (left panels) and the co-viability kernel (right panels) when initially beginning with $\beta_{x}=1.2$. 
their behaviour, i.e. reduce $\beta_{x}$ over time, resulting in a flow of sustainable catch rates that do not put the ecological sustainability of the system at risk.

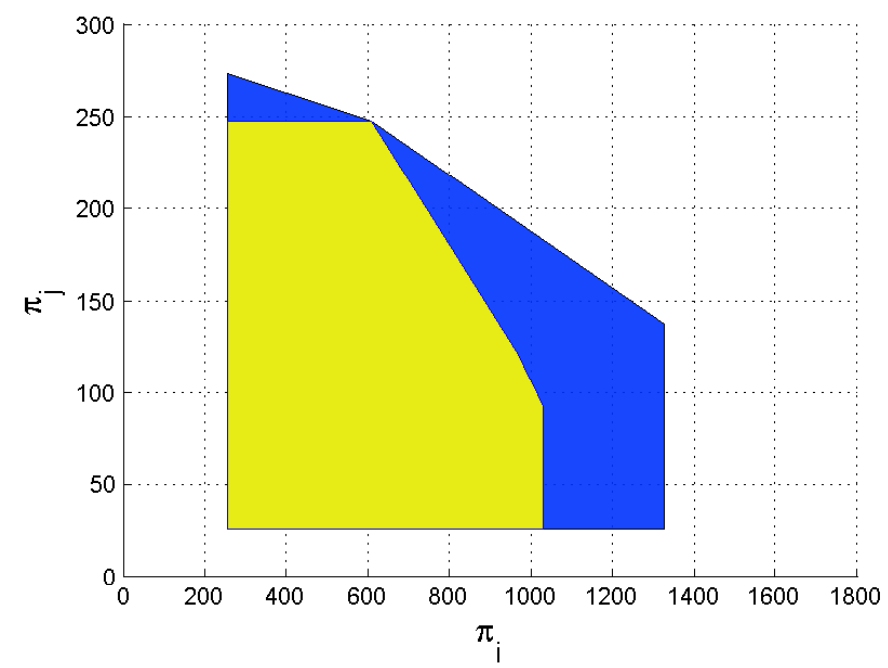

Figure 4.14: The yellow area represents the viable fishing profit domain when initially beginning with $\beta_{x}=1.2$, which remains unchanged at all times. The blue area represents the additional viable profit levels when beginning with $\beta_{x}=1.2$, but which can vary over time. The horizontal axis reads the profit for an agent in $F^{B}$. The vertical axis reads the profit for agent in $F^{T}$.

Moreover, higher viable TACCs imply larger yields and consequently higher profits for all agents in both fisheries as noted in Figure 4.14. Alternatively, in the case where agents cannot alter their behaviour, by starting with $\beta_{x}=1.2$ (i.e. over-catching self $\mathrm{ACE}$ ), agents will always be subject to deemed value payments, which will substantially reduce their cash flow over time. By contrast, when agents are able to alter their behaviour, they can improve their cash flow by reducing $\beta_{x}$ and therefore any deemed value liabilities. 


\subsubsection{Policy advice}

The set of viable regulations (i.e. TACCs) were shown to be restricted below the level that corresponds to MSY, which is noted as an interesting policy consideration given that MSY is widely accepted as a management target and therefore a reference for setting catch limits. This result thus echoes criticisms pointed at the single-species related concept of MSY and the need to consider inter-species dynamics and interrelation of fisheries among other factors when making policy decisions. Similarly, the non-viability of the hard limit, which bases the fish stock's SMBL solely on its biological characteristics (i.e. its carrying capacity) reinforces the inadequacy of the single-species approach.

The Fisheries Act 1996, which governs the management of New Zealand fisheries does have provisions that give scope for consideration of species interactions and externalities when setting or varying sustainability measures. For instance, section 13 of the Fisheries Act 1996 states that the Minister must have "regard to the interdependence of stocks" when setting catch limits. Despite this, in practice controls such as TACCs have frequently been set to achieve single-species MSY-related objectives making New Zealand's QMS essentially a single-species management system (Mace et al., 2013).

One of the concerns expressed in Mace et al. (2013) is how to reconcile the QMS with an ecosystem approach in order to better manage the impacts of fishing on the wider ecosystem. Any ecosystem-based management approach requires considering the complexities and uncertainties of biotic, abiotic, and human components of ecosystems and their interactions and applying an integrated approach to fisheries management within the realms 
of sustainability (FAO, 2003). As demonstrated in this thesis and in other works (e.g. Doyen et al. $(2012,2007)$ ), the viability approach has the potential to provide a practical tool for integrating ecosystem considerations into fisheries management.

Where fisheries are interrelated by e.g. a by-catch production process, it is shown that the actions of economic agents in one fishery can generate negative externalities (here reduced TACCs, which therefore induce lower yields and revenues) on other agents in another fishery, which become potential sources of conflicts among agents. Anticipating the strategic interaction of agents becomes vital for the regulator when determining appropriate TACCs and deemed values. As mentioned earlier, the strategic behaviour (e.g. propensity to deviate from self ACE holding) of an agent in each fishery can be modelled by specifying a differential inclusion for both $\beta_{x}$ and $\beta_{y}^{T}$. In effect, the viability problem becomes a constrained qualitative game (or simply a viability game) and the viability kernel provides an overview for the space in which the game will be played (Krawczyk et al., 2013). This could be useful for a regulator determining the conditions under which cooperation (i.e. $\beta_{x}=\beta_{y}^{T}=1$ ) or non-cooperation (i.e. $\beta_{x} \neq \beta_{y}^{T} \neq 1$ ) can be sustainable. As advocated in Doyen \& Péreau (2012), there is scope for the use of dynamic games and viability theory conjointly to analyse strategic interactions and coordination of users of resources. 


\subsection{Summary}

The by-catch problem is explored in this chapter within the context of New Zealand's QMS. This problem is solved by casting it as a viability problem and then using the numerical application VIKAASA to determine the set of bio-economic configurations delineated by the viability kernel, which guarantee the survival of the fish stock that is primarily caught as by-catch, while maintaining the ecological sustainability of the target fish stock, and fulfilling social and economic requirements.

The viability kernel presents the set of viable regulations (i.e. TACCs) as a function of the target and by-catch biomasses available to the regulator that guarantee the overall system's viability, and therefore constitutes important policy-relevant information for the sustainable management of multi-species fisheries. The non-uniqueness of these choices offers more policy options for negotiations and discussions among different stakeholders than techniques which propose a single optimal policy. Additionally, as demonstrated in Chapter 3, but which is not explicitly shown in the present chapter, nonuniqueness of solutions to the viability problem imply robustness of these solutions given the possibility of parameter and model uncertainties.

The co-viability analysis shows that imposing a non-negative condition on the ACE prices (i.e. economic constraint) induces minimal biomass thresholds that are higher than ecologically acceptable levels as well as ceilings on the TACCs that are lower than the biological maximum. The economic constraint therefore entails more conservative policy choices that reconcile conservation goals with economic requirements. It is also demonstrated that when setting the deemed value properly, this price instrument can be an ef- 
fective tool in constraining catches below TACCs by making it uneconomic to catch beyond self ACE holding.

The potential applications of the co-viability analysis are manifold. It provides a practical tool for a regulator considering integrating the QMS with an EBFM approach. Furthermore, it could also help solve a dynamic game focusing on viability constraints by providing a complete characterisation of its solution.

The next chapter wraps up this thesis by presenting the conclusions and limits drawn from this research. Some discussion is also made as to where the research might be extended. 
128 CHAPTER 4. AN OUTPUT-CONTROLLED FISHERIES MODEL 


\section{Chapter 5}

\section{Conclusion}

This thesis addresses the by-catch fishery management problem (or simply the by-catch problem) in the context of a sustainability problem. Failure to understand the co-dynamics of fish stocks compounded with economic (i.e. profit maximisation) and social (i.e. employment preservation) pressures may threaten the survival of less productive and less valued fish stocks, which are often caught primarily as by-catch. It is claimed that the solution to the sustainability problem is to maintain the system within the realms of safety or acceptability where the conservation of jointly harvested stocks remains compatible with economic and social goals.

It is contended that viability theory provides the right framework for modelling and solving this class of sustainability problems in the sense prescribed in Simon (1955). Rather than maximising an inter-temporal multi-objective function to determine the constrained optimum, the viability approach seeks to attain some sufficient level of a goal variable by analysing the compatibility between the dynamics of a system and its viability constraints, and determining the set of strategies that prevent the system from violating these 
(normative) constraints. Given this, a viability model requires fewer subjectively assessed parameters than the corresponding optimisation model. In particular, no weights are required to materialise the trade-offs between various objectives, and neither is the discount rate needed as the viability approach assigns equal weight to every time period. These features among others render the viability model's solutions less vulnerable to the Lucas (1976) critique and less invasive than those delivered by the optimisation approach (i.e. optimal to exhaust the resource).

Solving the by-catch problem therefore requires casting it as a viability problem and then using an algorithmic method (e.g. VIKAASA) based on viability theory to compute the viability kernel, which delineates the set of viable or sustainable states. A general formulation of a viability problem that is compatible with VIKAASA is presented in Chapter 2. This provides the underlining theoretical framework for the two main viability models developed in this thesis. The goodness of the VIKAASA kernel approximation is verified by reproducing numerically the viability kernel for a calibrated single-species fishery problem that has been solved analytically in Béné et al. (2001).

The first viability model, which is called the "input-controlled fishery model", is developed and solved in Chapter 3. This model is essentially an extension of that proposed in Béné et al. (2001) with the noticeable inclusion of the population dynamics of a by-catch fish stock. A key result for this chapter is the computation of the viability kernel for the input-controlled fishery model. This establishes the configurations required to guarantee the survival of the by-catch stock, while maintaining the ecological sustainability of the target stock and sustaining the fishing activity and profitability 
of the fishery. A comparison of this kernel with that generated under the single-species specification (i.e. Béné et al. (2001)) shows that the size of the former is relatively smaller. More specifically, combinations of relatively high fishing effort and target biomass become non-viable when the co-dynamics of jointly harvested stocks are considered. This finding echoes the criticisms pointed at the single-species approach, which attempts to manage fish stocks independently. Failure to consider the co-dynamics of species in policy decisions may result in certain slower growing stocks being over-fished when exploited at rates appropriate for the target stocks.

The second viability model is called the "output-controlled fisheries model", and is developed and solved in Chapter 4. This model provides a richer platform for policy making, particularly in the context of fisheries management in New Zealand. It builds on the work by Péreau et al. (2012) with the focus on New Zealand's QMS, which basically uses both quantity and price instruments to control fishing output. Although the model abstracts from many of the complexities of both the QMS and the (eco)-system, the viability analysis highlights important policy-relevant considerations. Firstly, the economic constraint induces minimal biomass thresholds that are higher than biologically acceptable levels as well as ceilings on the TACCs that are lower than the biological maximum. The latter implies that MSY is not a viable policy when target stocks are harvested jointly with less productive by-catch stocks. The economic constraint therefore entails more conservative policy choices that reconcile conservation goals with economic requirements. Secondly, these policy choices are generally non-unique and therefore amendable to a regulator's own prioritisation or accommodative to a regulator's willingness to experiment knowing that there are sufficient controls to avert 
a catastrophe. Thirdly, when setting the deemed value properly, it can be an effective tool in constraining catches below the TACCs by making it uneconomic to catch beyond self ACE holding. This price instrument is therefore an effective means of internalising external costs inflicted on other agents.

It is shown that with some minor adjustments to the output-controlled fisheries model, the augmented model can be used to identify the conditions under which cooperation and non-cooperation can be sustainable. However, this would require specifying the viability problem in six dimensions, which is doable but at the cost of significantly increased computational time. The curse of dimensionality problem may be mitigated by running the viability problem on more powerful machines or by employing more efficient kernel detection algorithms that decide where to search based on which points have so far been determined viable. The latter will almost certainly be a focus for future work by the developers of VIKAASA (Krawczyk et al., 2013).

There is increasing interest in shifting management targets from those that maintain fish stocks at levels that maximise production (e.g. MSY) to those that maximise the economic value of the fishery (e.g. MEY) (Kompas et al., 2011; Newell, 2004). The main concern however is how to determine the path(s) to MEY. Given the natural variability of fish stocks and the uncertainties of economic variables (e.g. prices and costs), MEY does not necessarily have to be represented by a single state, but rather a set of states in which a regulator would like to reach at some point in time. This is essentially a finite-time viability problem or a capturability problem, which can be solved through the viability approach by computing the capture basin, which is a subset of the viability kernel $^{1}$.

\footnotetext{
${ }^{1}$ For further discussion on capturability problems, see Section A.1 of Appendix A. At
} 
Another concern raised by New Zealand policy makers in Mace et al. (2013) is that it is not immediately obvious how the QMS can be reconciled with an EBFM approach. It is the aim of this study to show how viability theory can provide policy makers with a better insight of how to integrate ecosystem considerations into the QMS. However, a more comprehensive viability model will have to be developed in order to fully address many of the important issues related to implementing the EBFM approach. This would require building on some of the recent applications (Cissé et al., 2013; Doyen et al., 2013, 2012; Béné \& Doyen, 2008; Doyen et al., 2007) for a more complete account of the ecosystem's structure, processes and functions, as well as the risks ${ }^{2}$ and uncertainties ${ }^{3}$ related to the economic drivers of fisheries. At this stage, VIKAASA is not yet equipped to address issues related to risk, except for the use of differential inclusions to model certain types (tychastic) of uncertainties. However, efforts are underway to incorporate stochastic viability (Doyen \& De Lara, 2010) within VIKAASA.

Moreover, the homogeneity condition on agents will have to be relaxed for a richer analysis of behaviour mechanisms, which are shown in this study to have an effect on the system's viability and therefore the choice of policies (i.e. TACCs). Some of the proposed improvements include allowing for agents to face different unit costs or engage in different strategic behaviours such as

this stage, VIKAASA is not equipped to solve such problems.

${ }^{2}$ These refer to situations where people are capable to formally calculate or intuitively gauge probabilities of losses based on past experience, experimentation, and/or statistical estimation, e.g. investing in a fishing vessel or fish quota.

${ }^{3}$ These refer to situations where quantitative assessment of risk is impossible, e.g. collapse in fish abundance due to unexpected causes or downfall of markets for the fishery's output due to unforseen events. 
contracted, independent and vertically integrated strategic behaviours characterised in Lallemand (2013). Different strategic behaviours should affect agents' propensity to acquire ACE and/or deemed value to cover their catch differently, and therefore have different implications on whether catches are constrained within the TACC. The heterogeneity assumption also allows for an analysis of other important issues such as the rationalisation of the fishing fleet and the distribution of quota, which may entail conflicting economic and social implications.

The parameter values used in this study have been chosen so that the system is consistent with known characteristics of actual multi-species fisheries. They also ensure that both viable and non-viable regions are to be found in the state-space. These serve this study's purpose of presenting a methodology and policy tool for analysing and solving sustainability problems with an emphasis here on a qualitative method. For a real-life quantitative analysis or for the problem's viability kernel to have any quantitative significance, these parameters will have to be re-estimated or re-calibrated using existing biological and economic data ${ }^{4}$. These extensions are left to be addressed in future research.

\footnotetext{
${ }^{4}$ Some discussion on parameter estimation is provided in Appendix B.
} 


\section{Appendix A}

\section{Appendices for Chapter 2}

\section{A.1 A capturability problem}

The viability problem presented in this thesis is mainly concerned with establishing the viability kernel, which formally delineates the conditions by which the evolutionary system always satisfies its environment defined by the set of viability constraints $K$. Alternatively, a regulator may be interested in establishing the conditions by which the system remains viable in $K$ until it reaches a "target" in $K$ in finite time. Such a problem is termed capturability in viability theory and it is formally defined below.

Definition A.1.1 (Capturability). If a subset $C \subset K$ is regarded as a target, an evolution $x(\cdot)$ captures $C$ if there exists a finite time $T$ such that the evolution is viable in $K$ on the interval $[0, T]$ until it reaches the target at $x(T) \in C$ at time $T$.

Associated with the notion of capturability is the concept of viable-capture basin of a target or simply the "capture basin", which is the set of initial con- 
ditions that capture the target in finite time. The capture basin is formally defined below.

Definition A.1.2 (Capture basin). Let $K$ be an environment, $C \in K$ be a target and $F$ an evolutionary system. The capture basin of $C$ (viable in $K$ ) under the evolutionary system $F$ is the $\operatorname{set}^{C_{a p t}}(K, C)$ of initial states $x \in K$ from which starts at least one evolution $x(\cdot) \in F(x)$ viable in $K$ on $[0, T]$ until the finite time $\mathrm{T}$ when the evolution reaches the target at $x(T) \in C$.

The characterisation of the capture basin has a wide range of applications, one of which is to determine the safety configurations required to land an aircraft safely. In that setting, the environment $K$ is represented by the "flight envelop", which describes the boundaries of altitude, the flight path angle and airspeed within which normal flight manoeuvring can be safely conducted. The pilot is tasked with ensuring safety in the last phase of landing by controlling the aircraft such that it remains within the flight envelope until it reaches the "zero altitude" subset of the flight envelope, i.e. the target $C$ at which the aircraft touches down safely. Landing an aircraft safely can thus be formulated as a capturability problem with the objective of delineating the capture basin $\operatorname{Capt}_{F}(K, C)$, which is the set of airspeeds, path angles and altitudes, from which one can reach the runway without ever leaving the flight envelop. This problem is solved in Aubin et al. (2011, p 122). A similar problem is solved in Oishi et al. (2006) where invariance kernels are computed in order to investigate the design of autopilot controls for an aircraft. 


\section{A.2 Gordon-Schaefer model}

Here, selected elements of the Gordon-Schaefer (GS) model are reproduced, with an emphasis on determining the relevant management parameters. A detailed description of the GS model is provided in most fisheries economics textbooks, see e.g. Clark (1990).

\section{A.2.1 Resource dynamics}

Here, an unregulated fishery where a single fish stock harvested by fishers is considered. The natural growth rate of the resource is represented by the notable logistic equation that depends on the intrinsic growth parameter $r>0$ and the environmental carrying capacity $L>0$.

$$
\dot{x}(t)=r x(t)\left(1-\frac{x(t)}{L}\right) \equiv F(x(t)),
$$

which is a second-degree polynomial with two equilibrium solutions, $x \equiv 0$ and $x \equiv L$, graphically shown in Figure A.1a. The fish stock's growth rate is maximised when the stock's biomass is maintained at $x(t)=\frac{L}{2}$, i.e. at half of the stock's carrying capacity.

Using separation of variables, it can be shown that the solution to (A.1) is,

$$
\begin{aligned}
& x(t)=\frac{L}{1+c e^{-r t}}, \\
& \text { where } c=\frac{L-x_{0}}{x_{0}} .
\end{aligned}
$$

One can see that $x(t)$ approaches $L$ at an exponential rate as $t$ tends to infinity, provided that the initial (i.e. $t=0$ ) stock level $x(0)=x_{0}$ is greater 


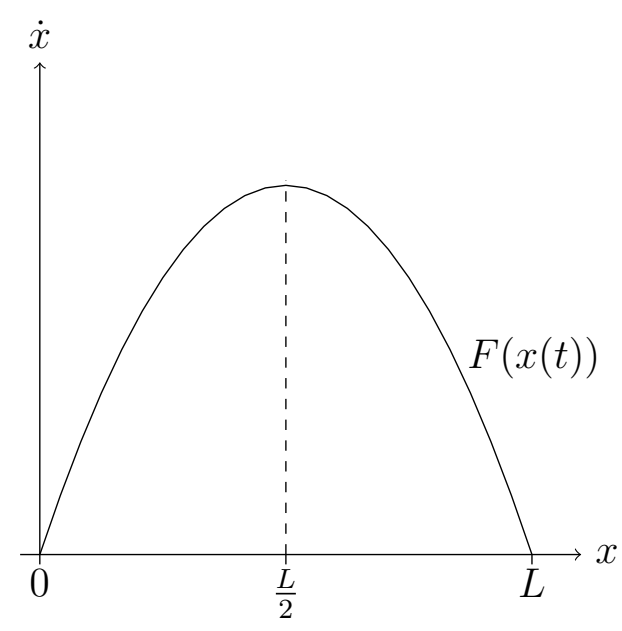

(b)

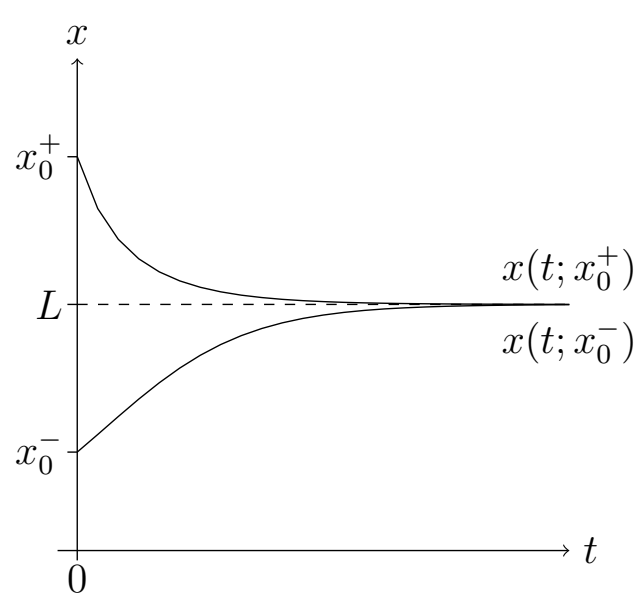

(b)

Figure A.1: (a) The logistic growth rate; (b) Typical solution curves

than zero. Figure A.1b presents the evolution of $x(t)$ for two scenarios, where $x_{0}$ is either above or below the environment's carrying capacity $L$.

Suppose that the renewable resource described by (A.1) is subject to harvest at a rate $H$. It is assumed that harvest is proportional to both the stock's biomass, $x$ and to fishing effort, $e$. The harvest flow is represented by the Graham-Shaefer production function,

$$
H(t)=q e(t) x(t)
$$

where $q$ is a constant parameter usually referred to as the catchability coefficient. The resource dynamic now becomes,

$$
\dot{x}(t)=r x(t)\left(1-\frac{x(t)}{L}\right)-q e(t) x(t) .
$$




\section{A.2.2 Biologically sustainable equilibrium}

Here, the "sustainable" equilibria of (A.4) is determined, under the assumption that $e$ is constant, by setting $\dot{x}=0$ and solving for $x$,

$$
x^{*}=L\left(1-\frac{q e}{r}\right) \text {, }
$$

which is displayed in Figure A.2a. It is observed from (A.5) that $x=0$ if $e=\frac{r}{q}$. This implies that if the level of effort exceeds the critical level $\frac{r}{q}$, the resource stock will be driven towards extinction. However, for any $e<\frac{r}{q}$, there exists a unique non-zero equilibrium defined by (A.5). The equilibrium is sustainable because $\dot{x}=0$ implies that the harvest flow is counterbalanced by stock recruitment, i.e. $H(t)=F(x)$.

By substituting (A.5) into (A.3), Shaefer's "yield-effort curve" is obtained,

$$
Y=q e L\left(1-\frac{q e}{r}\right),
$$

where $Y$ denotes the sustainable yield, $Y=H\left(x^{*}, e\right)$.

Schaefer's yield-effort curve is illustrated in Figure A.2b. For any $e<\frac{r}{q}$, Shaefer's model implies that there is a certain rate of harvest that is just sufficient to capture new additions to the stock of fish, and thus maintains an equilibrium biomass. This rate of harvest, at which $H=F(x)$, is known as the "sustainable yield". With increasing levels of effort, the sustainable yield rises to a maximum level $Y_{\mathrm{MSY}}\left(\right.$ at $e_{\mathrm{MSY}}, x_{\mathrm{MSY}}$ ), and then declines to zero (at $\left.e=\frac{r}{q}, x=0\right)$. It is worth emphasising here that decreases in the sustainable yield for $e>\frac{r}{2 q}$ should not be misconstrued as a prediction that increases in effort beyond this level will result in an immediate reduction in yield. In the short term, (A.3) implies that the catch rate will always increase with effort. However, it is only over the long term, when the processes of resource 


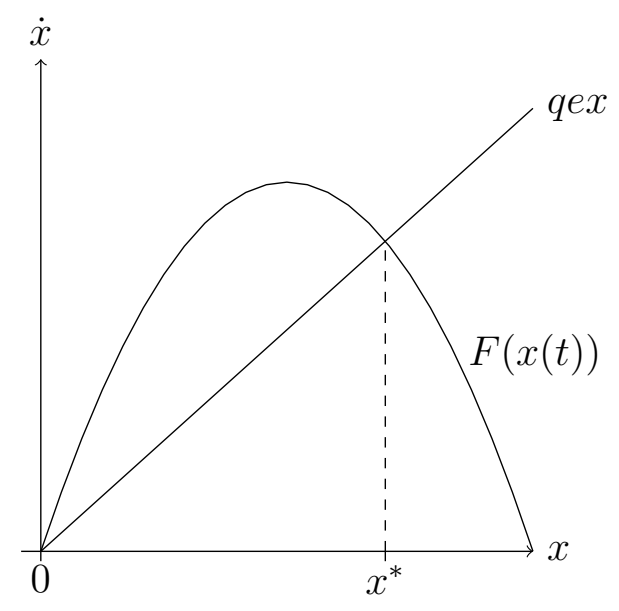

(a)

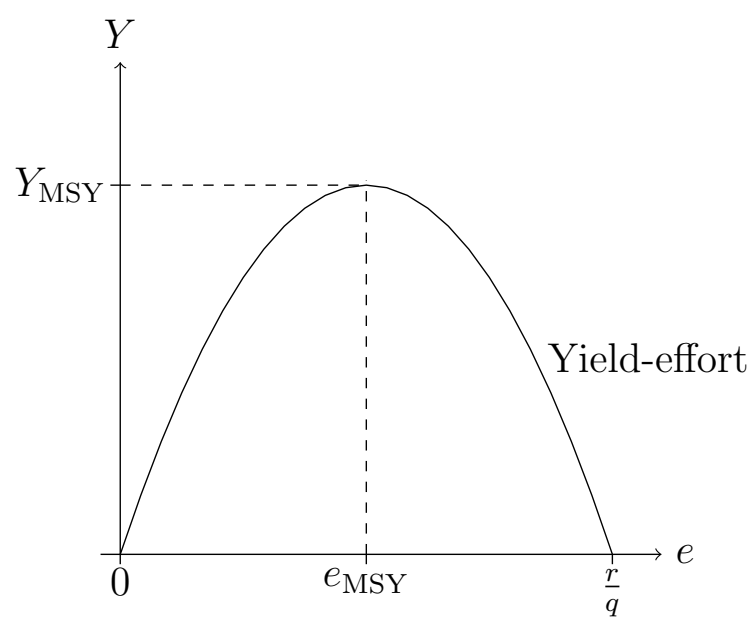

(b)

Figure A.2: (a) The logistic model with constant rate of effort $e$; (b) Shaefer's yield-effort curve

dynamics have resulted in a decreased fish stock, that yield ultimately declines. By the same reasoning, a decrease in $e$ always results in a short-term decrease in the catch rate, but may lead to an increase in the long term.

By setting the first derivative of (A.6) with respect to $e$ to zero and solving for $e$, the level of effort that maximises sustainable yield is obtained,

$$
e_{\mathrm{MSY}}=\frac{r}{2 q}
$$

The maximum sustainable yield, $Y_{\mathrm{MSY}}$ and the corresponding level of stock, $x_{\mathrm{MSY}}$ are obtained by substituting (A.7) into (A.6) and (A.5) respectively,

$$
\begin{aligned}
Y_{\mathrm{MSY}} & =\frac{r L}{4} \\
x_{\mathrm{MSY}} & =\frac{L}{2} .
\end{aligned}
$$


The maximum sustainable yield (MSY) is a pervasive concept in fisheries biology and management. Because there is natural variability over time in both the level and growth of fish stocks, MSY will ideally not be a constant number. The concept also neglects inter-annual environmentally driven stock fluctuations and so is not useful for short term predictions. It is, however, useful for guidance on long term strategy formulation.

\section{A.2.3 Bio-economic equilibrium}

Here, economic aspects of the fishing activity are incorporated through the exogenous fish price $p$ and fishing cost $c$. It is assumed that $p$ is constant and fishers are homogenous. The total cost (TC) of fishing is proportional to the amount of effort applied in the fishery. TC is variable and includes the opportunity cost of fishing. No fixed costs are assumed. Economic profit is therefore represented as,

$$
\pi(t)=p q e(t) x(t)-c e(t)
$$

The "common property or bionomic equilibrium (BE)" stock level $x_{B E}$ is obtained by setting (A.10) to zero and solving for $x$. The corresponding effort level is obtained by substituting (A.11) into (A.5) and solving for $e$,

$$
\begin{aligned}
x_{\mathrm{BE}} & =\frac{c}{p q} \\
e_{\mathrm{BE}} & =\frac{r}{q}\left(1-\frac{c}{p q L}\right) .
\end{aligned}
$$

The bionomic equilibrium stock and effort levels reflect the tragedy of the commons. That is, in an environment where there is an open-access resource, 
economic agents will continue to exploit the resource until economic returns from the resource is dissipated. If $c$ is sufficiently high relative to $p$, i.e. $c>p q L$, the fishery would not be exploited, i.e $e=0$. Additionally, provided $c>0$ the model predicts that exploitation of the fishery would not lead to the extinction of the fish stock.

Figure A.3a shows that $e_{\mathrm{BE}}$ occurs at the point where total sustainable revenue (TSR) equates TC, i.e. sustainable economic profit is zero. Any level of effort above $e_{\mathrm{BE}}$ cannot be maintained indefinitely as this would produce a situation in which economic profit is negative. Some fishers would be forced to withdraw from the fishery and hence reducing the level of effort. For levels of effort below $e_{\mathrm{BE}}$, positive economic profit would attract new entrants and existing fishers to expand effort to capture the extra profit. As long as economic profit is positive, levels of effort will continue to expand until $e_{\mathrm{BE}}$ is reached at which point there is no further incentive to expand. This is the tragedy of the commons.

\section{A.2.4 Maximum economic yield}

For any $e<e_{\mathrm{BE}}$, TSR is above TC. There exists therefore a level of effort $e_{\mathrm{MEY}}$ that maximises total sustainable economic profit. Recall that sustainable yield was defined by (A.6). TSR is the product of the unit fish price $p$ and sustainable yield $Y$ :

$$
\mathrm{TSR}=p q e L\left(1-\frac{q e}{r}\right)
$$

The level of effort that maximises economic profit can be readily obtained by equating the marginal value of effort (MVE) with the marginal cost (MC) of effort and solving for $e$, or as depicted in Figure A.3b, equating the slopes 
of TSR and TC. MVE and MC are simply the slopes of TSR and TC respectively, and can be obtained by differentiating TSR and TC with respect to $e$ respectively,

$$
\begin{aligned}
\mathrm{MVE} & =\frac{\partial \mathrm{TSR}}{\partial e}=p q L\left(1-\frac{2 q e}{r}\right) \\
\mathrm{MC} & =\frac{\partial \mathrm{TC}}{\partial e}=c .
\end{aligned}
$$

Equating MVE to MC and solving for $e$ yields the level of effort that maximises economic profit,

$$
e_{\mathrm{MEY}}=\frac{r}{2 q}\left(1-\frac{c}{p q L}\right)
$$

Note that $e_{M E Y}$ is half the level of effort at the bionomic equilibrium $e_{\mathrm{BE}}$.

To the determine the corresponding levels of biomass and yield when $e_{M E Y}$ is exerted, (A.14) is substituted into (A.5) and (A.6) respectively,

$$
\begin{aligned}
x_{\mathrm{MEY}} & =\frac{L}{2}\left(1+\frac{c}{p q L}\right) \\
Y_{\mathrm{MEY}} & =\frac{r L}{4}\left(1+\frac{c}{p q L}\right)\left(1-\frac{c}{p q L}\right) .
\end{aligned}
$$

Note that as the cost-price ratio $\frac{c}{p}$ tends towards zero, $x_{\mathrm{MEY}}$ and $Y_{\mathrm{MEY}}$ move closer to $x_{\mathrm{MSY}}$ and $Y_{\mathrm{MSY}}$. 
TSR,TC

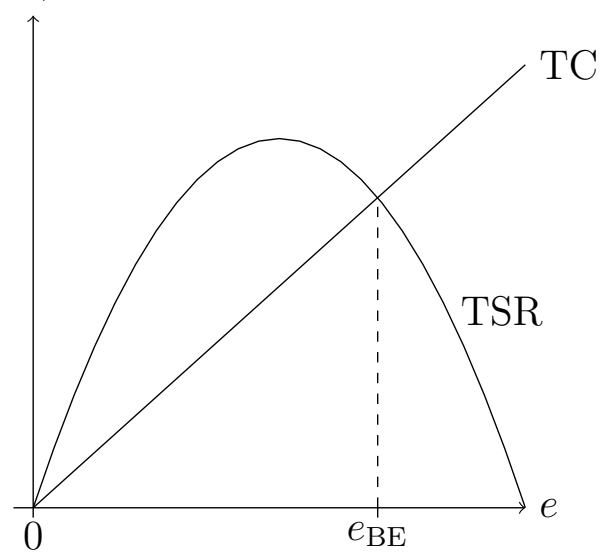

(a)
TSR,TC

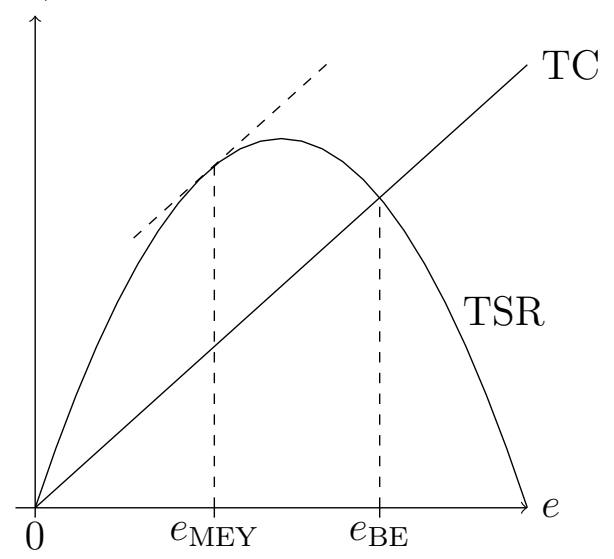

Figure A.3: (a) Bionomic equilibrium level of effort; (b) Maximisation of sustainable economic profit occurs at $e_{\mathrm{MEY}}$

Table A.1: Management parameters for the Gordon-Schaefer model

\begin{tabular}{cccc}
\hline \hline & Yield & Biomass & Effort \\
\hline BE & $\frac{r c}{p q}\left(1-\frac{c}{p q L}\right)$ & $\frac{c}{p q}$ & $\frac{r}{q}\left(1-\frac{c}{p q L}\right)$ \\
MSY & $\frac{r L}{4}$ & $\frac{L}{2}$ & $\frac{r}{2 q}$ \\
MEY & $\frac{r L}{4}\left(1+\frac{c}{p q L}\right)\left(1-\frac{c}{p q L}\right)$ & $\frac{L}{2}\left(1+\frac{c}{p q L}\right)$ & $\frac{r}{2 q}\left(1-\frac{c}{p q L}\right)$ \\
\hline
\end{tabular}




\section{Appendix B}

\section{Appendix for Chapter 3}

\section{B.1 Some brief comments on estimating sur- plus production model parameters}

Biomass dynamic (or surplus production) models, such as that employed in this thesis, are among the most simplest models of fish population dynamics. The major appeal for these models is that they (only) require a time series of both catch and a relative (or absolute) abundance index, e.g. standardised catch-per-unit-effort (CPUE), in order estimate the model's parameters, i.e. $r, q$ and $L$ (Hilborn \& Walters, 1992). Information about the age and size structures of the fish population is generally not required, which is an advantage given the paucity of life-history data of fish stocks prevalent in many developing or low value fisheries ${ }^{1}$. In some cases, biomass dynamic models can provide better estimates of management-related parameters, such as

\footnotetext{
${ }^{1}$ Even some of the richest countries cannot afford to adequately research and assess all stocks captured in their fisheries (Mace et al., 2013).
} 
those presented in Table A.1, than more complex aged-structured models, even when aged-structured data are available (Hilborn \& Walters, 1992).

Fitting surplus production models to observed data should be preferred over dynamic-regression methods as the approach of choice for estimating the parameters (Hilborn \& Walters, 1992). There are several ways of fitting surplus production models. The three most widely used approaches are (1) observation-error estimators (Pella \& Tomlinson, 1969), (2) process-error estimators (Walters \& Hilborn, 1976), and (3) effort-averaging estimators (Fox, 1975). These three approaches have been reviewed and compared in Polacheck et al. (1993). The third approach is a variation of the equilibrium method proposed in Gulland (1961) and assumes that the stock is in equilibrium relative to the recent effort, which is rarely the case in reality and hence one of the reasons why it is rarely used in any formal stock assessment (Polacheck et al., 1993). The observation-error and process-error estimators mainly differ in how errors are introduced in the formal model with parameters to be estimated. The former assumes that all the error occurs in the observed index of abundance whereas the latter assumes that errors only occur in the population dynamics of the stock. Polacheck et al. (1993) show through the use of Monte-Carlo trials that observation-error estimators are the least biased and the most precise, and hence recommended to be used in fitting surplus production models. Given this, the discussion provided henceforth focuses on the observation-error estimator. 
According to Hilborn \& Walters (1992), there are three essential requirements for parameter estimation:

1. A formal model with parameters to be estimated,

$$
x_{t+1}=x_{t}+g\left(x_{t}\right)-H_{t},
$$

where $x_{t}$ is the fishable biomass at the start of year $t$ and $g\left(x_{t}\right)$ is the surplus production, which can take on the Schaefer form $g(x)=$ $r x\left(1-\frac{x}{L}\right)$ as in the case of this thesis ${ }^{2} . H_{t}$ is the harvest rate, which is usually of the Graham-Shaefer form,

$$
H_{t}=q x_{t} e_{t}
$$

where $q$ is the catchability coefficient and $e_{t}$ denotes fishing effort.

An observation model is also required, which relates unobserved stock biomass to an observed abundance index,

$$
O_{t}=q x_{t} e^{\epsilon t}
$$

which essentially follows from (B.2), i.e. $O_{t} \equiv \frac{H_{t}}{e_{t}}$. This is known as CPUE and is widely adopted as a relative index of abundance. The proposition that CPUE is proportional to abundance is questionable (Maunder et al., 2006). Nonetheless, biomass dynamic methods work on any measure of abundance, including absolute abundance surveys, e.g. bottom trawl surveys or hydroacoustics (Hilborn \& Walters, 1992). It is commonly assumed that the error $\epsilon_{t}$ in the observed index satisfy the Gauss-Markov assumptions of independence and identical $N\left(0, \sigma^{2}\right)$ distribution, so the observed index is lognormally distributed about the true abundance. A log-transformation of (B.3) obtains,

$$
\log \left(O_{t}\right)=\log \left(q x_{t}\right)+\epsilon_{t}, \quad \epsilon_{t} \sim N\left(0, \sigma^{2}\right)
$$


2. Data from a population to use to estimate the parameters, which is usually limited to time series of catches and abundance indices.

3. A criterion to judge the goodness of fit to the data of any particular combination of model and parameter estimates. A commonly used criterion is maximum likelihood, which chooses the values of the parameters that maximise the probability that the actual observations would have occurred if the parameters were true.

Thus, the model parameters $r, q, L$ and an additional parameter $x_{\text {initial }}$ (if catch data go back as far as the early years of the fishery, then $x_{\text {initial }}=L$ is assumed), can be estimated by maximising the appropriate likelihood function,

$$
\begin{aligned}
& \mathcal{L}=\prod_{t=1}^{T} \frac{1}{\hat{\sigma}_{t} \sqrt{2 \pi}} e^{-\frac{\hat{\epsilon}_{t}}{2 \hat{\sigma}_{t}^{2}}}, \\
& \text { where } \quad \hat{\epsilon}_{t}=\log \left(O_{t}\right)-\log \left(\hat{O}_{t}\right) \\
& \text { and } \sigma_{t}^{2}=\sum_{t=1}^{T} \frac{\hat{\epsilon}_{t}^{2}}{T} .
\end{aligned}
$$

The product is over all years $(t)$ for which CPUE data are available. $T$ is the total number of CPUE observations. The predicted CPUE is given by $\hat{O}_{t}=\hat{q} \hat{x}_{t}$, where $\hat{x}_{t}$ is obtained by using (B.1) to project the biomass at the start of the catch series $\left(\hat{x}_{\text {initial }}\right)$ forward under the historical annual catches, for a given $\hat{r}$ and $\hat{L}$.

The numerical procedure is basically a search algorithm that (1) begins with inial guesses $\left(\hat{q}, \hat{r}, \hat{L}\right.$ and $\left.\hat{x}_{\text {initial }}\right)$ of the model parameters, (2) projects $\hat{O}_{t}$, and (3) computes the likelihood function (B.5). The algorithm keeps refining $\hat{q}, \hat{r}, \hat{L}$ and $\hat{x}_{\text {initial }}$ until it settles with $q^{*}, r^{*}$, 
$L^{*}$ and $x_{\text {initial }}^{*}$, which maximise (B.5).

Conventional estimation methods such as maximum likelihood tend to be sensitive to the assumption about the model's error structure, so any misspecification of the error structure may result in large errors in parameter estimates when fitting models to data (Chen \& Andrew, 1998). So in any case, the chosen application must be performed with special care. Given this, there is increasing interest to establish more robust estimation methods from which reliable parameter estimates can be attained. For instance, Chen \& Andrew (1998) propose a robust observation-error estimator based on the least median of squares (LMS) in linear regression analyses. Shertzer \& Prager (2002) provide a critique of the LMS based approach and some brief comments on other robust methods such as the method of least absolute values (LAV). For further discussion, see Chen et al. (2003).

One of the problems encountered with abundance indices, in particular CPUE, is that it can be potentially uninformative, e.g. declining catch rate series with little variation in fishing effort (also known as "one way trip" (Hilborn \& Walters, 1992)), making it difficult to clearly estimate productivity and actual abundance simultaneously. The presence of one way trip in CPUE data may explain highly imprecise estimates of $r$ and $L$ using the conventional approaches described earlier (McAllister et al., 2001). This problem can be addressed by estimating $r$ independently.

\footnotetext{
${ }^{2}$ Other forms of $g(x)$ include, $g(x)=r x\left(1-\frac{\log (x)}{\log (L)}\right)$ (Fox form) and $g(x)=$ $\frac{r}{p} x\left(1-\left(\frac{x}{L}\right)^{p}\right)$ (Palla-Tomlinson form).
} 
Using biological information on the life-history of the fish stock, $r$ can be estimated by solving the Euler-Lotka equation (Fisher, 1930),

$$
\sum_{i=0}^{I} e^{(-r i)} w_{i} m_{i} p_{i} \alpha=1,
$$

where $i$ is the age class of the fish stock, $I$ is the oldest age class, $w_{i}$ is the weight-at-age, $m_{i}$ is the maturity-at-age, $p_{i}$ is the survival probability-at-age, and $\alpha$ denotes the recruits-per-spawner biomass.

The weight-at-age $w_{i}$ can be obtained from the weight-length conversion equation,

$$
w_{i}=a l_{i}^{b}
$$

where $a$ and $b$ are the relevant conversion factors, and $l_{i}$ is the length-at-age, which can be obtained from the von Bertalanffy growth equation,

$$
l_{i}=L_{\infty}\left(1-e^{-k\left(i-t_{0}\right)}\right),
$$

where $L_{\infty}, k$ and $t_{0}$ are the von Bertalanffy growth parameters.

The survival probability-at-age $p_{i}$ can be expressed in terms of natural mortality, $M$ :

$$
p_{i}=\prod_{s=0}^{i-1} e^{-M_{s}} .
$$

Mostly all of the relevant life-history parameters for most fish stocks subject to New Zealand QMS can be obtained from the annual fisheries assessment plenary (Ministry for Primary Industries, 2013). Once these parameter values are obtained, the Euler-Lotka equation (B.6) can be solved to estimate the intrinsic growth rate, $r$. For an application of the Euler-Lotka equation to the Indian Ocean bigeye tuna, see FLR Project (2013).

Alternatively, $r$ can be estimated by developing Bayesian prior distributions for $r$ using demographic methods. For further discussion about this 
approach, see McAllister et al. (2001). Once, $r$ is estimated, it is fed into the search algorithm described earlier, which now only estimates $q, L$, and potentially $x_{\text {initial }}$. 


\section{Appendix C}

\section{Appendices for Chapter 4}

\section{C.1 Deriving the ACE price for stock $y$}

Profit for agent $j$ in $F^{T}$ was defined in (4.15). For convenience, it is stated again below,

$$
\pi_{j}=p_{y} h_{j y}-P C_{j}-\Lambda_{j y}-\Omega_{j y}
$$

Using (4.8), (4.11) and (4.13), the long form of the above profit function is obtained,

$$
\begin{aligned}
\pi_{j} & =p_{y} h_{j y}-c_{y} h_{j y}-\frac{w^{T}}{2 y}\left(h_{j y}\right)^{2} \\
& -m_{y} \min \left[h_{j y}, a_{j y}\right]-d_{y} \max \left[0, h_{j y}-a_{j y}\right],
\end{aligned}
$$

which can be re-written as

$$
\begin{aligned}
\pi_{j} & =\left(p_{y}-c_{y}-\frac{w^{T}}{2 y} h_{j y}\right) h_{j y} \\
& -m_{y} \min \left[1, \frac{1}{\beta_{y}^{T}}\right] h_{j y}-d_{y} \max \left[0,1-\frac{1}{\beta_{y}^{T}}\right] h_{j y}
\end{aligned}
$$

by replacing $a_{j y}$ with $\frac{h_{j y}}{\beta_{y}^{T}}$, which is obtained by rearranging (4.2) for $a_{j y}$, and factoring out $h_{j y}$. 
The partial derivative of (C.2) with respect to $h_{j y}$ is

$$
\begin{aligned}
\frac{\partial \pi_{j}}{\partial h_{j y}} & =p_{y}-c_{y}-\frac{w^{T}}{y} h_{j y} \\
& -m_{y} \min \left[1, \frac{1}{\beta_{y}^{T}}\right]-d_{y} \max \left[0,1-\frac{1}{\beta_{y}^{T}}\right]
\end{aligned}
$$

and setting this equal to zero and solving for $h_{j y}$ yields

$$
h_{j y}^{*}=\frac{y}{w^{T}}\left(p_{y}-c_{y}-m_{y} \min \left[1, \frac{1}{\beta_{y}^{T}}\right]-d_{y} \max \left[0,1-\frac{1}{\beta_{y}^{T}}\right]\right) .
$$

Summing up $h_{j y}^{*}$ for all agents in $F^{T}$ yields

$$
\begin{aligned}
H_{y}^{T^{*}} & =\sum_{j=1}^{n^{T}} h_{j y}^{*} \\
& =n^{T} h_{j y}^{*}
\end{aligned}
$$

which is the demand for stock $y$ 's ACE. It is assumed here that the market ACE price for stock $y$ is determined only by the demand for ACE by agents in $F^{T}$, i.e. (C.4) and the supply of ACE, which is the TACC for stock $y$ set by the regulator at the start of the fishing period. Agents in $F^{B}$ purchase whatever ACE is available in the ACE market at the going market price to cover their catch of stock $y$. Thus, the market clearing condition is given by

$$
H_{y}^{T^{*}}=A_{y}
$$

From this, stock $y$ 's market ACE price is deduced

$$
m_{y}^{*}=\max \left[1, \beta_{y}^{T}\right]\left\{p_{y}-c_{y}-d_{y} \max \left[0,1-\frac{1}{\beta_{y}^{T}}\right]-\frac{w^{T}}{y}\left(\frac{A_{y}}{n^{T}}\right)\right\} .
$$




\section{C.2 Supplementary Tables and Graphs}

Table C.1: Surveyed port prices for TAR2

\begin{tabular}{cccccccc}
\hline \hline Fishing year & $2003-04$ & $2004-05$ & $2005-06$ & $2006-07$ & $2007-08$ & $2008-09$ & $2009-10$ \\
\hline \hline Price $(\$ / \mathrm{kg})$ & $\$ 2.42$ & $\$ 2.26$ & $\$ 2.26$ & $\$ 2.31$ & $\$ 3.38$ & $\$ 3.38$ & $\$ 3.38$ \\
\hline
\end{tabular}

Source: Lallemand (2013)

Table C.2: Number of Fishing Permit Holders for TAR2

\begin{tabular}{cccccccc}
\hline \hline Fishing year & $2003-04$ & $2004-05$ & $2005-06$ & $2006-07$ & $2007-08$ & $2008-09$ & $2009-10$ \\
\hline \hline 23 & 23 & 26 & 24 & 26 & 21 & 27
\end{tabular}

Source: Lallemand (2013) 
Table C.3: The base parameter set for the output-controlled fisheries model.

\begin{tabular}{lllll}
\hline \hline Stock-specific & Target stock $(x)$ & \multicolumn{3}{l}{ By-catch stock $(y)$} \\
\hline \hline Description & Symbol & Value & Symbol & Value \\
\hline Intrinsic growth rate & $r_{x}$ & 0.4 & $r_{y}$ & 0.2 \\
Limit carrying capacity (tonnes) & $L_{x}$ & 600 & $L_{y}$ & 300 \\
Price $(\$ / \mathrm{kg})$ & $p_{x}$ & 4 & $p_{y}$ & 2 \\
Linear cost $(\$ / \mathrm{kg})$ & $c_{x}$ & 1.50 & $c_{y}$ & 1 \\
Non-linear cost $(\$)$ & $w^{B}$ & 500 & $w^{T}$ & 250 \\
Deemed value $(\$ / \mathrm{kg})$ & $d_{x}$ & 3 & $d_{y}$ & 1.50 \\
Maximum sustainable yield (tonnes) & $H_{x, \text { MSY }}$ & 60 & $H_{y, \text { MSY }}$ & 15 \\
Minimum guaranteed harvest level (tonnes) & $H_{x, \text { min }}$ & 20 & $H_{y, \text { min }}$ & 5 \\
Maximum TACC adjustment speed & $\delta_{x}$ & 0.01 & $\delta_{y}$ & 0.01 \\
\hline \hline Fishery-specific & By-catch fishery $\left(F^{B}\right)$ & & Target fishery $\left(F^{T}\right)$ \\
\hline \hline Description & Symbol & Value & Symbol & Value \\
\hline By-catch-target harvest ratio & $\alpha$ & 0.2 & - & - \\
Number of agents & $n^{B}$ & 30 & $n^{T}$ & 20 \\
\hline
\end{tabular}




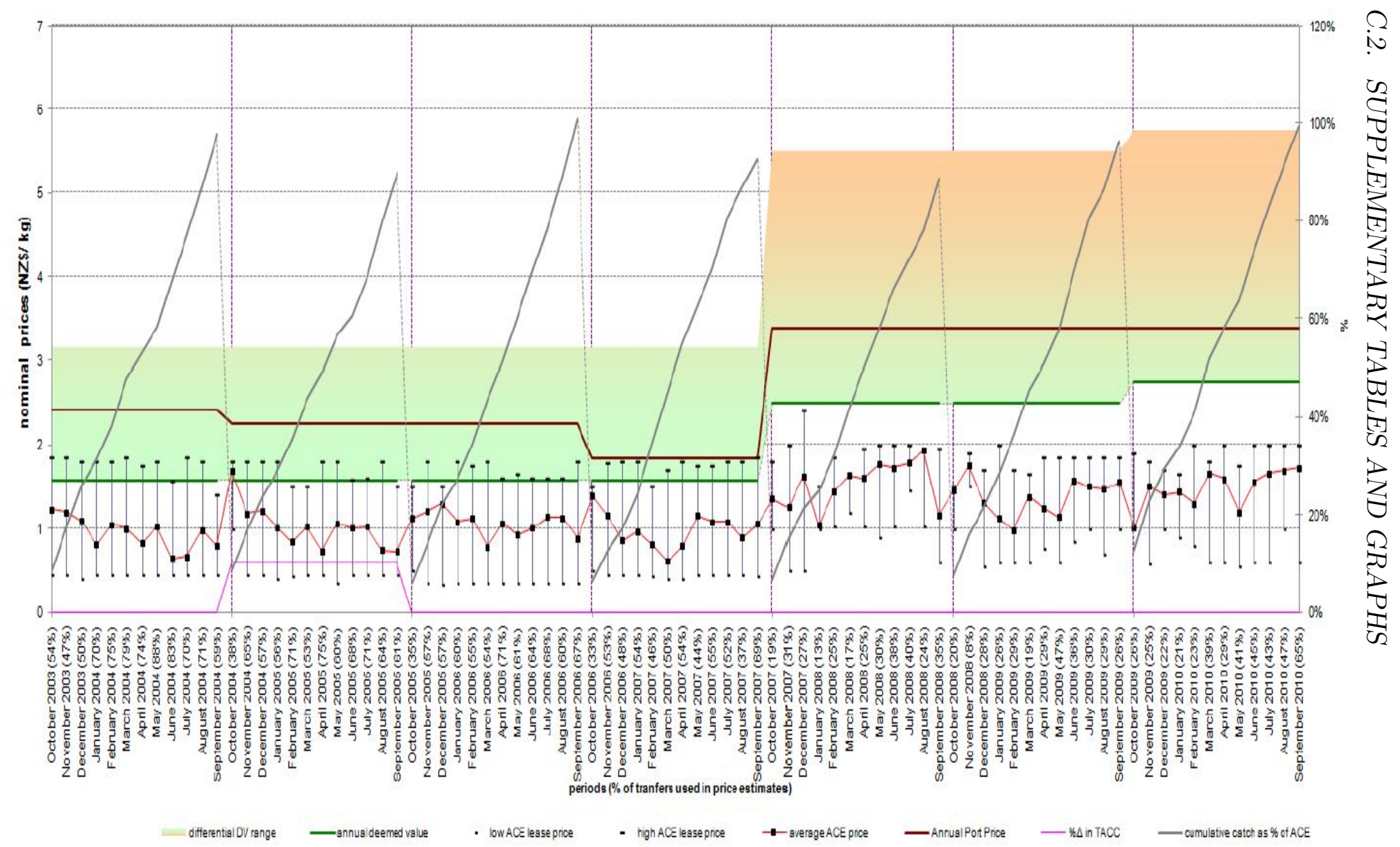

Figure C.1: Monthly trends of high, low, average ACE price, annual deemed value and surveyed port prices for TAR2 (Tarakihi) between fishing years 2003-04 to 2009-10

Source: Lallemand (2013) 


\section{Bibliography}

Abbott, J. \& Wilen, J. (2009). Regulation of fisheries bycatch with commonpool output quotas. Journal of Environmental Economics and Management, 57(2), 195-204.

Alverson, D. L., Freeberg, M. H., Murawski, S. A., \& Pope, J. (1994). A global assessment of fisheries bycatch and discards. Fisheries Technical Paper 339, FAO.

Annala, J. (1996). New Zealand's ITQ system: have the first eight years been a success or a failure? Reviews in Fish Biology and Fisheries, 6(1), $43-62$.

Arkema, K. K., Abramson, S. C., \& Dewsbury, B. M. (2006). Marine ecosystem-based management: from characterization to implementation. Frontiers in Ecology and the Environment, 4 (10), 525-532.

Aubin, J. (1991). Viability theory. Boston, MA: Birkhäuser.

Aubin, J., Pujal, D., \& Saint-Pierre, P. (2005). Dynamic Management of Portfolios with Transaction Costs under Tychastic Uncertainty. In $\mathrm{Nu}$ merical Methods in Finance. New York, NY: Springer US. 
Aubin, J.-P. (1990). A survey of viability theory. SIAM Journal on Control and Optimization, 28(4), 749-788.

Aubin, J. P., Bayen, A. M., \& Saint-Pierre, P. (2011). Viability theory: new directions. Berlin, Germany: Springer-Verlag.

Begg, D., Fisher, S., \& Dornbusch, R. (2003). Economics (7 ed.). Berkshire, UK: McGraw-Hill Education.

Béné, C. \& Doyen, L. (2008). Contribution values of biodiversity to ecosystem performances: A viability perspective. Ecological Economics, 68(1), 14-23.

Béné, C., Doyen, L., \& Gabay, D. (2001). A viability analysis for a bioeconomic model. Ecological Economics, 36(3), 385-396.

Bentley, N. (2010). Characterisation of FMA 2 fisheries: species summaries. Technical report, Ministry for Primary Industries.

Beverton, R. \& Holt, S. (1957). On the dynamics of exploited fish populations. Fisheries Investigations Series 2(19). London, UK: Ministry of Agriculture, Fisheries and Food.

Bonneuil, N. (2000). Viability in dynamic social networks. Journal of Mathematical Sociology, 24(3), 175-192.

Bonneuil, N. (2003). Making ecosystem models viable. Bulletin of Mathematical Biology, 65 (6), 1081-1094.

Bonneuil, N. \& Saint-Pierre, P. (2008). Beyond Optimality: Managing Children, Assets, and Consumption over the Life Cycle. Journal of Mathematical Economics, 44(3-4), 227-241. 
Braun, D. R., Harris, J. W., Levin, N. E., McCoy, J. T., Herries, A. I., Bamford, M. K., Bishop, L. C., Richmond, B. G., \& Kibunjia, M. (2010). Early hominin diet included diverse terrestrial and aquatic animals $1.95 \mathrm{Ma}$ in East Turkana, Kenya. Proceedings of the National Academy of Sciences, $107(22), 10002-10007$.

Chapel, L., Deffuant, G., Martin, S., \& Mullon, C. (2008). Defining yield policies in a viability approach. Ecological Modelling, 212(1), 10-15.

Charles, A. T. (1994). Towards sustainability: the fishery experience. Ecological Economics, $11(3), 201-211$.

Chen, Y. \& Andrew, N. (1998). Parameter estimation in modelling the dynamics of fish stock biomass: are currently used observation-error estimators reliable? Canadian Journal of Fisheries and Aquatic Sciences, $55(3), 749-760$.

Chen, Y., Jiao, Y., \& Chen, L. (2003). Developing robust frequentist and Bayesian fish stock assessment methods. Fish and Fisheries, 4 (2), 105-120.

Cheung, W. W. \& Sumaila, U. R. (2008). Trade-offs between conservation and socio-economic objectives in managing a tropical marine ecosystem. Ecological Economics, 66(1), 193-210.

Cissé, A., Gourguet, S., Doyen, L., Blanchard, F., \& Péreau, J.-C. (2013). A bio-economic model for the ecosystem-based management of the coastal fishery in French Guiana. Environment and Development Economics, 18(3), 245-269. 
Clark, C. (1990). Mathematical bioeconomics. New York, NY: John Wiley and Sons.

Cury, P., Mullon, C., Garcia, S., \& Shannon, L. (2005). Viability theory for an ecosystem approach to fisheries. ICES Journal of Marine Science, 62(3), 577-584.

De Lara, M., Doyen, L., Guilbaud, T., \& Rochet, M. (2007). Is a management framework based on spawning-stock biomass indicators sustainable? A viability approach. ICES Journal of Marine Science, 64(4), 761-767.

De Lara, M. \& Martinet, V. (2009). Multi-criteria dynamic decision under uncertainty: A stochastic viability analysis and an application to sustainable fishery management. Mathematical Biosciences, 217(2), 118-124.

De Lara, M., Ocana, E., Oliveros-Ramos, R., \& Tam, J. (2012). Ecosystem viable yields. Environmental Modeling \& Assessment, 17(6), 565-575.

Deffuant, G., Chapel, L., \& Martin, S. (2007). Approximating viability kernels with support vector machines. IEEE Transactions on Automatic Control, 52(5), 933-937.

Doll, J. (1988). Traditional economic models of fishing vessels: a review with discussion. Marine Resource Economics, 5(2), 99-123.

Doyen, L., Cissé, A., Gourguet, S., Mouysset, L., Hardy, P.-Y., Béné, C., Blanchard, F., Jiguet, F., Péreau, J.-C., \& Thébaud, O. (2013). Ecologicaleconomic modelling for the sustainable management of biodiversity. Computational Management Science, 10(4), 353-364. 
Doyen, L. \& De Lara, M. (2010). Stochastic viability and dynamic programming. Systems \& Control Letters, 59(10), 629-634.

Doyen, L., De Lara, M., Ferraris, J., \& Pelletier, D. (2007). Sustainability of exploited marine ecosystems through protected areas: a viability model and a coral reef case study. Ecological Modelling, 208(2), 353-366.

Doyen, L. \& Péreau, J.-C. (2012). Sustainable coalitions in the commons. Mathematical Social Sciences, 63(1), 57-64.

Doyen, L. \& Saint-Pierre, P. (1997). Scale of viability and minimal time of crisis. Set-Valued Analysis, 5(3), 227-246.

Doyen, L., Thébaud, O., Béné, C., Martinet, V., Gourguet, S., Bertignac, M., Fifas, S., \& Blanchard, F. (2012). A stochastic viability approach to ecosystem-based fisheries management. Ecological Economics, 75, 32-42.

Eisenack, K., Scheffran, J., \& Kropp, J. P. (2006). Viability analysis of management frameworks for fisheries. Environmental Modeling $\&$ Assessment, $11(1), 69-79$.

FAO (2003). Fisheries management. The ecosystem approach to fisheries. Technical Guidelines for Responsible Fisheries 4 (Suppl. 2), FAO.

FAO (2012). The state of world fisheries and aquaculture 2012. Rome, Italy: Author.

Fisher, R. (1930). The Genetical Theory of Natural Selection. Oxford, UK: Oxford University Press. 
Fletcher, W. (2005). The application of qualitative risk assessment methodology to prioritize issues for fisheries management. ICES Journal of Marine Science, 62(8), 1576-1587.

FLR Project (2013). Assessment using production models. Retrieved from http://flr-project.org/doku.php?id=tutorials: spmodels.

Fox, W. W. (1975). Fitting the generalized stock production model by leastsquares and equilibrium approximation. Fishery Bulletin, 73(1), 23-37.

Frankowska, H. \& Quincampoix, M. (1990). Viability kernels of differential inclusions with constraints: algorithms and applications. Working Paper 90-64, International Institute for Applied Systems Analysis.

Gaitsgory, V. \& Quincampoix, M. (2009). Linear programming approach to deterministic infinite horizon optimal control problems with discounting. SIAM Journal on Control and Optimization, 48(4), 2480-2512.

Gordon, H. S. (1954). The economic theory of a common-property resource: the fishery. The Journal of Political Economy, 62(2), 124-142.

Gulland, J. A. (1961). Fishing and the stocks of fish at Iceland. Fishery investigations (series 2), 23(4), 1-52.

Guyader, O. \& Thébaud, O. (2001). Distributional issues in the operation of rights-based fisheries management systems. Marine Policy, 25(2), 103112.

Hammond, T. \& Trenkel, V. (2005). Censored catch data in fisheries stock assessment. ICES Journal of Marine Science, 62(6), 1118-1130. 
Hauge, K. H., Cleeland, B., \& Wilson, D. C. (2009). Fisheries depletion and collapse. Lausanne, Switzerland: International Risk Governance and Council.

Herrera, G. (2005). Stochastic bycatch, informational asymmetry, and discarding. Journal of Environmental Economics and Management, 49(3), $463-483$.

Hilborn, R. (2007). Defining success in fisheries and conflicts in objectives. Marine Policy, 31(2), 153-158.

Hilborn, R. \& Walters, C. J. (1992). Quantitative Fisheries Stock Assessment: choice, dynamics and uncertainty. New York, NY: Chapman \& Hall.

Holland, D. S. \& Herrera, G. E. (2006). Flexible catch balancing policies for multispecies individual fishery quotas. Canadian Journal of Fisheries and Aquatic Sciences, 63(8), 1669-1684.

Hotelling, H. (1931). The economics of exhaustible resources. The Journal of Political Economy, 39(2), 137-175.

Kelleher, K. (2005). Discards in the world's marine fisheries: an update. Fisheries Technical Paper 470, FAO.

Kompas, T., Grafton, R. Q., \& Che, N. (2011). Target and path: maximum economic yield in fisheries management. Technical Report 11.3, Austrlian Bureau of Agricultural and Resource Economics and Sciences.

Krawczyk, J. \& Kim, K. (2009). Satisfying Solutions to a Monetary Policy Problem. Macroeconomics Dynamics, 13(1), 46-80. 
Krawczyk, J., Pharo, A., Serea, O. S., \& Sinclair, S. D. (2013). Computation of viability kernels: a case study of by-catch fisheries. Computational Management Science, 10(4), 365-396.

Krawczyk, J. B. \& Kim, K. (2013). Viable Stabilising Non-Taylor Monetary Policies for an Open Economy. Computational Economics, Online First.

Krawczyk, J. B. \& Pharo, A. (2013). Viability theory: an applied mathematics tool for achieving dynamic systems' sustainability. Mathematica Applicanda, 41(1), 97-126.

Krawczyk, J. B. \& Pharo, A. S. (2011). Manual of VIKAASA: An Application Capable of Computing and Graphing Viability Kernels for Simple Viability Problems. SEF Working Paper 13/2011, Victoria University of Wellington.

Krawczyk, J. B. \& Serea, O. S. (2011). When can it be not optimal to adopt a new technology? A viability theory solution to a two-stage optimal control problem of new technology adoption. Optimal Control Applications and Methods, $34(2), 127-144$.

Krawczyk, J. B., Sissons, C., \& Vincent, D. (2012). Optimal versus satisfactory decision making: a case study of sales with a target. Computational Management Science, 9(2), 233-254.

Lallemand, P. D. (2013). Incorporating Economics into Traditional CPUE Analyses. New Zealand Fisheries Assessment Report 2013/61, Ministry of Primary Industries.

Little, L., Punt, A., Mapstone, B., Begg, G., Goldman, B., \& Williams, 
A. (2009). An agent-based model for simulating trading of multi-species fisheries quota. Ecological Modelling, 220(23), 3404-3412.

Lock, K. \& Leslie, S. (2007). New Zealand's Quota Management System: A history of the first 20 years. Working Paper 07-02, Motu Economic and Public Policy Research.

Lucas, R. J. (1976). Econometric policy evaluation: A critique. CarnegieRochester Conference Series on Public Policy, 1(1), 19-46.

Mace, P. M., Sullivan, K. J., \& Cryer, M. (2013). The evolution of New Zealand's fisheries science and management systems under ITQs. ICES Journal of Marine Science, Online First.

Marchal, P., Little, L., \& Thébaud, O. (2011). Quota allocation in mixed fisheries: a bioeconomic modelling approach applied to the Channel flatfish fisheries. ICES Journal of Marine Science, 68(7), 1580-1591.

Martinet, V. \& Doyen, L. (2007). Sustainability of an economy with an exhaustible resource: A viable control approach. Resource and energy economics, 29(1), 17-39.

Martinet, V., Thébaud, O., \& Doyen, L. (2007). Defining viable recovery paths toward sustainable fisheries. Ecological Economics, 64(2), 411-422.

Martinet, V., Thébaud, O., \& Rapaport, A. (2010). Hare or Tortoise? Tradeoffs in Recovering Sustainable Bioeconomic Systems. Environmental Modeling and Assessment, 15(6), 503-517.

Maunder, M. N., Sibert, J. R., Fonteneau, A., Hampton, J., Kleiber, P., \& Harley, S. J. (2006). Interpreting catch per unit effort data to assess 
the status of individual stocks and communities. ICES Journal of Marine Science, 63(8), 1373-1385.

McAllister, M., Pikitch, E. K., \& Babcock, E. (2001). Using demographic methods to construct Bayesian priors for the intrinsic rate of increase in the Schaefer model and implications for stock rebuilding. Canadian Journal of Fisheries and Aquatic Sciences, 58(9), 1871-1890.

Metcalf, S., Gaughan, D. J., \& Shaw, J. (2009). Conceptual models for ecosystem based fisheries management (EBFM) in Western Australia. Fisheries Research Report 194, Department of Fisheries.

Ministry for Primary Industries (2005). Fisheries Management in New Zealand. Retrieved from http://www.fish.govt.nz/en-nz/ Publications/Ministerial+Briefings/Ministerial+Briefing+05/ Annex+1+-+Fisheries+Management+in+New+Zealand/Fisheries+ management+in+New+Zealand.htm.

Ministry for Primary Industries (2013). Fisheries Assessment Plenary, May 2013: stock assessments and yield estimates. Wellington, New Zealand: Author.

Ministry of Fisheries (2008). Harvest Strategy Standard for New Zealand. Wellington, New Zealand: Author.

Mullon, C., Cury, P., \& Shannon, L. (2004). Viability model of trophic interactions in marine ecosystems. Natural Resource Modeling, 17(1), 71102. 
Newell, R. (2004). Maximising value in multi-species fisheries. Fullbright New Zealand. Retrieved from http://www.fulbright.org.nz/wp-content/ uploads/2011/12/axford2004_newell.pdf.

Oishi, M., Mitchell, I., Tomlin, C., \& Saint-Pierre, P. (2006). Computing viable sets and reachable sets to design feedback linearizing control laws under saturation. In Proceedinds of the 45th IEEE Conference on Decision and Control, (pp. 3801-3807).

Pascoe, S. (1997). Bycatch management and the economics of discarding. Fisheries Technical Paper 370, FAO.

Pascoe, S., Innes, J., Holland, D., Fina, M., Thébaud, O., Townsend, R., Sanchirico, J., Arnason, R., Wilcox, C., \& Hutton, T. (2010). Use of incentive-based management systems to limit bycatch and discarding. International Review of Environmental and Resource Economics, 4(2), 123161.

Pauly, D., Christensen, V., Guénette, S., Pitcher, T. J., Sumaila, U. R., Walters, C. J., Watson, R., \& Zeller, D. (2002). Towards sustainability in world fisheries. Nature, 418(6898), 689-695.

Peacey, J. (2002). Managing Catch Limits in Multi-Species, ITQ Fisheries. In Proceedings of the 11th International Institute of Fisheries Economics and Trade. Retrieved from http://www.fish.govt.nz/en-nz/Archive/ News/Press+Releases+2003/March+2003/IIFET+Conference+Paper+ Managing+Catch+Limits+in+Multi-Species+ITQ+Fisheries.htm.

Pella, J. J. \& Tomlinson, P. K. (1969). A generalized stock production model. Bulletin of the Inter-American Tropical Tuna Commission, 13, 416-496. 
Péreau, J.-C., Doyen, L., Little, L., \& Thébaud, O. (2012). The triple bottom line: Meeting ecological, economic and social goals with individual transferable quotas. Journal of Environmental Economics and Management, 63(3), 419-434.

Polacheck, T., Hilborn, R., \& Punt, A. (1993). Fitting surplus production models: comparing methods and measuring uncertainty. Canadian Journal of Fisheries and Aquatic Sciences, 50(12), 2597-2607.

Pope, J. G. (2002). Input and output controls: the practice of fishing effort and catch management in responsible fisheries. In K. L. Cochrane (Ed.), A fishery manager's guidebook. Management measures and their application. (pp. 75-94). Rome, Italy: FAO.

Saint-Pierre, P. (1994). Approximation of the viability kernel. Applied Mathematics and Optimization, 29(2), 187-209.

Sanchirico, J., Holland, D., Quigley, K., \& Fina, M. (2006). Catch-quota balancing in multispecies individual fishing quotas. Marine Policy, 30(6), $767-785$.

Schaefer, M. (1954). Some aspects of the dynamics of populations important to the management of the commercial marine fisheries. Bulletin of the Inter-American Tropical Tuna Commission, 2(1), 26-56.

Shertzer, K. W. \& Prager, M. H. (2002). Least median of squares: a suitable objective function for stock assessment models? Canadian Journal of Fisheries and Aquatic Sciences, 59(9), 1474-1481. 
Simon, H. (1955). A behavioral model of rational choice. The quarterly journal of economics, 69(1), 99-118.

Squires, D., Campbell, H., Cunningham, S., Dewees, C., Grafton, R., Herrick, S., Kirkley, J., Pascoe, S., Salvanes, K., Shallard, B., et al. (1998). Individual transferable quotas in multispecies fisheries. Marine Policy, 22(2), 135-159.

Stewart, J. \& Callagher, P. (2011). Quota concentration in the New Zealand fishery: annual catch entitlement and the small fisher. Marine Policy, 35(5), 631-646.

Sumaila, U. (2010). A cautionary note on individual transferable quotas. Ecology and Society, 15(3), 36.

Tahvonen, O. (2010). Age-structured optimization models in fisheries bioeconomics: a survey. In R. Boucekkine, N. Hritonenko, \& Y. Yatsenko (Eds.), Optimal Control of Age-structured Populations in Economy, Demography, and the Environment (pp. 140-173). New York, NY: Routledge.

Townsend, R. (1986). A critique of models of the American lobster fishery. Journal of Environmental Economics and Management, 13(3), 277-291.

van Zyl, B. (2012). No fish today. Retrieved from http://www youtube. $\mathrm{com} /$ watch? $\mathrm{v}=\mathrm{u} 6$ Wt2N7bhJQ\&f eature=share.

Wachsman, Y. (2003). Externalities and Management Regimes in Fisheries Exploitation. JIMAR Contribution 02-346, Joint Institute for Marine and Atmospheric Research. Retrieved from http://www.soest.hawaii.edu/ pfrp/soest_jimar_rpts/wachsman03.pdf. 
Walker, S. \& Townsend, R. (2008). Economic analysis of New Zealand's deemed value system. In Proceedings of the 14 th International Institute of Fisheries Economics and Trade. Retrieved from http://ir.library.oregonstate.edu/xmlui/bitstream/handle/ 1957/37722/199.pdf? sequence=1.

Walters, C. J. \& Hilborn, R. (1976). Adaptive control of fishing systems. Journal of the Fisheries Board of Canada, 33(1), 145-159.

WCED (1987). Our Common Fiuture. Oxford, UK: Oxford University Press.

Wilen, J. (2000). Renewable resource economists and policy: what differences have we made? Journal of Environmental Economics and Management, $39(3), 306-327$. 


\section{Glossary}

ACE Annual catch entitlement is the catching right (denominated in tonnes) generated each year from quota.

deemed value $\mathrm{A}$ fee $(\$ / \mathrm{kg})$ incurred for catches in excess of $\mathrm{ACE}$.

DVS Deemed value system.

EBFM Ecosystem-based fishery management.

FMAs Fisheries management areas.

hard limit Reference point $\left(10 \% B_{0}\right)$ below which stocks are deemed to be collapsed.

ITQ Individual transferable quota is the in-perpetuity right to receive a share of the TACC each year.

MEY Maximum economic yield.

MGHL Minimum guaranteed harvest level.

MSY Maximum sustainable yield. 
QMA Quota Management Area.

QMS Quota Management System.

SMBL Safe minimum biomass level.

TAC Total allowable catch is the total regulated catch for a fish stock in a fishing year.

TACC Total allowable commercial catch is the total regulated commercial catch for a fish stock in a fishing year.

TAR2 Tarakihi (Nemadactylus macropterus) in QMA2.

VIKAASA Viability Kernel Approximation Analysis and Simulation Application is an application that uses numerical methods to approximate and visualise viability kernels. 


\section{List of Symbols}

$\alpha$ The by-catch-target harvest ratio.

$a_{i x}(t)$ Agent $i$ 's ACE holding for stock $x$ after trade in period $t$.

$a_{i y}(t)$ Agent $i$ 's ACE holding for stock $y$ after trade in period $t$.

$a_{j y}(t)$ Agent $j$ 's ACE holding for stock $y$ after trade in period $t$.

$A_{x}(t)$ Fish stock $x$ 's TACC.

$A_{y}(t)$ Fish stock $y$ 's TACC.

$\beta_{y}^{B}$ An indicator of whether (on average) $F^{B}$ agents' catch of stock $y$ differs from their ACE holding for that stock and in which direction.

$\beta_{y}^{T}$ An indicator of whether (on average) $F^{T}$ agents' catch of stock $y$ differs from their ACE holding for that stock and in which direction.

$\beta_{x}$ An indicator of whether (on average) $F^{B}$ agents' catch of stock $x$ differs from their ACE holding for that stock and in which direction.

$C$ Fixed cost.

$c$ Marginal cost of effort. 
$c_{x}$ Unit cost independently attributed to harvesting stock $x$.

$c_{y}$ Unit cost independently attributed to harvesting stock $y$.

$d_{x}$ The deemed value of stock $x$.

$d_{y}$ The deemed value of stock $y$.

$e(t)$ Fishing effort.

$F^{B}$ By-catch fishery in which fish stocks $x$ and $y$ are jointly harvested.

$F^{T}$ Target fishery in which only fish stock $y$ is harvested.

$h_{i x}(t)$ Agent $i$ 's harvest of stock $x$.

$h_{i y}(t)$ Agent $i$ 's harvest of stock $y$.

$h_{j y}(t)$ Agent $j$ 's harvest of stock $y$.

$H_{x}(t)$ Total harvests of fish stock $x$.

$H_{y}(t)$ Total harvests of fish stock $y$.

$i$ An individual agent in the by-catch fishery.

$j$ An individual agent in the target fishery.

$K$ The viability constraint set.

$L_{x}$ Limit carrying capacity for fish stock $x$.

$L_{y}$ Limit carrying capacity for fish stock $y$. 
$m_{x}(t)$ The ACE price of stock $x$.

$m_{y}(t)$ The ACE price of stock $y$.

$n^{B}$ The number of active agents in the by-catch fishery.

$n^{T}$ The number of active agents in the target fishery.

$p_{x}$ Unit price of fish stock $x$.

$p_{y}$ Unit price of fish stock $y$.

$q_{x}$ Catchability coefficient of fish stock $x$.

$r_{x}$ Intrinsic growth rate for fish stock $x$.

$r_{y}$ Intrinsic growth rate for fish stock $y$.

$t$ Time period and it usually refers to the fishing year.

$\Theta$ Time horizon - an infinite time horizon is assumed in this thesis.

$\mathcal{V}$ The viability kernel.

$w^{B}$ Cost associated with the non-linear component of agent $i$ production cost function.

$w^{T}$ Cost associated with the non-linear component of agent $j$ ' production cost function.

$x(t)$ Fish stock $x$ 's fishable biomass.

$y(t)$ Fish stock $y$ 's fishable biomass. 